\title{
Abstracts for the Genes for Health Meeting, incorporating the Inaugural GRaPH-Int Conference and the 33rd HGSA Annual Scientific Meeting
}

\author{
Fremantle, Australia, May 3-6, 2009
}

\begin{abstract}
TRANSCRIPTOME PATTERNS SHOW NORMALIZATION OF MUSCLE GENE EXPRESSION AFTER RESTORATION OF DYSTROPHIN IN MDX MICE BY EXON SKIPPING [146]

A.Adams, S. Fletcher, T. Ly, R. Johnsen, and S.Wilton

Molecular Genetic Therapy Group, CNND, University of Western Australia, Perth, Australia

Duchenne muscular dystrophy (DMD) is a recessive X-linked form of muscular dystrophy caused by protein-truncating mutations in the dystrophin gene. It is characterized by rapid progression of muscle degeneration, eventually leading to loss of ambulation and death from respiratory or cardiac complications. The $m d x$ mouse model of muscular dystrophy has a nonsense mutation in exon 23 of the dystrophin gene. We have previously shown that a morpholino antisense oligomer can induce removal of this exon, and result in an in-frame mRNA transcript encoding a shortened but functional dystrophin protein. In-order to further investigate the molecular processes associated with dystrophin restoration, we used Genechip Exon Arrays to compare expression levels in the diaphragm of 10 week-old treated $m d x$ mice to that of age-matched $m d x$ and C57B1/10 control mice. Quality control and statistical analysis were necessary to normalise microarray data. A one-way ANOVA and fold changes were used to compile gene lists. These genes were categorized based on gene ontology classification; for example, signal transduction, ion transport etc, and then analysed in more detail. The data arising from this study should provide a greater understanding about gene interactions and their affects on pathways that are involved in muscle regeneration, and may contribute to the development of new therapies for DMD.
\end{abstract}

\section{ESTABLISHING A MULTIDISCIPLINARY}

HAEMOGLOBINOPATHIES CLINIC IN WESTERN

AUSTRALIA: THE GENETIC COUNSELLOR [119]

\section{S.Ager}

Genetic Services of Western Australia, King Edward Memorial Hospital, Subiaco, Western Australia, Australic

Hemoglobinopathies are a group of inherited blood disorders that may be associated with significant morbidity and mortality. They are known to occur more commonly in people of particular geographic origins, including Asia, Africa, the Middle East and the Mediterranean. As a result of immigration, couples with, or at risk of having, affected children are being seen more frequently at King Edward Memorial Hospital and Princess Margaret Hospital. To date their management has often been uncoordinated, which may result in overuse of healthcare resources and suboptimal patient care and education. A multidisciplinary hemoglobinopathies clinic is currently being established in Western Australia, with the aim of providing coordinated and optimal care to families affected by, or at risk of, this group of conditions. The healthcare providers that will be involved with the clinic are from a range of specialty backgrounds, including genetics, hematology, obstetrics, neonatology, pediatrics, nursing, pathology, migrant health and population health. The clinic will be unique within Western Australia and its establishment is likely to be both rewarding and challenging. As the genetic counselor associated with the clinic, I present my experiences to date, which may be useful to other genetic counselors who are involved in specialized, multidisciplinary clinics in the future.

\section{EDUCATIONAL RESOURCES FOR PUBLIC HEALTH}

\section{GENOMICS [53]}

\section{C.Alberg, P. Brice, and H. Burton}

Foundation for Genomics and Population Health (PHG Foundation), United Kingdom

A suite of ten free animated tutorials in key areas relating to public health genomics are available from the PHG Foundation website. These tutorials, which are aimed primarily at health professionals but are widely accessible, can be used individually or as stand-alone modules, and are available under a Creative Commons license. Topics covered are:
- DNA Test Methods and Technologies — from DNA sequencing to microarrays.

- Twin Adoption Studies - using epidemiological tools to examine the degree of genetic influence in different diseases.

- Disease Susceptibility - using linkage and gene-disease association studies to identify genetic predisposition towards complex diseases.

- The ACCE Framework for Genetic Test Evaluation - considering analytical validity, clinical validity and utility, and ethical, legal and social issues.

- Clinical Utility - colorectal cancer is used as an exemplar for considering components of clinical utility in greater detail.

- Penetrance - explaining the concept of penetrance in inherited disease with examples.

- Patents - including the patent filing process and what can and cannot be patented.

- Moral Theories - consequentialism, deontology and bioethics, with scenarios.

- Pharmacogenomics - current understanding of genetic influences on drug response and examples of how this knowledge may be used for personalised medicine.

- Informed Consent - an overview of different levels of consent and why it is necessary.

\section{PROGRESS IN DEVELOPMENT OF A NATIONAL CARDIAC GENETICS SERVICE IN THE LIGHT OF RAPID SCIENTIFIC} ADVANCES [34]

\section{C.Alberg, H. Burton, and A. Stewart}

Foundation for Genomics and Population Health (PHG Foundation), Cambridge, United Kingdom

There are many different forms of rare inherited disease that cause sudden cardiac death, arrhythmias and other cardiovascular conditions. In addition, a substantial number of other genetic diseases that affect different parts of the body are also associated with cardiological abnormalities. Together, individuals with these diseases represent a significant component of both cardiac and genetics services. In the last ten years, rapid advances in our understanding of the pathological basis of inherited cardiac disease, clinical features and associated risks, has created increasing potential for the effective clinical management of patients and their families. In particular, highly specialised services combining both cardiology and genetics expertise are required. The PHG Foundation has led a group of key experts and stakeholders - including clinical geneticists, cardiologists, electrophysiologists and other health professionals, health service commissioners and managers, and representatives from the British Heart Foundation, the Cardiomyopathy Association, the Arrhythmia Alliance, Cardiac Risk in the Young (CRY), Sudden Arrhythmic Death Syndrome (SADS UK) and the Marfan Association - in a review of national service provision in cardiac genetics in the UK. Findings will be presented including a description of the important features of good cardiac genetic services and related elements in primary and secondary care, pathology and coroner services. Current and projected future demand for these services and the potential impact of emerging knowledge and capabilities in the next few years will also be considered.

\section{SCREENING FOR TAY SACHS DISEASE IN ASHEKENZ} JEWISH SUB-POPULATIONS [51]

C.Alberg', H. Burton', A. Stewart', S. Levene', and C. Patch ${ }^{2}$

Foundation for Genomics and Population Health (PHG Foundation), Cambridge, United Kingdom

Guy's Hospital, London, United Kingdom

Tay Sachs disease (TSD) is an inherited degenerative neurological disease caused by deficiency of the enzyme hexosaminidase A. The most 
common form of the disease is lethal in infancy or early childhood, and there is no cure or effective treatment. The carrier frequency for TSD in Ashkenazi Jews is between 1 in 25 and 1 in 30, much higher than in the general population (about 1 in 250-300). In the United Kingdom, a screening programme for TSD has been in place since 1999. We report on work carried out by the PHG Foundation in conjunction with Guy's Hospital in London (the main centre for TSD screening in the United Kingdom) for the National Screening Committee: a needs assessment and review of current service provision for TSD screening in the UK. This project, which was informed by stakeholder consultation with patients and representatives from Jewish religious and community organizations on available services, not only highlighted strengths and weaknesses of the current system and how needs may evolve in the near future, but also examined alternative models for screening programs and included a review of TSD screening programs internationally, including in Australia, the US, Canada and Israel.

\section{AN UNEXPECTED PRESENTATION OF INTERSTITIAL} DELETION OF 140 IN A THREE GENERATION FAMILY [100]

M.Alcausin', F. Collins', C. Rudduck ${ }^{2}$, A. P. Darmanian'2, H. Martin ${ }^{3}$, and D. Sillence ${ }^{1,4}$ Departments of ${ }^{\prime}$ Clinical Genetics, ${ }^{2}$ Cytogenetics, ${ }^{3}$ Surgery, The Children's Hospital at Westmead, Westmead, New South Wales; ${ }^{4}$ Department of Paediatrics and Child Health, University of Sydney, Australia

We describe a three-generation family with five members having chromosome deletion (14) (q13q21). The male proband presented with history of antenatal ultrasound detection of ventriculomegaly and bilateral talipes equinovarus. Progress scans showed stable ventricular size. He was born full term with birth weight at 10th percentile and head circumference at 50th percentile. Examination at birth showed a generally non-dysmorphic child with bilateral hip dislocation and bilateral talipes. His anus was normally placed. Karyotype demonstrated 46, XY, del(14) (q13q21). At 3 months, persistent mild to moderate dilatation of the ventricles was noted on head ultrasound but head circumference was tracking appropriately. Review at 6 months of age revealed that he was developmentally normal. The proband's mother and two maternal aunts were known to the Clinical Genetics service as they all presented with oligodontia, fifth digit clinodactyly and variable severity of anterior placement of anus. Oligodontia was also present in the maternal grandfather but no anal anomaly. Chromosomal analysis done on the mother, aunts and grandfather showed the same chromosomal deletion. Psychomotor development and cognitive function in the members of the family with the deletion have been normal. Array analysis by comparative genomic hybridization is being undertaken to define the break points more precisely.

\section{AETIOLOGICAL CLASSIFICATION AND ANALYSIS OF BIRTH DEFECTS IN BABIES CONCEIVE USING ASSISTED REPRODUCTION (ART) [128]}

\section{D.Amor ',2, A. Jaques', D. Healy ${ }^{3}$, S. Breheny ${ }^{4}$, G. Baker ${ }^{4}$, and J. Halliday 1,2}

Murdoch Childrens Research Institute, Parkville, Australic

Department of Paediatrics, University of Melbourne, Australia

Department of Obstetrics and Gynaecology, Monash University, Clayton, Australia

Department of Obstetrics and Gynaecology, University of Melbourne, Australia

Babies conceived using assisted reproduction technologies (ART) have a $30 \%$ increased likelihood of being born with a birth defect, however causative pathways are unknown. Previous studies have grouped birth defects in ART using anatomical classifications (ICD-9), potentially masking etiological information. We hypothesized that greater insight into the etiology of birth defects in ART could be gained by using a classification based on etiology. We reclassified all birth defects in a cohort of 7,613 ART conceptions and 25,389 non-ART controls from a retrospective record linkage cohort study covering the years 1991-2004. All birth defects were reclassified using a hierarchical system comprising eight etiological categories. Birth defects of unknown etiology were further classified according to whether the embryological origins of the birth defect(s) could be traced to blastogenesis. Compared to non-ART controls, ART babies were more likely to have a birth defect of unknown etiology (OR 1.39, 95\% CI 1.23-1.57), but not more likely to have a birth defect of known genetic etiology (OR 1.10 , 95\% CI 0.81-1.48). ART babies were more likely to have multiple birth defects (OR $1.88,95 \% \mathrm{CI}$ 1.19-2.95) and defects of blastogenesis (OR 2.04, 95\% CI 1.45-2.87) compared to non-ART controls. Our data suggest that the increase in birth defects in ART pregnancies is the result of an increase in multiple malformations, blastogenesis defects and birth defects without known genetic etiology rather than known genetic defects, and support the hypothesis that the increase in birth defects in ART babies is due to environmental or epigenetic factors resulting from the ART process.

\section{CONSIDERING POPULATION CARRIER SCREENING: THE IMPORTANCE OF LIFE EXPERIENCES [64]}

\section{A. D. Archibald ${ }^{1,2}$, B. J. McClaren ${ }^{1,2}$, and S.A. Metcalfe ${ }^{1,2}$}

${ }^{\prime}$ Murdoch Childrens Research Institute, Australia

2 Department of Paediatrics, The University of Melbourne, Australia

Population carrier screening is currently being offered and investigated for a number of genetic conditions. There is a need to understand factors influencing individuals' consideration of screening tests. We discuss a common theme identified in two independent studies using a qualitative approach to explore attitudes to population carrier screening for fragile $\mathrm{X}$ syndrome (FXS) and cystic fibrosis (CF). Focus groups and interviews were conducted with a range of stakeholders including: pregnant and nonpregnant women, relatives of individuals with the condition, healthcare providers and community members. There were 205 participants in the FXS study and 68 participants in the CF study. In both studies, life experience was identified as an important factor influencing an individual's interest in screening. Participants discussed: the lack of awareness of FXS and $\mathrm{CF}$ in the general population; that a lack of experience with healthrelated issues may result in a perception that screening is irrelevant and that when considering screening, individuals may draw on their own life experiences to inform their decision-making. These findings indicate there are members of the general population who may be unlikely to consider screening and for those who do consider screening, making a decision may be challenging because of a lack of experience with the genetic condition. Those who are considering screening will need to be provided with detailed information about the condition from a variety of sources. When offering screening, healthcare providers need to view individuals in the context of their life experiences in order to understand and facilitate their decision-making.

\section{POPULATION CARRIER SCREENING FOR FRAGILE $X$ SYNDROME: CHALLENGES FOR GENETIC COUNSELLING [65]}

A. D.Archibald ${ }^{1,2}$, S.Wake $^{3}$, A. M. Jaques', C. Hickerton', and S.A. Metcalfe ${ }^{1,2}$

${ }^{1}$ Murdoch Childrens Research Institute, Australia

${ }^{2}$ Department of Paediatrics, The University of Melbourne, Australia

${ }^{3}$ Genetic Health Services Victoria, Australia

${ }^{4}$ Fragile X Alliance Inc, Australia

Fragile X syndrome (FXS) is the leading cause of inherited intellectual disability. Currently, population carrier screening for FXS is being investigated as it can provide carriers with information about their health and their risk of having a child with FXS. This study explored attitudes to population carrier screening for FXS. Here we discuss the relevance of these findings to genetic counselling. Interviews and focus groups were conducted with 172 participants: healthcare providers (112), relatives of individuals with FXS (29) and women from the general population who had been offered carrier screening for FXS (31). Participants discussed a range of issues relevant to genetic counselling including: challenges an individual may face when making a decision about carrier testing for a condition they have not experienced; their perceptions that the test is most relevant to those planning families; their views on the role of genetic counselling in a population carrier screening program for FXS. The results highlight the importance of ensuring individuals have the opportunity to reflect on their experiences with disability to help inform their decisionmaking, in addition to providing detailed information about FXS and its impact on affected individuals and their families. Furthermore, individuals should be supported to consider when might be the best time in their lives to learn their carrier status. These results provide valuable insight into the challenges facing genetic counsellors offering population carrier screening for FXS and will help inform future development of genetic counselling practice in this context.

\section{COPY NUMBER VARIATIONS IN THE FOUNDER GYPSY POPULATION [160]}

D. N.Azmanov', B. Morar', D. Angelicheva', I.Tournev' ${ }^{2,3}$, R. Kaneva ${ }^{4}$, J.W Sander ${ }^{5}$ A. Jablensky ${ }^{6}$, M. Bahlo ${ }^{7}$, and L. Kalaydjieva

Laboratory for Molecular Genetics, Centre for Medical Research and Western Australian Institute for Medical Research, The University of Western Australia, Perth, Australia Department of Neurology, Medical University, Sofia, Bulgaria

Department of Cognitive Neuroscience, New Bulgarian University, Sofia, Bulgaria ${ }^{4}$ Molecular Medicine Centre, Medical University, Sofia, Bulgaria Department of Clinical and Experimental Epilepsy, University College London, United Kingdom

${ }^{6}$ School of Psychiatry and Clinical Neurosciences, The University of Western Australia, Perth, Australia

Bioinformatics Division, The Walter and Eliza Hall Institute of Medical Research, Melbourne, Australia

Recent advance in whole genome genotyping technologies with the detection of copy number variations (CNVs) have introduced a new dimension to our understanding of human variation in health and disease. Studies 
into populations other than the intensively studied HapMap samples will show the full spectrum of CNVs. Taking into account population specificity, CNV identification will help the discrimination between benign and disease-associated variants. We have studied the founder Gypsy population for more than a decade, identifying the genetic basis of many Mendelian diseases, and recently focusing onto complex disorders in this population. For CNV analysis, using Affymetrix SNP Array 5.0 and PennCNV algorithm, we have studied 30 unrelated controls of Gypsy ethnicity. We have observed $251 \mathrm{CNV}$ s with ca.75\% of those coinciding with CNVs registered in the Database of Genome Variants (DGV) (http:// projects.tcag.ca/variation/) and more than $84 \%$ being rare (frequency < 0.05 ). The overlapping loci included genes for immunoglobulins (IGH, IGK, IGL), T-cell receptor genes, killer-cell immunoglobulin-like receptors, MHC class I and II, centromeric and telomeric regions. Contrary to HapMap data, population-specific CNVs in the Gypsies did not affect genes responsible for olfaction (olfactory receptors), drug metabolism (CYP2D6), HIV resistance (CCL3L1), and immunoglobulin-binding $(F C G R 3 B)$. Among the population-specific CNVs, 32 were located in gene deserts, 13 were intronic, and 17 overlapped UCSC genes. Characterised genes from the latter group are involved in inflammatory response, DNAbinding, protein transport, and tRNA processing. Future disease studies in the Gypsy population will rely on information from both DGV and the present study.

\section{ONE FAMILY, TWO TRANSLOCATIONS AND THREE DELETIONS [134]}

S. Bain', A. Baxendale', T. Hocking', J. Nicholl', W.Waters', and E.Thompson Cytogenetics Unit ${ }^{l}$ and South Australian Clinical Genetics Service ${ }^{2}$, SA Pathology, Women's and Children's Hospital, North Adelaide, Australia

A female infant born at 33 weeks gestation with polyhydramnios, small kidneys and Pierre Robin sequence was referred for chromosome analysis. GTL banding at 550 band resolution showed that this baby had not one but two reciprocal translocations; $\mathrm{t}(5 ; 12)(\mathrm{p} 13.3 ; \mathrm{p} 12.2)$ and $\mathrm{t}(6 ; 14)$ (?q24.2;p11.2). Higher resolution banding at 800 bands showed that the $\mathrm{t}(5 ; 12)$ appeared apparently balanced, however a question was placed on the der(6) involved in the $t(6 ; 14)$ as there was difficulty in accurately resolving breakpoints. Parental chromosome studies were requested which prompted laboratory discussion of the possibility that each parent may carry a translocation or a de novo event had occurred in our patient. Surprisingly, the mother of this child was found to carry both translocations, which have then been inherited by her daughter. Physical examination of the mother showed that she has an unusual facial appearance, with micrognathia, a broad forehead, a broad nasal tip and exopthalmus of the left eye, which may have been related to her Graves disease. Her intelligence has not been formally assessed but appears to be low average or borderline. Chromosome studies of grandparents are not available. Microarray studies using an 'in house' custom whole genome microarray detected three non contiguous deletions in the long arm of chromosome 6 at 6q14.1-q14.4, 6q14.3-q15 and 6q15. No other deletions were detected at other translocation breakpoints. FISH studies were performed on both mother and daughter and both were deleted for the same FISH probes.

\section{PERINATAL HYPOPHOSPHATASIA PRESENTING AS NEONATAL ONSET SEIZURES [83]}

\section{S. Balasubramaniam \\ Western Sydney Genetics Program, The Children's Hospital at Westmead. New South Wales, Australia}

Hypophosphatasia is a rare inherited metabolic disorder characterized by defective bone and teeth mineralization, and deficiency of alkaline phosphatase activity in serum and tissues. The disease is caused by mutations in the liver/bone/kidney alkaline phosphatase gene (ALPL; OMIM\#171760), encoding tissue non specific alkaline phosphatase (TNSALP) and has extensive allelic heterogeneity leading to a range of clinical phenotypes. The enzyme cleaves extracellular substrates; pyridoxal-5-phosphate, phosphoethanolamine and inorganic pyrophosphates. Based on age and clinical presentation, six clinical forms are currently described; perinatal lethal, perinatal benign, infantile, childhood, adult and odontohypophosphatasia. The birth prevalence of severe forms is 1:100000. We report an infant born to non consanguineous parents diagnosed with lethal hypophosphatasia and a unique presentation of neonatal onset seizures, severe encephalopathy, autonomic instability and central hypoventilation. The diagnosis of hypophosphatasia was based on radiological findings, low serum alkaline phosphatase and grossly elevated urinary phosphoethanolamine. Mutation analysis of the ALPL gene is presently being sought for genotype-phenotype correlation as her severe extraskeletal complications did not concur with the milder radiographic presentation, consistent with the benign perinatal form. Biochemical analysis of her cerebrospinal fluid showed an elevated phosphoethanolamine, a possible epileptogenic, natural substrate of TNSALP and reduced 5HIAA, biopterin levels, compatible with secondary intracellular pyridoxine deficiency. These findings correlate with the critical indirect function of TNSALP on the regulation of intracerebral pool of pyridoxal -5 phosphate and its dependant enzymes, crucial in neurotransmitter metabolism Mutation analysis will confirm her diagnosis and determine if molecular heterogeneity contributes to the clinical variability of her presentation.

\section{MOVING WITH THE TIMES AND THE EVIDENCE IN THE TRANSLATION OF GENETICS KNOWLEDGE: 20 YEARS OF GENETICS EDUCATION [103]}

K. Barlow-Stewart ${ }^{1,2}$, M. Saleh', and K. Dunlop'

Centre for Genetics Education, NSW Health, Royal North Shore Hospital, Sydney, Australia 2 Faculty of Medicine, University of Sydney, Sydney, Australia

The Centre for Genetics Education as a statewide service of NSW Health has been providing information for professionals, individuals, families and the wider community as core business since its foundation in 1989. A wide range of resources have been built up, with content developed and updated as a result of partnerships between experts and the community. Quantitative and qualitative methodologies have guided the development and targeting of resources, modes of delivery of information and the evolution of other educational strategies from traditional print-based formats and didactic presentations to incorporating multimedia, web-based and interactive strategies. Such changes have required adaptation in staff skills from primarily genetic counseling to those specializing in health promotion and communicating health across media such as the Internet. Concomitantly the role of the Centre has evolved as an advocate with a focus on the societal impact of the technologies such as discrimination and proactive use of the print media, radio and television in communicating genetic health issues to the wider community. The paper describes the challenges of an education program balancing the maintenance of information and support for those affected by often rare genetic conditions as a referral and information partner in the NSW Genetics Service with informing the community and professionals in all fields of the implications of the increasingly wider, and often nonmedical applications of genetics technologies so that informed decisions regarding its use can be made. Lessons learnt over time in meeting these challenges may assist other providers of genetics education.

\section{GENETICS AND PUBLIC HEALTH OPPORTUNITIES FOR HEALTH PROMOTION AND PREVENTION: EDUCATING THE NON-GENETICS PROFESSIONAL [105]}

\author{
K. Barlow-Stewart ${ }^{1,2}$ and B.Armstrong ${ }^{3}$ \\ 'Centre for Genetics Education, Royal North Shore Hospital, Sydney, Australia \\ ${ }^{2}$ Faculty of Medicine, University of Sydney, Australia \\ School of Public Health, University of Sydney, Australia
}

The balance that must be struck between the uses of genetic information made by the individual, family and wider societal collectives requires knowledge of genetics science and its applications and ethical, regulatory, legal and social implications by a range of professionals involved in policy and decision-making. These include practitioners, students and researchers in public health, policy, journalism, law, epidemiology, medicine, science, industry, ethics, actuarial and insurance disciplines, philosophy, communication and advocacy. To meet this need a 3-day workshop 'Genetics and Public Health' has been conducted with this group by the School of Public Health, University of Sydney since 2001, following Australian Government support for its development under the Public Health Education and Research Program. Some 300 have attended: most using the unit as an elective for their Master of Public Health. Three case studies covering cystic fibrosis, breast and ovarian cancer and haemochromatosis underpin both didactic and interactive learning modes to provide students with knowledge of the basic study designs to determine how strongly a disease is genetically determined, the contribution of gene and environment interaction to its cause and an appreciation of the potential that genetics and genetic research has to prevent disease and promote health. Results of evaluations conducted 2001-2008 will be presented which show that the participants rate the course highly for content and format as well as relevance to their current or intended workplace or discipline.

\section{DEVELOPMENT OF COUNSELLING BEST PRACTICE STANDARDS FOR TERMINATION OF PREGNANCY FOR FETAL ABNORMALITY [217]}

\section{A. Baxendale}

South Australian Clinical Genetics Service, SA Pathology, Women's and Children's Hospital, South Australia, Australia

One of the initiatives of the South Australian Government's Women's Health Action Plan in 2006/07 was to develop best practice standards for termination of pregnancy. The standards were to cover clinical practice, counselling and support services and to include a plan for implementation of these standards in SA. Termination of pregnancy for fetal abnormality accounts for approximately $2-3 \%$ of the total number of terminations in 
SA annually. In the past the services offered to women varied considerably on the basis of where they were receiving their health care, the type of abnormality diagnosed and the woman's response to the diagnosis and her request for information and counselling. The development of best practice standards was seen as an opportunity to develop specific guidelines on counselling and support to ensure that all women in SA are offered a similar service. Developing the guidelines involved representatives from relevant health institutions meeting regularly. With increased awareness of the services offered by the different health institutions, significant changes in service delivery occurred in the short term prior to the implementation of the best practice standards. These changes related to an increased number of women being offered nonmedical counselling and a choice of method of termination of pregnancy where appropriate. The best-practice standards regarding counselling, provision of written information and follow-up and support are discussed along with the role of genetic counsellors in ensuring that these standards are met.

\section{EVALUATION OF THE PRENATAL SCREENING AND} DIAGNOSTIC TESTS PATIENT INFORMATION BOOKLET [193]

S. Baxendale, S.Appleton, and P. O'Leary

Office of Population Health Genomics, Department of Health Western Australia, Australia

A key component in the translation of genomics knowledge into health benefits is the development of educational resources and programs. One of the resources developed by the OPHG in 2005 is a Prenatal Screening and Diagnostic Tests booklet. This resource combined five screening and diagnosis booklets into a single booklet to assist pregnant women in their decision-making about first trimester screening. To ensure the Prenatal Screening and Diagnostic Tests booklet was still relevant and current, an evaluation was undertaken during June to December 2008. The evaluation comprised three components: a review of the literature to identify best practice in the development of patient health information, a survey of health professionals, and a survey of patients attending a metropolitan private ultrasound practice for a fetal anatomy survey (at approximately 19 weeks gestation). In total, 19 health professionals and 30 patients completed evaluation surveys. Separate surveys for the health professionals and patients were developed which incorporated validated scales. The evaluation assessed the booklet on content, presentation and readability. Results from this evaluation will guide the development of best practice guidelines for resource development and improve the Prenatal Screening and Diagnostic Tests booklet for health professionals and patients.

INTRODUCTION OF ARRAY COMPARATIVE GENOMIC HYBRIDISATION (ACGH) INTO A DIAGNOSTIC LABORATORY (GENETIC SERVICES, WELLINGTON HOSPITAL) [77]

R. Beddow, M. Smith, J. Dixon, and C. Felix

Central and Southern Genetic Service, Wellington Hospital, New Zealand

Since it was first described in 1987 microarray technology has been developed for a wide variety of research and diagnostic uses. The completion of the Human Genome project in 2003, facilitated rapid advances in this technology, the availability of commercial platforms and high quality software has led to the possibility of utilising microarray platforms in routine genetic testing. We have assessed some of the platforms available and conducted in-house and commercial validation studies. DNA samples were processed using the Agilent, Affymetrix and Niblegene platforms and subsequently fifty samples have been processed using the Agilent $4 \mathrm{x} 44 \mathrm{~K}$ aCGH platform and G2565AA/BA Microarray Scanner system within our laboratory. These studies have shown an approximately $20 \%$ abnormality rate including a variety of previously undiagnosed duplications/deletions and examples of the abnormalities detected will be considered. Various array, molecular and cytogenetic options are now available for screening dysmorphic, developmentally delayed children. The rational for an integrated testing strategy within our service will be discussed.

\section{CONSANGUINITY AS A RISK FACTOR IN THE GLOBAL BURDEN OF DISEASE [57]}

A. H. Bittles on behalf of the Consanguinity Risk Factor Group.

Centre for Comparative Genomics, Murdoch University, Western Australia, Australia

The Global Burden of Disease Study (GBD) is currently being conducted with completion scheduled for 2010. The GBD will comprise data on $>180$ disease states and injuries collected on country, regional and global bases. The ultimate aim is to produce comparative information on the burdens imposed by different diseases, in part presented as Disability Adjusted Life Years (DALYS). Two groups of genetic disorders are included in the GBD, congenital disorders and haemoglobinopathies. In addition, consanguinity has been incorporated as a specific risk factor and will be included in the overall data analysis. To assess the possible role(s) of consanguineous marriage, information on a wide range of disease states has been collated from published and unpublished sources $(n>10,000)$. Prior to formal inclusion in the preliminary analysis, all reports were subjected to a series of rigorous acceptance/rejection criteria. The analysis has been subdivided into four categories: reproductive behavior and fertility; morbidity — for example, congenital heart defects, oral and facial clefts, haemoglobinopathies, and adult-onset disorders; mortality, - for example, stillbirths, and deaths in infancy, childhood and adulthood; social and economic correlates of intrafamilial marriage, including female autonomy and divorce. Consanguineous marriage remains popular across many regions of the world and is estimated to involve more than $15 \%$ of the current global population. Given the significant impact of consanguinity on health reported in many previous investigations, the unparalleled scale and range of the present study is providing invaluable information on this important topic.

\section{NONINVASIVE PRENATAL DIAGNOSIS OF TRISOMY 21- INCREASING THE INFORMATIVENESS OF THE PLAC4} MARKER [74]

T. Bose, C. Nicholls, J.Dodd, P. Muller, J. Robinson, and S. Yu

Department of Molecular Genetics and Cytogenetics (WCH), SA Pathology, Australia Department of Obstetrics and Gynaecology, Women's and Children's Hospital, Adelaide, South Australia, Australia

Routine prenatal diagnosis requires amniocentesis or chorionic villus sampling (CVS), which are invasive and carry a 1-2\% risk of miscarriage. Noninvasive prenatal diagnosis (NIPD) has been explored. We previously reported the identification of a novel polymorphic marker in the PLAC4 gene. Analysing allelic ratio of this marker using fetal PLAC4 RNA in maternal plasma differentiated trisomy 21 from non-trisomy pregnancies. Further analysis of this marker using capillary electrophoresis (CE) identified a third allele. By analysing 50 bloodbank DNA samples, the frequencies of the three alleles were established to be $0.73,0.23$ and 0.04 respectively. Based on these, it is estimated that $41 \%$ of all pregnancies and $60 \%$ of the trisomy pregnancies will be informative using this marker alone. Investigation is on going with the potential to identify more alleles and to further increase the informativeness of this marker. In addition, DNA sequences of the minor allele $(4 \%)$ match that initially published in NCBI. This could have happened purely by chance. Alternatively, the allele frequencies could be different in other populations. If the minor allele is more than $4 \%$ in other populations, this marker will be more informative and therefore more useful in those populations. We will investigate the allele frequencies in other populations.

\section{A REVIEW OF LYMPHOMA TESTING BY CONVENTIONAL CYTOGENETICS AND FLUORESCENCE IN SITU HYBRIDISATION (FISH) [131]}

T. Bowen', T. Grumball', F. Price', J. Peverall', J. Loane', B. Amanuel'², D. Spagnolo², and A. Murch'

' Cytogenetics Department, PathWest Laboratory Medicine WA, King Edward Memorial Hospital, Subiaco, Western Australia, Australia

Anatomical Pathology, Division of Tissue Pathology, PathWest Laboratory Medicine WA, QEII Medical Centre, Nedlands, Western Australia, Australia

A review was made of all lymphoma samples received from a single source (Anatomical Pathology, Division of Tissue Pathology, PathWest Laboratory Medicine WA, QEII Medical Centre). by the PathWest Cytogenetics Department at King Edward Memorial Hospital (WA) during the past two years. Fresh specimens for culture were used, wherever possible, for banded metaphase analysis. Imprint slides were made on all fresh specimens to be used for targeted FISH on request. When no fresh specimen was available formalin fixed paraffin embedded slides were sent in cases where FISH was considered likely to help diagnosis or prognosis. The purpose of this review was to determine the most efficient and effective method of identifying diagnostically relevant results. FISH and metaphase analysis were compared on the basis of success rate in detecting abnormalities, time taken to get a result, cost. Culture failure, and cost were major problems for metaphase analysis, whereas while FISH rarely failed to work, targeted FISH did not always detect relevant abnormalities.

\section{MEASURING THE IMPACT OF GENETIC DISEASE IN THE WA POPULATION [175]}

K. Brameld', S. Maxwell', D. Dye', P. O'Leary', J. Goldblatt', C. Bower ${ }^{3,4}$,

H. Leonard ${ }^{4}$, J. Bourke ${ }^{4}$, and E. Glasson ${ }^{5}$

Office of Population Health Genomics, Department of Health, Australia

Genetic Services of Western Australia, Australia

Birth Defects Registry, King Edward Memorial Hospital, Australia

Telethon Institute for Child Health, Western Australia, Australia

School of Population Health, The University of Western Australia

The WA Data Linkage System was used to measure the burden of genetic disease resulting from single gene or chromosome disorders in Western Australia as defined by cases receiving inpatient hospital care. ICD codes for single gene and chromosomal disorders were identified in consultation with relevant experts. All hospital inpatient records with these codes in any diagnosis field were extracted from the WA Data Linkage System 
together with any other linked hospital records for the period 1980-2006. The resulting dataset was analysed to determine trends in the number of people admitted, number of admissions, length of stay and cost of admissions for single gene and chromosome disorders in the WA population. Results were validated by use of the Birth Defects Registry and the IDEA (intellectual disability exploring answers) database. The study will provide information on the burden of genetic disease in terms of single gene and chromosome disorders in Western Australia and the associated use of hospital services. This information will be used to inform service and policy development including the requirement for genetic testing, screening and counseling services according to demographic factors. The study will also provide data for the evaluation of genetic health services.

\section{AN EVALUATION OF PRENATAL SCREENING STRATEGIES IN THE AUSTRALIAN CONTEXT [182]}

\section{K. Brameld', P. O'Leary', and R. Cocciolone \\ Office of Population Health Genomics, Department of Health, Western Australia, Australia 2 Department of Genetic Medicine and Department of Genetic Medicine, University \\ of Adelaide, South Australia, Australia}

Recent years have seen the introduction of two-stage prenatal screening strategies which have improved performance characteristics than those of first or second trimester screening alone, but come at an increased running cost. This paper compares the costs and looks at the pros and cons of introducing two-stage screening methods. A decision analysis model was constructed to evaluate outcomes of the following prenatal screening options: (1) first trimester combined screen, (2) second trimester triple screen, (3) second trimester quadruple screen, (4) contingent screen, (5) sequential screen and (6) integrated screen, in the Western Australian context. The results indicate that contingent screening was the most effective screening method in terms of cost and performance characteristics. However, the goal of offering two-stage prenatal screening in Australia must be compared with that of improving access and education for the current service.

\section{TOWARDS IMPLEMENTATION OF NON-INVASIVE PRENATAL} DIAGNOSIS IN THE UK NATIONAL HEALTH SERVICE [33]

\section{P. Brice, H. Burton, and C.Wright \\ Foundation for Genomics and Population Health (PHG Foundation), Cambridge, United Kingdom}

Emerging technologies that capitalize on the presence of cell-free fetal nucleic acids in the maternal bloodstream have the potential to transform prenatal diagnosis and care by allowing non-invasive testing from as early as 7 weeks. This represents a major advance, since diagnosis currently requires a later, invasive procedure (amniocentesis or chorionic villus sampling) with an associated miscarriage risk of around 1\%. Many women opt against prenatal testing for this reason, and hundreds of healthy fetuses are lost following testing each year in the United Kingdom alone. Technological applications already in active development are the determination of fetal sex or the diagnosis of specific diseases in the fetus of women at high risk of inherited disease; the determination of fetal Rhesus blood-group status in Rhesus-negative women at high risk of complications of pregnancy with a Rhesus-positive fetus; and the diagnosis of aneuploidies such as Down Syndrome in the fetus. The approach may permit diagnosis of a wider range of genetic diseases in the future, subject to certain limitations, and ultimately could also allow improved monitoring for complications of pregnancy such as pre-eclampsia. The PHG Foundation has led an expert working group of key UK stakeholders, including National Health Service (NHS) researchers, clinicians, managers and patient representatives, in an examination of current evidence and associated ethical, legal and social issues in this area. Findings and strategic recommendations for the effective and responsible implementation of this new technique into health services in the UK will be presented.

\section{A CASE OF MOSACISIM FOR TRISOMY OF CHROMOSOME 5 DETECTED AT AMNIOCENTESIS [136]}

S. Brown', S. Higgins', R. Hutchinson', S. Bain', L. Moore², and E. Haan ${ }^{3}$

Cytogenetics Unit ${ }^{1}$, Histopathology Department ${ }^{2}$ and South Australian Clinical Genetics Service ${ }^{3}$, SA Pathology, Women's and Children's Hospital, North Adelaide, Australia

A 33-year-old G1P0 woman was referred from the country at $21+3$ weeks gestation for a second opinion ultrasound scan. First trimester antenatal screening results based on biochemical and nuchal translucency measurements indicated that the fetus was not at increased risk of Down or Trisomy 18 syndromes (1:4539 and 1:3550 risks respectively). Ultrasound examination suggested a cardiac abnormality, agenesis of the corpus collosum and a cloverleaf abnormality of the skull. Examination at autopsy confirmed these abnormalities. Karyotyping of amniotic fluid showed a mosaic female karyotype with trisomy for chromosome 5 observed in four independent cultures and in $50 \%$ of the colonies examined. After termination of pregnancy, placental and fetal tissues were cultured, karyotyped and examined for the presence of trisomy 5 cells. These were found in all tissues except chorionic villi and fetal blood. Mosaicism for trisomy 5 has rarely been reported at prenatal diagnosis. In most cases reported the trisomic line is confined to the placenta and is not found in fetal tissues. In this case the trisomic cells were not found in chorionic villi and as a result were unlikely to be detected via an earlier CVS procedure. The combination of ultrasound technology and insitu culturing of amniotic fluid resulted in the diagnosis of true mosaicism in this fetus.

\section{STRATEGIC USE OF DELIBERATIVE PUBLIC ENGAGEMENT} IN BIOBANK GOVERNANCE [300]

M. M. Burgess, K. O'Doherty, and D.Winickoff

W. Maurice Young Centre for Applied Ethics and Department of Medical Genetics Principal, College for Interdisciplinary Studies, University of British Columbia, Vancouver Canada

The policy literature increasingly supports forms of direct public or participant input into the governance of biobanks and our research collaborations have demonstrated that it is possible to derive policy advice from deliberative engagement. Three rationales address different aspects of the governance of biobanks, and suggest different strategic roles for deliberative engagement. (1) Biobanks will influence many features of society in the future and require public investment associated with lost opportunity costs. This may justify enhanced participation of publics unfiltered through institutions and groups. (2) The governance of biobanks requires trade-off choices between social goods that are highly valued for their own sake. This may justify facilitated, deliberative engagement of stakeholders and those speaking in behalf of the various social goods like privacy, collective health benefit, enhanced competitiveness, and protection from racism and discrimination. (3) The constitution or membership of a biobank requires individual donors to contribute samples, data and often to be active in prospective development of a powerful research resource. The aggregation of samples and data may establish a collective entitlement to participate in the control of the biobank that may justify representation of the individuals associated by participation in the biobanks. Informed, deliberative engagement might make this participation more meaningful and representative. Recruitment for each of these purposes needs to be carefully distinguished and has implications for the design of the informed deliberation.

\section{GENETIC COUNSELLING RECOMMENDED: IDENTIFICATION} OF A DELETION OF THE EYA1 GENE BY CGH MICROARRAY IN A CHILD WITH BRANCHIO-OTO-RENAL SYNDROME [16]

\section{J. Burgess}

Nepean Hospital, Sydney West Area Health Service, Australia

$\mathrm{CH}$, an 18-month-old boy was originally referred to the genetics service by an ENT surgeon in 1998. He had clinical features and a paternal family history suggestive of branchio-oto- $\mathrm{CH}$, an 18-month-old boy was originally referred to the genetics service by an ENT surgeon in 1998. He had clinical features and a paternal family history suggestive of branchio-otorenal syndrome. Branchio-oto-renal (BOR) syndrome is characterised by malformations of the inner, middle and outer ear associated with sensorineural, conductive or mixed hearing impairment; branchial fistulae and cysts; and renal malformations, ranging from mild renal hypoplasia to bilateral renal agenesis. BOR is an autosomal dominant condition with variable expression, but high penetrance. Hearing loss has been reported in approximately $75 \%$ of cases and renal anomalies occur in about $10 \%$ of affected individuals. The diagnosis of BOR is made using clinical criteria. It is a heterogeneous condition. Molecular genetic testing of the EYAl gene located on chromosome 8 detects mutations in approximately $40 \%$ of individuals with the clinical diagnosis of BOR syndrome. Molecular genetic testing of the SIX5 gene located on chromosome 19 detects mutations in $5 \%$ of individuals with the clinical diagnosis of BOR syndrome who do not have an EYA1 mutation. $\mathrm{CH}$ and his affected father had blood taken for research into BOR in 1998 with no research results to date. In 2008 a CGH microarray was conducted and a microdeletion was detected in $\mathrm{CH}$ at 8q13. This deletion includes the entire EYA1 gene. This deletion was also found in his affected father. The psychological impact of discovering the mutation ten years later will be explored as well as its implications on cascade testing in the family.

\section{GENETIC TEST EVALUATION: EXPLORING CLINICAL UTILITY IN OPTHALMOLOGY [52]}

\section{H. Burton', T. Moore ${ }^{2}$, S. Downes ${ }^{3}$, and S. Carless ${ }^{4}$}

Foundation for Genomics and Population Health (PHG Foundation), Cambridge, United Kingdom

UCL Institute of Opthalmology, London, United Kingdom

Oxford Eye Hospital, Oxford, United Kingdom

${ }^{4}$ Birmingham Hospital, Edgbaston, United Kingdom

The ACCE evaluation framework for genetic test evaluation provides a model process for evaluation with clinical utility as one of its core features. 
This framework was expanded by Burke and Zimmern in a report entitled 'Moving Beyond ACCE' to identify components of clinical utility that build on the different purposes of genetic testing, the way in which genotype contributes to disease, and other dimensions of health care quality. As part of a Working Group on Genetics in Opthalmology set up by the UK Genetic Testing Network, the concepts were explored by consideration of particular conditions and the use of case histories. This supported the view that the purposes of genetic testing were to provide: benefits of information from better diagnosis and prognosis; decreasing morbidity and mortality by preventative care and informing treatment decisions; improving the process of care; and information to assist reproductive choice. The utility of a molecular diagnosis in addition to a clinical diagnosis must be justified. However, with the rarity of these conditions and the diversity of outcomes sought be patients and families depending on their circumstances, it was concluded that formal evaluations could not be required for all tests. The proposal by the UKGTN that test criteria should be established for access to tests is supported.

\section{DETECTION OF RARE EVI1 REARRANGEMENTS IN THREE} PATIENTS WITH ACUTE MYELOID LEUKAEMIA [159]

\section{N. Buzzacott', J. O'Reilly', N. Egan',V. Britton', R. Herrmann',A. McQuillan²,} and S.Ward ${ }^{3}$

Royal Perth Hospital, Perth, Western Australia, Australia

Fremantle Hospital, Perth, Western Australia, Australia

Sir Charles Gairdner Hospital, Perth, Western Australia, Australia

The inv(3)(q21q26) and $\mathrm{t}(3 ; 3)(\mathrm{q} 21 ; \mathrm{q} 26)$ are two cytogenetic rearrangements that result in the inappropriate expression of the gene EVI1. The Ecotropic Viral Integration Site 1 (EVI1) gene encodes a zinc finger protein usually involved in cell migration, growth and differentiation. This gene is not normally expressed in haematopoietic cells, however the inv(3) and $t(3 ; 3)$ bring about the juxtaposition of EVI1 with the enhancer of the constitutively expressed RPN1. This inappropriate expression alters cell differentiation, and promotes the proliferation of granulocytes, erythrocytes and megakaryocytes. Both rearrangements have been described in patients with myelodysplastic syndrome, acute myeloid leukaemia and the transformation phase of chronic myeloid leukaemia. These rare rearrangements are important to detect, as they are associated with a poor prognosis. We present three cases where Fluorescence in-situ Hybridisation analysis indicated the involvement of EVI1 in rearrangements other than the typical cytogenetic presentation. These rearrangements are a translocation between chromosomes 3 and 8 within a complex karyotype, an inv(3q) not involving the q21 breakpoint and a complex rearrangement of both chromosome 3s. These cases indicate that EVI1 may be implicated in a variety of rearrangements, and that FISH analysis can be helpful in determining the nature of these abnormalities.

\section{POPULATION GENETICS AS A TOOL FOR PUBLIC HEALTH: EXAMPLE OF HEMATOPOIETIC STEM CELL PROCUREMENT FOR TRANSPLANTATION [169]}

A. Cambon-Thomsen, P.A. Gourraud, and the POSEIDON project

Inserm, U 558 \& University of Toulouse, Epidemiology and Analyses in Public Health, Faculty of Medicine, Toulouse, France

The knowledge of HLA diversity distribution in populations has become today essential for planning the constitution and use of donor Registries or cord blood banks for allogeneic haematopoietic stem cell transplantation (HSCT); especially the use of estimated haplotype frequencies is becoming an essential parameter for planning decisions in this health sector, as well as for individual decisions regarding the choice of the best compatible donor for a given patient. The importance of HLA compatibility for HSCT has been known for long and its complexity has been increasing over years. Today, more than 12 million potential donors of HSC or Cord blood bank units are available worldwide for patients without a family donor. Whereas haplotype frequencies are classical parameters of population genetics, their direct application in the HSCT domain has become a reality in three aspects at least: (1) estimation of the chances for a patient to find a suitable donor given its HLA type; this may influence the therapeutic options; (2) estimation of registries suitable size and strategy for recruiting donors or for establishing cord blood banks in given populations in order to better match the patients needs; (3) estimation of compatibility at haplotype level that leads to a better clinical outcome as compared to non phased information. In view of such developments refining the haplotype estimation methods and finding solutions to better overcome the global accuracy evaluation of haplotype frequencies has become of practical interest and such issues are not anymore only restricted to statistical geneticists. (See http://www.poseidon-hsct.eu/)

\section{THE STATUS OF GENETIC INFORMATION IN HEALTH} SYSTEMS: A EUROPEAN VIEW [75]

A. Cambon-Thomsen, S. Julia, A. Pigeon, and E. Rial-Sebbag

Inserm, U 558 \& University of Toulouse, Epidemiology and analyses in public health, Faculty of Medicine, 37 allées Jules Guesde, 31400 Toulouse, France

Genetic information can be considered as sensitive personal information or health information and is regulated as such in many health systems. In a number of cases its production and uses are specifically regulated by law or other kind of regulatory texts. There is a huge variability in the kind of regulations that apply to genetic information in European countries and we analyse the relevance and influence of supra-national texts in such regulations. The most recently adopted text on this topic is at the Council of Europe level, namely, the Additional Protocol to the Convention on Human Rights and Biomedicine, concerning Genetic Testing for Health Purposes' Strasbourg, 2008 (http://conventions.coe.int/Treaty/en/Treaties/ $\mathrm{Html} / 203$.htm); it specifies a number of points complementary to those addressed in the 'Convention on Human Rights and Biomedicine',1997 (http://conventions.coe.int/treaty/EN/Treaties/Html/164.htm). We will analyse this document in relation to the situation in some chosen European countries (including France, Spain, and UK), especially with regard to the definitions used and the principles applied, and with reference to the 25 recommendations published by the European Commission in 2004 (http://ec.europa.eu/research/conferences/2004/genetic/) following the work of an expert group on ethical, legal and social aspects of genetic testing. The focus will be on the issue of genetic exceptionalism, the place of genetic information in public health regulations and how direct to consumer offers on internet may challenge the existing regulations. This is of particular relevance as regard to the French situation where the law that regulates genetic testing is a 'bioethics law' that is under revision in 2009 .

PRENATAL FETAL RH D TYPING IN MATERNAL BLOOD [218]

S.J.H. Cheong and M.J. Sinosich

Molecular Genetics, Sonic Clinical Institute, Macquarie Park, Australia

Determination of fetal RHD genotype using a non-invasive approach is an important challenge with crucial clinical implications in management of pregnant RHD-negative women. We describe a single tube multiplex realtime (RT) - PCR assay for RHD fetal genotyping, adapted from a published report (Finning et al., 2002). Our study group consisted of normal non-pregnant $(n=80)$ and normal pregnant women $(n=37$ : Gestation $=9-11$ completed weeks). One (1) mL of maternal EDTA plasma was used for DNA (or RNA) extraction. Within this gestation window, there was no correlation between either maternal weight or gestation to concentration of fetal DNA. In control (non-pregnant population), RHD typing and gender assignment demonstrated $100 \%$ specificity. For confirmation of a female fetus (negative SRY signal), extracted RNA was analysed for presence of a fetoplacental-derived message. Male fetuses were correctly identified in 18 of 19 pregnancies (the 19th pregnancy was tested before inclusion of fetoplacental marker). Of the 6 RHD-negative mothers, five (5) were carrying RHD-positive babies. In this report we conclude that in first trimester of pregnancy, extracellular fetal DNA was readily detected, at significant concentration, to enable accurate fetal sexing and RHD genotyping.

\section{AN AUSTRALIAN TSC COHORT: ARE SURVEILLANCE GUIDELINES BEING MET? [195]}

M. Chopra', J. Lawson², M.Wilson ${ }^{3}$, S. Kennedy ${ }^{4}$, O.Wargon ${ }^{5}$, J. Dzarir ${ }^{6}$, P.Taylor ${ }^{6}$, and D. Mowat

Departments of ${ }^{\prime}$ Clinical Genetics, ${ }^{2}$ Neurology, ${ }^{4}$ Nephrology, ${ }^{5}$ Dermatology, Sydney Children's Hospital; ${ }^{3}$ Department of Clinical Genetics, Children's Hospital at Westmead; ${ }^{6}$ Molecular and Cytogenetics Unit, Department of Haematology and Genetics, SEALS, New South Wales, Australia

The aims of this study were to describe the clinical and radiological features of 45 Australian patients with Tuberous Sclerosis Complex (TSC), to assess enotype-phenotype correlation and to evaluate adherence to surveillance guidelines. Children and adults who fulfilled criteria for a diagnosis of TSC were recruited. TSC1/TSC2 gene sequencing and MLPA were performed. Clinical and radiological features and recent surveillance were recorded. There were 45 patients ( 22 males, 23 females) of whom 31 were children (age 0-17) and 14 adults (age > 18). 39 patients had de novo TSC, 5 were familial and 1 was uncertain. There were $9(20 \%)$ patients with TSC1 mutations, $22(49 \%)$ with TSC2 mutations, 4 (9\%) unclassified variants (UCV), and in 10 patients $(22 \%)$ no mutation was identified (NMI). $90 \%$ of patients had hypomelanotic macules, $78 \%$ of patients had seizures, $60 \%$ developmental delay and $32 \%$ autistic spectrum disorder. Cortical tubers were present in $88 \%$ of patients, renal cysts in $37 \%$ and angiomyolipomata in $39 \%$. 10/22 (45\%) patients with TSC2 mutations had autistic spectrum disorder (ASD), compared with 1/9 (11\%) TSC1 patients and $1 / 10(10 \%)$ NMI patients $(p<.05)$. While trends suggesting increased severity with TSC 2 mutations for other clinical features were 
observed, these did not reach statistical significance. 33/45 (73\%) patients were undergoing recommended surveillance. $25 / 28(89 \%)$ of pediatric patients met surveillance guidelines, compared with $5 / 14(36 \%)$ adult patients $(p<.05)$. The mutation and phenotypic spectrum is consistent with previous studies. Patients with TSC2 mutations had a significantly increased risk of ASD compared with those who had TSC 1 mutations and those who were mutation negative. Most adults in our study were not meeting surveillance guidelines.

\section{DOES SIZE MATTER?: LIKELIHOOD OF FINDING CAUSATION IN POSSIBLE XLMR FAMILIES INCREASES MULTIGENERATIONAL AFFECTED MALES [200]}

\section{Christie, M. Field, G. Turner, J. Boyle, C. Rogers, C. A. Turner, and A. Hackett} Genetics of Learning Disability, Hunter Genetics Newcastle, Australia

X-Linked Mental Retardation (XLMR) affects one in 650 males. More than 80 genes on the $\mathrm{X}$ chromosome that control cognition have been identified. The NSW GOLD service provides a tertiary level clinical service to XLMR families and offers participation in international collaborative research to determine diagnosis. Testing of probands may include X-tiling path array, sequencing of coding exons on the $\mathrm{X}$ chromosome and/or targeting specific mutations in known XLMR genes. The GOLD service currently has over 250 nonfragile $X$ families with a probable XLMR mode of inheritance, ranging from brother or nephew/uncle pairs to large multigeneration pedigrees. $46 \%$ of families have two or more affected males across generations with an obligate female carrier $54 \%$ of families have $2-5$ affected brothers in a sibship. Our overall definite diagnostic rate is $21 \%$. A definite diagnosis was obtained in $35 \%$ of multigenerational families compared with $10 \%$ (significant at $p=.04$ ) of male sibship families. There is a nonsignificant trend for larger families within these groups to have a diagnosis. For example, $39 \%$ of XLMR families with $>=4$ affected males have a definite diagnosis compared to $29 \%$ for XLMR families with 2-3 affected males. The presence of intellectual disability across generations is a better predictor of X-linked inheritance than number of affected males. Our figures are consistent with XLMR accounting for $\sim$ one-third of male sibship families.

\section{PROGRESS IN THE HUMAN VARIOME PROJECT [142]}

\section{R. G. H. Cotton and Collaborators}

Convenor Human Variome Project: Head, Genomic Disorders Research Centre, Howard Florey Institute; Department of Medicine, the University of Melbourne, Australia

The Human Variome Project (Axton, 2007) (www.humanvariomeproject. org) was initiated in June 2006 (Ring, Kwak et al., 2006) drawing attention to the importance of collection of variation and its phenotype and to develop programs to put this into effect.

The project builds on work and concepts of the HGVS over many years (www.hgvs.org) to focus on all variation associated with disease. The project will include those discovering mutations, their effects and then collect the data making it instantly available for those who need it to inform clinical decisions and research. The HVP and InSiGHT (www. insight-group.org) has developed a major pilot study to develop procedures and systems to allow effortless flow of de-identified data from the patient/ clinic/diagnostic laboratory via curated locus or gene specific databases to central databases/genome browsers such as NCBI, UCSC and EBI. The system will be easily adaptable to other genes and to multiple laboratories, states and countries worldwide. A country specific collection pilot is underway in Australia and an International Confederation of these countries has been initiated with Korea and China in the application process. Other pilot studies developed include specific ethical studies related to mutation collection, loading of LSDB content to dbSNP, funding of curation of LSDBs, a system of Microattribution/reward for mutation submission and a high level meeting in Spain (Kaput et al., In press) developed plans to implement the recommendations of the HVP Melbourne meeting (Cotton, Appelbe et al., 2007). The HVP was features recently in relation to Neurogenetic databases (Cotto, Auerbach et al., 2008).

\section{COMPARISON OF THE DIFFERENT B-THALASSAEMIA CARRIER SCREENING PROGRAMS CONDUCTED AROUND THE WORLD [28]}

N. Cousens', M. Delatycki',2, and C. Gaff ${ }^{2}$

'Murdoch Children's Research Institute, Australia

2 Genetic Health Services Victoria Melbourne, Australia

Beta-thalassaemia is an autosomal recessive condition, caused by mutations in the $\beta$-globin gene, resulting in individuals having chronic anaemia. The management of this condition involves regular blood transfusions and iron chelation therapy. Thalassaemia carrier screening programs have been developed in Southeast Asia, India, the Middle East, Mediterranean and European countries in order to reduce the incidence of this condition. The aim of this study is to compare the $\beta$-thalassaemia carrier screening pro- grams around the world in order to further understand the variations in the structure of the different programs. A systematic analysis of articles describing the structure of $\beta$-thalassaemia carrier screening programs was conducted using the Medline and Pubmed databases. Twenty-three published journal articles were found, describing 16 different $\beta$-thalassaemia carrier-screening programs. Programs in the following countries have been described: Iran, Saudi Arabia, Gaza Strip, Turkey, Greece, Cyprus, Italy, Canada, UK, China, Taiwan, India, France and The Maldives. A great variation is seen among the different programs. Variations found between the programs include mandatory vs. voluntary screening; antenatal vs. preconceptional screening; age at which screening is offered; individuals giving informed consent for screening vs. individuals unaware of screening taking place; and education and health promotion provided in numerous ways vs. no education in place at all. Different $\beta$-thalassaemia carrier screening programs across the world vary in numerous ways. Every country has different needs due to differences in prevalence, culture, religion, financial circumstance and law.

\section{ATTITUDES TOWARDS NEWBORN SCREENING FOR POMPE DISEASE [79]}

Y. Curlis ${ }^{1,2}$, L. Curnow ${ }^{1,2}$, and H. Peters ${ }^{1,2}$

' Department of Paediatrics, The University of Melbourne, Australia

${ }^{2}$ Genetic Health Services Victoria, Murdoch Children's Research Institute, Australia

Pompe disease is a rare, progressive and often fatal muscular disease caused by a deficiency in lysosomal alpha glucosidase. Two broad clinical categories of Pompe disease have been identified; infantile and late onset. Treatment using enzyme replacement therapy has shown promising results. With the recognition that early treatment leads to a better outcome, newborn screening (NBS) for Pompe disease has been proposed. Ninetyseven adults with Pompe disease from Australia, the United Kingdom and United States and one hundred Victorian parents of 'healthy' children completed questionnaires exploring their experiences of Pompe disease and/or NBS and attitudes towards NBS for Pompe disease. Support for NBS for Pompe disease was high among both adults with Pompe disease $(85.4 \%)$ and parents of healthy children $(93.9 \%)$. When offered a theoretical screening test that would only identify juvenile onset Pompe disease, $42.1 \%$ of adults with Pompe disease and $53.1 \%$ of parents of healthy children stated that they would prefer this screen. Factors influencing attitudes were the participant's age of symptom onset and a belief that an earlier diagnosis would have made symptoms easier to deal with. Participants who supported newborn screening for Pompe disease were more likely to have wanted to have been diagnosed through NBS and did not prefer the theoretical screen.

\section{PRENATAL DETECTION OF AN UNUSUAL RECOMBINANT CHROMOSOME (3) RESULTING FROM A MATERNAL COMPLEX CHROMOSOME REARRANGEMENT [132]}

P. Dalzell', F. Xu', S. Byatt', B. Miller', D. Mowat ${ }^{2}$, D. Challis ${ }^{3}$, and M.-Y.Yip' 'Molecular \& Cytogenetics Unit, SEALS, Prince of Wales Hospital, Sydney, Australia 2 Department of Medical Genetics, Sydney Children's Hospital, Sydney, Australia Maternal Fetal Medicine, Royal Hospital for Women, Sydney, Australia

Complex chromosome rearrangements (CCR) can be defined as structural rearrangements with more than two breakpoints involving exchange of genetic material between two or more chromosomes and their prevalence is extremely rare. Cytogenetic analysis was performed on a fetal pleural effusion obtained from a 32 week gestation pregnancy and showed an apparent insertion of unidentified material into the short arm of chromosome 3. Maternal peripheral blood analysis revealed a CCR with both an insertion and a translocation. As the result of crossing over involving the maternal derivative chromosome 3 , the fetal karyotype is $46, \mathrm{XY}, \operatorname{rec}(3)$ $\operatorname{dup}(11 \mathrm{q}) \operatorname{ins}(3 ; 11)(\mathrm{p} 23 ; \mathrm{q} 13.5 \mathrm{q} 21), \mathrm{t}(3 ; 11)(\mathrm{q} 13.2 ; \mathrm{q} 21)$ mat. The resultant dup(11)(q13q21) was confirmed by both Fluorescence in situ hybridisation (FISH) and array CGH analysis.

\section{THE IMPACT OF THE METASYSTEMS METAFER SCANNING SYSTEM ON THE ONCOLOGY SERVICE, GENETIC SERVICES, WELLINGTON [101]}

\section{G. Davis, D. Farrell and C. Felix}

Central and Southern Regional Genetic Services, Wellington, New Zealand

The Metasystems Metafer metaphase finder was introduced into the Genetic Services Laboratory, Wellington Hospital in April 2007 for all oncology samples. An audit of data 12 months prior and 12 months post implementation was undertaken to assess the impact of this system upon our oncology cytogenetic service. The oncology section processed a total of 1592 samples during this period including bone marrows, leukaemic bloods, lymph nodes and solid tumors. Our data demonstrates that the scanning system has had a profound affect on our service with regard to reporting times, success rates and abnormality rates. Representative 
cases will be presented to support these data. An outline of sample workflow and quality control procedures introduced at implementation will also be discussed.

\section{CADASIL: UPDATING THE TESTING PARADIGM [157]}

M. Davis, R. Gooding, and P. Lamont

Neurogenetics Unit, Department of Anatomical Pathology, Royal Perth Hospital, Perth Australia

Cerebral autosomal dominant arteriopathy with subcortical infarcts and leucoencephalopthy (CADASIL) is an inherited condition characterized by migraine and adult-onset recurrent stroke leading to progressive cognitive impairment and premature death. The disease is caused by mutations in the NOTCH 3 gene, which encodes a transmembrane receptor involved in cell fate specification during development. The gene is large, with 33 exons encoding a protein of 2321 amino acids. The extracellular part of the protein contains 34 epidermal growth factor-like domains, and it is in these domains, encoded by the first 23 exons of the gene, that mutations occur. Early studies showed that exons 3 and 4 accounted for the majority of mutations, with $81 \%$ occurring in these exons in the British population. On the basis of this data, the initial screening protocol developed by this and other laboratories was confined to these exons. More recent studies have however highlighted that different populations have different mutation hotspots. We therefore expanded the panel of exons screened to include 3-8 and 11, and as a consequence our mutation detection rate has increased by $60 \%$. This study shows that care must be taken when making presumptions about likely testing results based on the ethnic background of the patient population, and demonstrates the necessity of periodic review of molecular testing protocols in the light of newly published data.

\section{A RARE CASE OF T(12;22)(P13;012) IN ACUTE MYELOID}

\section{LEUKAEMIA [171]}

R. de Kraa', S. Clarke', J. O'Reilly', B.Augustson ${ }^{2}$, J. Peverall', and F. Price ${ }^{3}$

Department of Haematology, Royal Perth Hospital, Perth, Australia

Western Diagnostic Pathology, Australia

King Edward Memorial Hospital for Women, Australia

A 77-year-old female, presented with a mild monocytosis and thrombocytopaenia for further investigation. The full blood picture showed a $\mathrm{Hb}$ of $128 \mathrm{~g} / \mathrm{L}$, a WCC of $12.2 \times 10^{\wedge} 9 / \mathrm{L}$, aplatelet count of $100 \times 10^{\wedge} 9 / \mathrm{L}$ and a mild monocytosis of $2.1 \times 10^{\wedge} 9 / \mathrm{L}$. The bone marrow findings were consistent with acute myeloid leukaemia, the features highly suggestive of transformed chronic myelomonocytic leukaemia. Cytogenetic analysis revealed the karyotype: 46,XX,t(12;22)(p13.?1;q12.?1)[20] FISH analysis using the Vysis ETV6 probe for chromosome 12p13 and an in-house EWSR1 probe for chromosomes 22q12.2 confirmed a reciprocal translocation between chromosomes 12 and 22 at bands p13 and q12.2 respectively. The $\mathrm{t}(12 ; 22)$ is an infrequent rearrangement in myeloid malignancies. Both the 22q11 and 22q12 breakpoints have been described, involving MN1 and EWSR1 genes respectively. This case study described the rare finding of $\mathrm{t}(12 ; 22)$ in this patient with acute myeloid leukaemia.

\section{EIGHT MICRO ARRAY PLATFORMS COMPARED FOR CLINICAL CYTOGENETIC USE [17]}

D. de Lagarde', S. Sivamoorthy', M. Di Renzo', A. Murch', A. Darmanian²,

G. Peters'², J. Hui', M. Jennens', G. Arscott', J. Beilby', S.Wilton ${ }^{3}$, T. Ly',

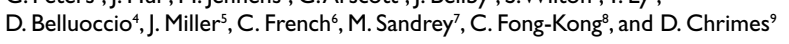
'PathWest

$\mathrm{CHW}$

ANRI

$P L P$

Affmetrix

Roche

Illumin

${ }^{8}$ PerkinElmer

BlueGnome Ltd

In recent studies, microarray analysis of copy number variants $(\mathrm{CNV})$ in genomic DNA from selected patients with mental retardation and/or dysmorphic features who are karyotypically normal by conventional cytogenetic analysis, detects pathologically significant CNV in $10 \%$ or more of cases. Currently, microarray is in limited clinical use for a number of reasons, one of which is the wide range in platforms available and rapid development of both hardware and software available for analysis. For this reason we decided to trial several vendor platforms to determine which system best suited our needs: reliable results, affordability, easy to follow protocols, user friendly software, limited user variability, quick analysis that produces understandable reports for the requesting physicians and dependable technical support. In this investigation we trialled the following eight different microarray platforms; The PerkinElmer Constitutional 3.0 Chip and Spectral Chip 2600, the BlueGnome Cytochip v2, the Agilent $4 \times 44 \mathrm{~K}$ CGH microarray, the Illumina $370 \mathrm{CNV}$ quad chip and
Human Cyto12SNP array, the NimbleGen 4x72k array and the Affymetrix Cytochip. One patient with a 14q12 deletion indentified by Cytogenetics will be showcased. The comparison will consists of platform differences, results generated from each vendor, software differences, and the pros and cons we experienced for each system.

\section{SUCCESSFUL PREGNANCY AND DELIVERY IN A YOUNG WOMAN WITH POMPE DISEASE [126]}

K. Devine', B. Trudinger' ${ }^{2}$ T. Robinson ${ }^{3}$, S.Vucic ${ }^{4}$, M.Tchan', and D. Sillence'

Departments of Genetic Medicine $e^{l}$, Obstetrics and Gynaecology ${ }^{2}$, Respiratory Medicine ${ }^{3}$ and Neurology, Westmead Hospital, Sydney, Australia

Pompe disease is an insidious progressive muscle disorder resulting from a deficiency in lysosomal acid alfa-glucosidase. The late-onset form of the disorder is characterised by progressive limb girdle weakness and respiratory insufficiency. We report a successful pregnancy and delivery in a 32 -year-old woman with late-onset Pompe disease. This young woman initially had symptoms of fatigue and exertional dyspnoea from 16 years of age. Diagnosis was made at 24 years on the basis of a muscle biopsy showing acid alfa-glucosidase activity at $14.8 \mathrm{pmol} / \mathrm{min} / \mathrm{mg}$ (NR 67-1300) and a blood spot analysis showing undetectable alfa-glucosidase activity. Over the past decade she has had progressive weakness and by the time she became pregnant she was unable to run or climb stairs and had considerable difficulty standing from a sitting position. Lower limb strength grades were - hip flexion and abduction $3+/ 5$ and hip extension $2 / 5$ bilaterally. Over the past few years she has also had progressive dyspnoea to the point where she commenced nocturnal non-invasive ventilation (VPAP) at age 31 . Her pre-pregnancy pulmonary function test showed a significant fall in FVC in the supine position - compatible with significant diaphragmatic weakness. Her pathology at the time showed a CK of $842 \mathrm{U} / \mathrm{L}$ (NR 0-180). In the early stages of the pregnancy she showed some signs of weakening but this did not progress. She presented in labour at term and had a forceps assisted delivery. A female infant weighing $3380 \mathrm{gm}$ was delivered. Mother and baby were discharged home 3 days later.

\section{THE GENETICS OF SPINAL MUSCULAR ATROPHY: IMPLICATIONS FOR POPULATION SCREENING AND CLINICAL MANAGEMENT OF FAMILIES [151]}

\section{D. du Sart', M. Smith', B. Chong', M. Kaps', and A. Kornberg'}

Molecular Genetics Laboratory, VCGS Pathology, Australia

Royal Children's Hospital, Murdoch Childrens Research Institute, Melbourne, Australia

Spinal Muscular Atrophy (SMA) is the second most common severe recessive disorder after cystic fibrosis and the most frequent genetic cause of infant mortality. It is characterized by degeneration of the anterior horn cells of the spinal cord, leading to symmetrical muscle weakness and atrophy. The SMA determining gene is named the survival motor neuron 1 (SMN1) gene, which has $\sim 98 \%$ homology with a pseudogene, SMN2. SMA has an estimated incidence of 1/10,000 live births with a high carrier frequency of $1 / 40$ in the general population. The copy number of SMN1 varies greatly in different individuals and clinical severity is mediated by copy number of SMN2. Most affected individuals have no copies of SMN1, most carriers have one copy of SMN1 and non-carriers can have two to four copies of SMN1; SMN2 copy numbers can vary from 0 to $>5$. Identification of SMA carriers has important implications for individuals. SMA carriers are completely healthy and most are unaware of their carrier status until they have an affected child. Provision of a fully comprehensive DNA and RNA testing service for SMA over the past 7 years, has gathered a wealth of data which hi-lights the complexities of the SMN region, the unexpectedly high rate of de-novo deletions and point mutations, gene conversion events and placental mosaicism events that impact on prenatal diagnoses and PGD services. Details of these events and a review of the current knowledge of SMA, including current available clinical trials, will be presented.

\section{CHALLENGES IN GENETIC TESTING OF CARDIAC ARRHYTHMIA DISORDERS — BEST PRACTICE GUIDELINES FOR LQT TESTING [153]}

\section{D. du Sart}

Molecular Genetics Laboratory, VCGS Pathology, Murdoch Childrens Research Institute, Melbourne, Australia

Inherited cardiac arrhythmias, particularly Long QT syndrome (LQT), which involve abnormal electrical activity in a structurally normal heart, are conditions that cause unexpected sudden death occurring during physical activity, at times of emotional stress or even whilst asleep. The estimated prevalence is 1 in 3000; usually autosomal dominant inheritance and the symptoms include fainting, epilepsy and sudden death, particularly in young people. Genetic testing for these disorders can identify individuals and their relatives who have the condition with greater precision than routine cardiac testing. Affected individuals need lifelong 
treatment and unaffected individuals can be freed from unnecessary follow-up testing or inappropriate medication, typically beta-blockers, and sometimes implanted devices to reduce risk of sudden death. Genetic testing for LQT involves screening of 6 genes that are involved in forming the membrane ion channels in cells. There are huge issues about the level of testing, defining which are the pathogenic gene changes and the next level of complexity, in terms of managing and reporting test results, and then dealing with the issues in a clinical setting, how families deal with this info with uncertain clinical significance.

\section{THE HUMAN VARIOME PROJECT AND THE DIAGNOSTIC LABORATORY: THE IDEAL SYSTEM [154]}

D. du Sart,V. Hyland, J. MacMillan, F. Macrae and R.G.H. Cotton and many representatives of all states

One of the most significant outcomes from the Human Genome project is the translation of the genome data into clinical practice. This information impacts on clinical diagnosis of affected patients and clinical management of the at-risk family members. Molecular Genetic Diagnostic Laboratories are involved in this process of testing affected patients and at-risk family members. Consequently, these laboratories collect a huge amount of data about the genes that they analyze in many different patients. This is data about sequence variation present in the human genome and it is usually linked to a clinical phenotype. One of the vital aims of the Human Variome Project is to facilitate the collection of gene variation data and related clinical information to a central data collection centre that is managed in the appropriate ethical and confidential manner. Why should this be done? Diagnostic laboratories need to interpret the significance of sequencing data obtained when screening genes in patients. They rely heavily on databases to provide information on whether a specific sequence change has been identified before, whether the change is part of normal variation, what is the frequency of the change. But, are these databases regularly updated with all available information? And how do laboratories benchmark their interpretation of results in terms of the clinical significance for patients and families? This presentation will outline the value of the Human Variome project to provide benchmarking and consistency in result interpretation within the Australian Laboratories.

\section{HLA ASSOCIATED DISEASE TESTING: COELIAC DISEASE [173]}

A. Duffy', H. Clague', A. Pink', D. Heyworth-Smith², D. Gillis ${ }^{3}$, and P. Hogan ${ }^{4}$ ' Class II Tissue Typing Laboratory, Division of Immunology, Pathology QueenslandCentral Laboratory, RBWH, Herston, Australia

${ }^{2}$ Consultant Clinical Immunologist, QML Pathology, Brisbane Laboratory, Australia ${ }^{3}$ Senior Staff Specialist, Division of Immunology, Pathology Queensland, RBWH, Herston, Australia

${ }^{4}$ Director of Immunology, Pathology Queensland, RBWH, Herston, Australia

Coeliac disease is a chronic inflammatory disorder of the gastrointestinal tract that is induced by exposure to an environmental precipitant, gluten, in the diet of susceptible individuals. Although coeliac disease is reasonably common (estimated frequency of $1 \%$ ), many other patients have noncoeliac wheat intolerance. These patients are not infrequently on gluten free diets without a definite diagnosis of coeliac disease and are subsequently shown not to have coeliac disease. Conversely, many patients with coeliac disease have commenced a gluten free diet prior to serological or biopsy diagnosis. Although a small bowel biopsy provides the definitive diagnosis of coeliac disease, $>95 \%$ of sufferers have either the HLA-DQ2 (DQA $1 * 0501 / \mathrm{DQB} 1 * 0201$ or DQA $1 * 0501 / \mathrm{DQB} 1 * 0202)$ or the HLADQ8 (DQA $1 * 0301 / \mathrm{DQB} 1 * 0302$ ) genotype. Coeliac disease rarely occurs in patients who do not have this genotype and these patients can have gluten introduced back into their diet without onset of coeliac disease. Thus HLA genetic testing has been increasingly used to help exclude coeliac disease in patients with suggestive symptoms. Molecular testing may be particularly helpful when patients are reluctant to resume a gluten inclusive diet to facilitate nongenetic diagnosis. Because of the increasing demand for HLA testing for coeliac disease, our laboratory developed an in-house sequence-specific PCR test for the disease-associated alleles. In 2008 we processed over 400 tests, of which approximately $60 \%$ were positive for at least one disease-associated haplotype. This study involves a retrospective analysis of the clinical features, coeliac serology profiles and ultimate diagnosis of patients who were referred for molecular testing over one year.

\section{A RARE CASE OF T(6;11) IN AML [204]}

N. Egan and J. O'Reilly

Department of Haematology, Royal Perth Hospital, Australia

Rearrangements of the MLL gene located at 11q23 are nonrandom abnormalities seen in 5-6\% of cases with acute myeloid leukaemia (AML), usually with monocytic features. MLL gene rearrangements involve a large number of genetic partners. The most common MLL translocation in AML is $\mathrm{t}(9 ; 11)(\mathrm{p} 22 ; \mathrm{q} 23)$, seen in $2.2 \%$ of adult patients with de novo
AML. A less common finding is $\mathrm{t}(6 ; 11)(\mathrm{q} 27 ; \mathrm{q} 23)$ involving the AF6 gene that has been identified in $0.6 \%$ of AML patients. A recent study of patients with $\mathrm{t}(6 ; 11)$ found this to be associated with a poor prognosis when compared to the standard risk group for AML. We present a case study of a 26-year-old female who presented in October 2008 with $73 \%$ blasts in her peripheral blood. A bone marrow aspirate was performed and she was subsequently diagnosed with acute myeloid leukaemia with M5a morphology. Cytogenetic analysis revealed $t(6 ; 11)(q 27 ; q 23)$ and FISH studies confirmed involvement of the MLL gene. These results are consistent with the WHO classification: AML with 11q23 (MLL) abnormalities and a morphological association with M4/M5. This patient is now in complete remission following her first cycle of consolidation chemotherapy.

\section{MLPA ANALYSIS OF 145 AUTISM SPECTRUM PATIENTS [158]}

\section{Field and $\mathrm{H}$. Puusepp}

GOLD Service, Hunter Genetics, Australia

Two genomic copy number changes, dup 15q11-13, and del 16p11.2 have been shown to occur in patients with an autistic spectrum disorder (ASD) with a frequency of at least $1 \%$. Other recurrent copy number variants including del 22q13.3, dup 17p11.2 and dup 16p13.1 also are reported in the ASD population. Multiplex Ligation-dependent Probe Amplification (MLPA) is a widely used technique for targeted screening of specific copy number variations because of its accuracy, robustness, low reaction costs and the relative ease with which probes can be generated. We have used commercial SALSA MLPA P245 microdeletion syndromes probemix, with an additional eight designed probes to regions $16 \mathrm{p} 11.2$ and 16p13.1.DNA from saliva and whole blood were analyzed from 145 patients with ASD. Seven of 145 patients $(4.8 \%)$ have a possible disease-related imbalance including a possible mosaic sex chromosome aneuploidy, two dup $22 q 13.33$, del 5q12, del 16p11.2, dup16p13.1 and dup15q11-13. Confirmation of these findings using alternate techniques is underway. Benefits of the technique include low cost, possible avoidance of need for a blood collection, and the ability to detect small genomic imbalance known to be specifically associated with an autistic spectrum disorder.

\section{WHO OWNS THE PAST? THE CIRCULATION OF BIOVALUE ACROSS SPACE AND TIME [224]}

\section{A. Fletcher}

University of Canterbury, New Zealand

This paper asks: what institutions and norms are emerging to regulate the extraction of biovalue from ancient sources, and what are the implications for health innovation and research? The concept of biovalue provides a powerful tool for analysing the political economy of emerging biotechnologies, as it extends Marxist theory to a critique of the exploitation of the 'surplus vitality' of genes. This approach is increasingly used to examine new institutions such as national human gene and tissue banks, in which genetic and medical information gathered from individuals and cultural groups translates into private gain via the development of patented products. This paper extends the notion of biovalue from its present emphasis on the circulation of genetic information through space to an analysis of its circulation through time. Recent research on mammalian cloning via tissue samples sourced from long-dead (and frozen) animals, and possibly even from extinct species, provides another potentially limitless source of biovalue to be extracted in the construction of a global bio-economy. Yet such research also smudges the line between life and death, implicitly altering our relationship to time, and explicitly challenging established individual and cultural narratives. Via a comparison of emerging ancient DNA research projects to the previous international controversy surrounding the Icelandic Health Sector Database, this paper explores the biopolitical implications of mining the past in the pursuit of future health.

\section{INDUCED NONPRODUCTIVE SPLICING TO STUDY} MUSCLE GENE EXPRESSION [150]

\section{S. Fletcher,A.Adams, R. Johnsen, K. Greer, and S.Wilton}

Molecular Genetic therapy Group, CNND, University of Western Australia, Perth

RNA silencing has been applied to suppress gene expression, with varying degrees of specificity and efficiency reported. Endogenous alternative splicing can regulate gene expression through a process called Regulated Unproductive Splicing and Translation (RUST) by either incorporating an exon carrying a nonsense mutation, or excluding an exon to disrupt the reading frame. As a result, the mature gene transcripts cannot be translated into functional gene products. We show that it is possible to efficiently disrupt the normal dystrophin mRNA reading frame and ablate dystrophin expression by the application of antisense oligomers. Total suppression of dystrophin gene expression can be induced and maintained for several weeks in vivo, and a severe dystrophic pathology observed within 4 weeks of commencing oligomer delivery in neonatal mice. This approach to gene down-regulation is very efficient and specific in suppressing dystrophin gene expression, unlike a recent attempt to induce 
dystrophic pathology by RNAi. Disruption of gene expression through altered splicing patterns could be applied to many different genes, and offers the opportunity to induce transient mouse models to study the consequences of gene suppression in vivo. In addition, selected exon removal to yield in-frame transcripts can allow mapping of functional protein domains, based upon exon boundaries, and provide an alternative to transgenic mouse models for the study of muscle gene expression.

\section{THE UPTAKE OF GENETIC TESTING BY AT-RISK FAMILY MEMBERS: AN AUDIT OF CLINICAL SERVICE [148]}

\section{E Forrest ${ }^{1,2,3}$, M. B Delatycki $i^{2,3,4}$, L. Curnow ${ }^{4}$, L. Skene ${ }^{5}$, and M. Aitken ${ }^{6}$}

Genetics Education and Health Research, Murdoch Childrens Research Institute,

10th Floor, Royal Children's Hospital, Australia

Department of Paediatrics, The University of Melbourne, Australia

Bruce Lefroy Centre for Genetic Health Research, Murdoch Childrens Research Institute, 10th Floor, Royal Children's Hospital, Australia

Genetic Health Services Victoria, Royal Children's Hospital, Australia

Melbourne Law School, The University of Melbourne, Australia

Research Strategy, Murdoch Childrens Research Institute, Royal Children's Hospital, Parkville, Victoria, Australia

Communicating information about a genetic condition in families provides at-risk family members with the opportunity to make decisions about genetic testing. Those who choose to have genetic testing indicate definitively that family communication has occurred. In Victoria, carrier testing for fragile X syndrome (FXS) and Spinal Muscular Atrophy (SMA), and predictive testing for Huntington disease (HD), is conducted by a single state-wide laboratory. We conducted a clinical audit using genetics files from Genetic Health Services Victoria and linked the data to the laboratory reports of the Victorian Clinical Genetics Service to determine the percentage of at-risk family members who had genetic testing. The proband in each family was diagnosed with FXS, SMA or HD in a 5year period between the 1st January 2000 and the 31st December 2004. The raw percentage of family members who had carrier testing for FXS was $16 \%(43 / 264)$, predictive testing for HD was $17 \%(28 / 164)$, or carrier testing for SMA was $11 \%(33 / 290)$. First-degree at-risk family members were the most frequently tested group, and the frequency of testing declined with relatedness. Of all family members who had genetic testing, $86 \%$ (81/94) had done so within two years of the proband's diagnosis. These results demonstrate that the majority of at-risk family members do not have genetic testing. Family communication is obviously occurring, nevertheless, this method of communication may not be assumed to result in family members who understand the personal implications of the condition in order to make an informed decision about genetic testing.

\section{COMMUNICATING GENETIC INFORMATION IN FAMILIES: HOW WELL INFORMED ARE FAMILY MEMBERS? [149]}

\author{
L. E Forrest ${ }^{1,2,3}$, M. B Delatycki $i^{2,3,4}$, L. Curnow ${ }^{4}$, L. Skene ${ }^{5}$, and M.Aitken ${ }^{6}$ \\ Genetics Education and Health Research, Murdoch Childrens Research Institute, Royal \\ Children's Hospital, Parkville, Australia \\ Department of Paediatrics, The University of Melbourne, Australia \\ Bruce Lefroy Centre for Genetic Health Research, Murdoch Childrens Research Institute, \\ Australia \\ Genetic Health Services Victoria, Australia \\ Melbourne Law School, The University of Melbourne, Australia \\ Research Strategy, Murdoch Childrens Research Institute, Royal Children's Hospital, \\ Parkville, Australia
}

A dichotomy exists in the literature about informing family members that they are at risk of a genetic condition. One aspect focuses on nondisclosure of genetic information in families and the ethical ramifications of this situation. The alternative to nondisclosure, and often the default position, is communication. While nondisclosure is likely to occur less frequently than communication, it has received considerably more attention particularly regarding action genetic health professionals should take to warn uniformed at-risk family members. In contrast, communicating genetic information in families creates apparently few ethical or legally challenging situations for genetic health professionals as at-risk family members are assumed to be informed. We investigated families' communication of genetic information using a three stage mixed methodology study. This included (1) qualitative interviews with consultands and family members about their experience of communicating genetic information, (2) an online survey of genetic health professionals' practice involving family communication, and (3) a clinical audit of genetics files to determine the uptake of genetic testing by at-risk family members. The results from this study indicate that family communication cannot be assumed to result in informed family members. The process of communicating genetic information does not guarantee that family members understand the information, and the personal and reproductive implications that the genetic condition carries. This therefore inhibits their ability to make an informed decision about their risk. Therefore attention needs to be refocused on the role of genetic health professionals supporting communication of information to enable informed decision making by at-risk family members.

\section{SPINOCEREBELLAR ATAXIA MASQUERADING AS AN} INBORN ERROR OF METABOLISM IN AN INFANT [160]

\section{Gabbett and B. Holland}

Genetic Health Queensland, Australia

Spinocerebellar ataxia type 7 (SCA7) is caused by an expanded trinucleotide repeat in the ATXN7 gene. The hallmark features of SCA7 are progressive cerebellar ataxia and a pigmentary macular degeneration. However large trinucleotide expansions in ATXN7 can present as a very different and rare clinical phenotype. We report on a 2-year-old girl with a suspected inborn error of metabolism who died after an illness characterised by progressive hypotonia and profound nephrosis. Her half brother was diagnosed with SCA7 six years after her death. It was subsequently apparent that the two year old died from very rare complications of SCA7. We present this case to illustrate that SCA7 may present as progressive hypotonia and nephrotic disease in infancy.

\section{A LIFE WORTH LIVING: DECISION-MAKING IN THE CONTEXT OF PRENATAL DIAGNOSIS FOR AN INHERITED CANCER SYNDROME [58]}

\section{Gaffney and L. Gordon}

Genetic Health Services Victoria, Australia

Tim* (37 years) and Kate* (34 years) were referred to a prenatal genetics service from a familial cancer centre to discuss their unexpected pregnancy. Tim had an inherited cancer syndrome and his father, sister and grandmother had all died as a result of the condition. He was being investigated for several possible cancers at the time of the referral. The familial cancer centre had requested mutation detection and results were pending. The couple attended for genetic counseling initially to discuss the pregnancy and the option of prenatal diagnosis. A mutation was detected in Tim and the couple attended again to discuss prenatal diagnosis in more detail. They chose to proceed with prenatal diagnosis with the intention of terminating the pregnancy if the fetus had inherited Tim's mutation. I will discuss the many challenges involved in counseling this couple and reflect upon the unique issues involved for clients and counselors working in similar cases, including: prenatal diagnosis for an adult onset condition; considering termination of pregnancy for a dominant condition, planned versus actual behavior and counseling an individual with a life threatening condition.

\section{EVALUATION OF DIAGNOSTIC GENETIC TESTS AND \\ COMPLEX BIOMARKERS [54]}

C. George, C.Wright, and R. Zimmern

Foundation for Genomics and Population Health (PHG Foundation), United Kingdom

There has been a massive expansion in research efforts to understand the genetic basis of disease. Reliable evidence of associations between genetic variants and the risk of developing specific complex (multifactorial) diseases are rapidly emerging. This new understanding has led to the development of many novel diagnostic, predictive and prognostic tests, to determine the presence (and, in some cases, likely course) of a specific disease, to predict the risk of developing disease in the future, or to predict tolerance and response to pharmaceutical treatments. The commercial market for direct-to-consumer tests is simultaneously burgeoning with genetic susceptibility tests that claim to predict the likelihood of an individual developing numerous complex diseases at some point in the future. Despite the increasingly crucial role of types of genetic test in clinical medicine and their growing availability directly the public, no formal evaluation or regulatory system exists to ensure the clinical effectiveness and utility of individual tests. The use of tests in the absence of proper evaluation could cause significant harm to unwary patients, while clinicians need guidance on which tests are valid and useful, and which are not. Pressure is now mounting to establish a formal evaluation framework for all clinical biomarker tests, including genetic tests, which is regulated in the most efficient and effective manner. The PHG Foundation is involved in both developing a coherent evaluation framework, through analysis of the conceptual issues involved, and driving national and international policy, through examination of the possible regulatory hurdles and solutions. The PHG Foundation has been working with national and international groups to consider the evaluation and regulation of clinical laboratory tests. In the $\mathrm{UK}$, in contrast to the position for pharmaceuticals, there is currently no requirement to provide evidence of clinical performance for diagnostic tests. 


\section{GENEALOGY FOR WESTERN AUSTRALIA -}

THE WA FAMILY CONNECTIONS PROJECT [203]

E. J. Glasson', A. J. Bass², N. H. de Klerk ${ }^{3}$, D. L. Rosman ${ }^{4}$, L. J. Palmer ${ }^{5}$

and C.D.J. Holman

School of Population Health, The University of Western Australia, Perth, Australia

Menzies Research Institute, University of Tasmania, Australia

Division of Population Sciences, Telethon Institute for Child Health Research, Australia Data Linkage Branch, Department of Health, Government of Western Australia, Perth, Australia

Centre for Genetic Epidemiology and Biostatistics, The University of Western Australia, Western Australia

The aim of the Western Australian (WA) Family Connections Genealogical project is to create and store a system of links representing genealogical relationships for the residents of WA to be used as a research tool in conjunction with health data to help study the inheritance of disease. It is supplementary to the WA Data Linkage System (WADLS) that coordinates regular linkages of records across several population-based health-related data sets. The genealogical links are made using information from birth, death and marriage registrations. Phase 1 of the project has involved creating genealogical links from information recorded on electronic birth registrations that are available since 1974 and electronic death and marriage registrations available since 1984 , totaling approximately 1.3 million records. Phase 2 will entail encoding genealogical links from earlier paper-based birth, death and marriage registrations, with an initial focus on the 0.9 million records available since 1950. Currently, the genealogy may support genetic and environmental epidemiological research projects for up to three-generational pedigrees. Ultimately, the data may be used to assess the degree of relatedness of individuals within study samples, assist in locating common ancestors and allow estimates of genetic risk. Population-based genealogies for extant populations are rare due to the challenges of developing and maintaining such a resource on a large scale. The combination of genealogy for the WA population and health data from the WADLS represent a unique opportunity to investigate the inheritance of human disease.

\section{CASK MUTATIONS CAUSE X-LINKED NYSTAGMUS AND}

\section{VARIABLE XLMR PHENOTYPES [223]}

A. Hackett', F. E Abidi' ${ }^{2}$ P.Tarpey ${ }^{3}$, A. Licata ${ }^{2}$,A.Whibley 4 , J. Boyle ${ }^{4}$, C. Rogers'

L. Christie', J. Grigg', M. Partington', R. E. Stevenson'2, G. Turner', M.Wilson ${ }^{6}$

C. E Schwartz ${ }^{2}$, J. Gecz', F. L. Raymond ${ }^{4}$, and M. R. Stratton ${ }^{3,8}$

' GOLD Service, Hunter Genetics, Waratah, New South Wales, Australia

JC Self Research Institute of Human Genetics, Greenwood Genetic Center, Greenwood,

South Carolina, United States of America

Wellcome Trust Sanger Institute, Hinxton, Cambridge, United Kingdom

${ }^{4}$ Cambridge Institute of Medical Research, Cambridge, United Kingdom

University of Sydney, Sydney Eye Hospital, Sydney, New South Wales, Australia

${ }^{6}$ Department of Clinical Genetics, Children's Hospital at Westmead, Australia

Department of Genetic Medicine, Women's and Children's Hospital, North Adelaide, Australia

${ }^{8}$ Cancer Research UK Genetic Epidemiology Unit, Strangeways Research Laboratory Cambridge, United Kingdom

The calcium/calmodulin-dependent serine protein kinase (CASK) gene has recently been identified as a novel gene for X-linked mental retardation (XLMR). Najm et al. (2008) identified CASK mutations in patients with microcephaly, optic atrophy and brainstem and cerebellar hypoplasia. We detected 4 unique missense changes in a cohort of XLMR probands (Tarpey et al. 2008). The affected males had a variable phenotype which included nystagmus. A fifth missense change has been identified in a female carrier from a small family, suspected of harbouring a CASK mutation based on her clinical phenotype of XLMR and nystagmus. Detailed clinical examinations, including an ophthalmological review in 3 families, were undertaken to further characterise the clinical phenotype associated with CASK mutations. Here we report on the clinical features of 19 individuals from 5 families. The phenotype was variable, ranging from nonspecific mild intellectual disability to severe mental retardation associated with microcephaly and dysmorphic facial features. Infantile nystagmus syndrome was present in three of the families. Carrier females were variably affected. Our findings reinforce the CASK gene as a relatively frequent cause of XLMR.

\section{TRANSLATING RESEARCH INTO PRACTICE AND PRACTICE INTO RESEARCH: RESEARCHERS' AND HEALTHCARE PROFESSIONALS' VIEWS OF CANCER GENETICS ACTIVITIES IN THE UNITED KINGDOM [228]}

\section{N. Hallowell}

University of Edinburgh, United Kingdom

The relationship between clinical research and clinical care is often perceived as unclear, particularly in highly technological subspecialties. This ambiguity is illustrated in cancer genetics where research protocols are frequently used to provide access to procedures that may be offered as a clinical service in other specialties. The project on which this presentation is based investigated lay and expert perceptions of the activities that take place within the cancer genetics clinic. This paper will focus upon the data collected in semi-structured interviews with individuals who are involved in cancer genetics research in the United Kingdom, in either a research only or research + clinical capacity. It will discuss the ways in which interviewees sought to distinguish research from clinical care and describe the strategies healthcare professionals said they used to differentiate these activities in their practice. It will be argued that distinguishing research from clinical care requires clinical researchers to engage in a form of 'boundary work' (Gieryn, 1999), and that this is necessary for them to manage the conflict of interest generated by the different roles they occupy within the cancer genetics clinic.

\section{MANAGING THE RISK OF HEREDITARY CANCERS: SOME PSYCHOSOCIAL ISSUES [226]}

\section{N. Hallowell}

University of Edinburgh, United Kingdom

Men and women who have a number of relatives with breast, ovarian and prostate cancer may be at risk of developing these diseases because they carry a dominantly inherited (BRCA1/2) mutation. These individuals need to reach a decision about managing their cancer risks. There are a number of risk management strategies available — which can be divided into familial and individual strategies. The former entails DNA screening - determining whether individuals and, subsequently, their relatives carry a mutation. The latter include bodily surveillance (breast, ovarian and prostate screening), chemoprevention (e.g., tamoxifen, oral contraception), risk-reducing presymptomatic surgery (e.g., mastectomy, oophorectomy), which are available to at-risk confirmed and unconfirmed mutation carriers. This presentation will present an overview of some of the main issues emerging from research that investigates the psychosocial implications of two risk management practices - genetic testing and risk-reducing surgery. I will argue that the process of managing risk has both an individual and familial dimension. Recommendations for future research will be made.

\section{THE NEW CYTOGENETICS — IMPLICATIONS FOR THE DIAGNOSTIC LABORATORY [110]}

S. Hamilton, S. Sane, N. Bain, and K. Fagan

Hunter Area Pathology Cytogenetics, John Hunter Hospital, Newcastle, Australia

Array CGH was introduced into our cytogenetics laboratory in 2007 on blood samples referred for development delay. So far we have reported 255 cases; our abnormality detection rate is $15 \%$ cf. $5.6 \%$ by conventional cytogenetics. During this time we have detected 'rare' abnormalities and seen first hand the emergence of 'new' syndromes such as deletion $16 \mathrm{p} 11.2$, deletion 1q41-42 and duplication MECP2 Syndrome. Platforms include both BAC and Oligo array systems, which has meant continuous development of hybridization and analysis techniques along the way. The problems encountered include the need to investigate abnormalities for significance by excluding known regions of polymorphism or CNVs. As this requires FISH validation and then follow-up of family members, the laboratory workflow needs to be altered to accommodate this. Skills in extracting and assessing DNA quality and labeling need to be acquired by all staff involved in producing array results so a good working relationship with your molecular genetics laboratory needs to be established. We have also introduced CGH analysis for tissue specimens from products of conception. The results show $100 \%$ success rate (vs. $85 \%$ for culture chromosomes) and an abnormality detection rate of $15 \%$. This has proven to be not only cost efficient but decreases turn around times for tissues. The combination of rapid aneuscreen FISH and arrayCGH means a result can be produced in less than 2 weeks. Furthermore, by eliminating the culture for chromosomes, fewer resources are required for tissue culture maintenance and these can be diverted into the microarray team.

\section{DEVELOPING A GENETICS MODULE FOR YEAR 10 HUMAN} BIOLOGY [179]

\section{J. Hancy, D. Dye, J. Brisbane, T. Charles, A. Samanek, and P. O'Leary}

Office of Population Health Genomics, Department of Health, Western Australia, Australia

Current research questions whether scientific knowledge can be attained by using DNA terminology and information-rich approaches. In addition, it is suggested that a balance between dispensing information and generating learning be employed when teaching science. The aim of this project has been to develop a human genetic information module in consultation with the Science Teachers Association of Western Australia Inc. The objective is to accurately describe difficult hereditary concepts in a way that promotes inquiry and understanding, and redresses misconceptions. For this reason, this module builds DNA concepts from the bottom-up, and contains suggested activities that students can relate to and explore 
using this information framework. To aid breaking down barriers surrounding difficult terminology and ambiguous synonyms a lexicon is contained in the front of most topics of this module. Associations linked to 'what students already know' are also included to lessen resistance to genetics. The Science Teachers Association of Western Australia Inc will market, distribute and advertise the Year 10 education module on human health genetics. Office of Population Health Genomics will retain editorial responsibility for the scientific accuracy of the genetics content.

\section{SHOULD WE BE SCREENING FOR KLINEFELTER SYNDROME? A CRITICAL ANALYSIS [133]}

\author{
A. S. Herlihy' ${ }^{1,2,3,4}$, J. Halliday ${ }^{1,5}$, M. Cock ${ }^{2,3}$, R. McLachlan ${ }^{2,3,4}$, and L. Gillam ${ }^{1,5}$ \\ Murdoch Children's Research Institute, Parkville, Australia \\ Monash University, Clayton, Australia \\ Andrology Australia, Clayton, Australia \\ Prince Henry's Institute of Medical Research, Clayton, Australia \\ University of Melbourne, Parkville, Australia
}

Recent rapid advances in genetic technology mean the range of conditions for which postnatal genetic screening could potentially be implemented have greatly increased. It is now possible to screen for conditions that could result in a spectrum of possible lifetime outcomes, which an individual may or may not experience. One such condition is Klinefelter Syndrome (KS), 47XXY. The prevalence of KS has been estimated at 1:650 males. Up to $70 \%$ of individuals remain undiagnosed, even though they may benefit from medical, educational and psychosocial interventions. Early detection has been advocated for many years but population-based genetic screening has never been explored. We explore here genetic screening for KS, and at what ages this might be appropriate. There are ethical, social, legal and economic issues associated with implementing such a program and it is vital these are clearly identified. We have developed an individual-specific framework for assessing the potential risks and benefits of screening, as a means of systematically identifying missing information. This framework is also relevant for assessing other variable genetic conditions. Our results show there is extensive literature regarding the medical aspects of $\mathrm{KS}$, but very little regarding the psychosocial impact of the condition. In addition, there is almost no information regarding the effectiveness of treatments and interventions and how they might relate to quality of life and life outcomes in adulthood. It is not possible to make a decision regarding screening for KS until this information is available. We have used these results to inform future research.

\section{'A WHOLE NEW WORLD': PARENTS EXPERIENCES AFTER CONTINUING A PREGNANCY WITH A GENETIC DIAGNOSIS [190]}

C. Hickerton 1.2, M.Aitken', J. Hodgson', and M. Delatycki',2

Murdoch Childrens Research Institute, Australia

Bruce Lefroy Centre for Genetic Health Research, Royal Children's Hospital, Parkville, Victoria, Australia

The ability to detect genetic conditions prenatally has expanded in recent decades. The majority of people choose to terminate an affected pregnancy after prenatal diagnosis but there are some people who choose to continue such a pregnancy or decline prenatal diagnosis where a high risk is known. Little is known about the experiences of families who choose to continue an affected pregnancy. This qualitative study involved in depth interviews with 9 parents who experienced a pregnancy where a genetic condition was, or could have been detected. Transcripts of the interviews were analysed using a grounded theory approach. Findings revealed 3 major themes: Finding out the diagnosis; living with the diagnosis; and attitudes to prenatal diagnosis and disability. The parents gave rich insights as they described the beginning of a new life path. It was found that while dealing with their own attitudes and getting on with their "new world', others' attitudes had an impact on their experience. A conceptual model arising from the major themes is offered as a new way of thinking about this paradigm. Parents who continue a pregnancy where a genetic condition is detected or suspected prenatally need appropriate support to assist with adjusting to their new life path.

\section{POPULATION BASED SCREENING FOR FRAGILE $X$} SYNDROME: OPTIONS, ISSUES AND CHALLENGES [107]

M. Hill', A.Archibald', B. Bennetts ${ }^{2}$, M. Field ${ }^{3}$, and S. Metcalfe ${ }^{1,4}$

Murdoch Childrens Research Institute, Melbourne, Australia

Department of Molecular Genetics, The Children's Hospital at Westmead, Sydney, Australia

GOLD Service (Genetics of Learning Disability) Hunter Genetics, Newcastle, Australia

Department of Paediatrics, University of Melbourne, Melbourne, Australia

In Australia a limited number of population-based genetic screening programs are carried out, most commonly during prenatal or newborn periods. With more than 1600 diagnostic genetic tests currently available, population-based screening will become increasingly prevalent into the future. It is therefore important to critically examine how screening programs can best be offered. Fragile X syndrome (FXS), an X-linked condition, is the leading cause of inherited intellectual disability. Genetic testing is available to identify individuals affected by FXS as well as carriers of the condition. The current approach to identifying affected individuals is through recognition of symptoms followed by genetic testing. Carriers are primarily identified through cascade testing of relatives of an individual diagnosed with FXS. Currently, both newborn screening and populationbased carrier screening (preconception and prenatal) are being studied, discussed and debated. Newborn screening aims to detect individuals affected by FXS to enable treatment initiation before symptom onset and will allow informed reproductive planning for future children. Carrier screening aims to provide carriers with reproductive options and information about their own health. Although these approaches have different purposes, they share ethical and social challenges in how best to deliver information, provide support and minimize harm. We review the existing literature and draw on our collective experiences of a carrier screening pilot study, a larger attitudinal study and a newborn screening pilot study to discuss the advantages and disadvantages of the different approaches. We give particular consideration to social, psychological and ethical aspects of implementing population-based screening programs for FXS.

\section{RIBOFLAVIN DEFICIENCY CAUSED BY DELETION OF THE RIBOFLAVIN TRANSPORTER, GPR172B, DETECTED BY QUANTITATIVE REAL-TIME PCR [215]}

G. Ho ${ }^{1,3}$, M.A. Chiong ${ }^{2,3}$, K. G. Sim ${ }^{2}$, K. Carpenter ${ }^{1,3}$,W. Rhead ${ }^{4}$, R. K. J. Olsen ${ }^{5}$, G. Peters ${ }^{2,3}$, and J. Christodoulou ${ }^{1,3}$

Genetic Metabolic Disorders Research Unit, Children's Hospital at Westmead, Sydney, Australia

Western Sydney Genetics Program, Children's Hospital at Westmead, Sydney, Australia Discipline of Paediatrics \& Child Health, University of Sydney, Sydney, Australia Medical Genetics Center, Children's Hospital of Wisconsin, Milwaukee, WI, United States of America

Research Unit for Molecular Medicine, Aarhus University Hospital and Faculty of Health Sciences, Skejby Sygehus, Aarhus, Denmark

Riboflavin, or vitamin B2, is a precursor to flavin adenine dinucleotide (FAD) and flavin mononucleotide (FMN), both used in biological oxidation-reduction systems. We previously reported a case of a newborn female who had clinical and biochemical features of multiple acyl-CoA dehydrogenation deficiency (MADD), which was corrected by riboflavin supplementation. Further investigations revealed that the mother was persistently riboflavin deficient, suggesting that a possible genetic defect in riboflavin transport in the mother was the cause of the transient MADD seen in the infant. G protein-coupled receptor 172B (GPR172B) has recently been shown to be a riboflavin transporter in humans and rats and is therefore considered a likely candidate gene for the riboflavin deficiency in the mother. The coding region of GPR172B in the mother was sequenced and two homozygous silent polymorphisms were identified. Using qRT-PCR, a deletion in GPR172B spanning multiple exons was identified in the riboflavin-deficient subject but not in her daughter. We postulate that this is the probable basis for the riboflavin deficiency. This is also the first instance of a defect found in the GPR172B gene.

\section{ENHANCING FAMILY COMMUNICATION ABOUT GENETICS:} DEVELOPMENT OF A COUNSELLING INTERVENTION [121]

\section{J. Hodgson and C. Gaff}

Murdoch Children's Institute, Royal Childrens Hospital, Melbourne, Victoria, Australia

Clinical genetics consultations often provide individuals with information that has implications for their family members. While health professionals frequently offer encouragement and support with such communication it is apparent that many relatives remain uninformed about relevant genetic information. However, specific counseling strategies to enhance family communication about genetics have not been systematically developed and evaluated. We present here the theoretical and iterative development of a genetic counseling strategy, designed to be delivered by telephone after a face-to-face consultation. It is informed by empirical data on barriers to family communication and aims to be clinically feasible, reproducible and grounded in appropriate counseling theory and genetic counseling tenets. The resulting intervention will be presented and critiqued. The ethical issues faced, and their resolution, will be discussed. The efficacy of the intervention is to be tested by means of a randomized controlled trial funded by NHMRC.

\section{EARLIER PRENATAL TESTING: HARMS AND BENEFITS [122]}

J. Hodgson ${ }^{1,2}$ and S. Metcalfe ${ }^{1,2}$

Department of Paediatrics, University of Melbourne, Australia

Murdoch Children's Research Institute, Parkville, Victoria, Australia

Noninvasive prenatal genetic testing for Down syndrome may benefit pregnant women by (a) enabling them to gain knowledge about the health 
of their fetus earlier in pregnancy than previously possible, (b) avoid the risk of miscarriage associated with invasive diagnostic tests such as chorionic villus sampling (CVS) and amniocentesis However provision of earlier testing raises a number of issues for both pregnant women and health professionals. Pregnancies identified with a chromosome anomaly are more likely to miscarry in the first trimester (12 weeks). This new form of testing will identify a number of affected fetuses at earlier stages of pregnancy and, as a result, greater numbers of women will be faced with the often emotional challenge of deciding whether to terminate a pregnancy that may have miscarried naturally. There is evidence that many women are currently not making informed choices about existing prenatal screening and diagnostic tests. It is vital for health professionals to consider how they will ensure that women are able to make informed choices about the earlier tests and the available options following diagnosis, including abortion. The authors suggest that in the absence of these necessary service provisions, earlier prenatal testing has the potential to create as many harms as benefits.

\section{MOLECULAR SCREENING APPROACH FOR IDENTIFICATION OF MUTATIONS CAUSING FAMILIAL HYPERCHOLESTEROLAEMIA IN WESTERN AUSTRALIA [196]}

A. J. Hooper', L.T. Nguyen', J. R. Burnett ${ }^{1,2}$, F. M. van Bockxmeer ${ }^{1,3}$ on behalf of the FHWA Pilot Program

Department of Core Clinical Pathology \& Biochemistry, Royal Perth Hospital, Australia ${ }^{2}$ School of Medicine \& Pharmacology, University of Western Australia, Australia ${ }^{3}$ School of Pathology \& Laboratory Medicine, University of Western Australia, Perth, Australia

Autosomal dominant familial hypercholesterolaemia $(\mathrm{FH})$ is associated with severe premature coronary heart disease (CHD) and affects some 10 million people worldwide. $\mathrm{FH}$ is caused by reduced plasma clearance of low-density lipoprotein (LDL) by the LDL-receptor pathway. FH is mainly caused by mutations in the $L D L R$ gene; over 800 have been described. Of the estimated 40,000 cases of FH in Australia, 90\% are undiagnosed and less than $10 \%$ are being adequately treated with statinbased therapy. The FH Western Australia (FHWA) pilot program has been established to identify and treat individuals with FH in WA, by family cascade screening from index cases. A genetic screening protocol has been designed that consists of three stages: (1) detection of 20 common mutations by an ARMS commercial kit (Tepnel), (2) detection of deletions/duplications by MLPA (MRC-Holland) and (3) exon-by-exon sequencing of the LDLR. Using this approach, a mutation was identified in 49 of 63 index patients with a diagnosis of definite FH based on Dutch Lipid Clinic Criteria enrolled in FHWA pilot program. Fourteen patients carried mutations that were detected by ARMS, and six carried mutations detected by MLPA, indicating that almost half of FH index patients carrying a mutation could be identified without the need for exon-by-exon sequencing. In summary, we present a cost-effective genetic screening strategy for FH and preliminary data for the FHWA pilot program. DNA test results will be useful for cascade screening from FH index cases, leading to early detection of other previously undiagnosed family members and enabling early intervention and prevention of premature $\mathrm{CHD}$ and death.

\section{PERSISTENT DISAGREEMENT ABOUT BIOBANKS AND THE IMPACT ON PUBLIC POLICY ARISING FROM PUBLIC AND CONSUMER GROUP ENGAGEMENT [182]}

\section{F. Hope, C. Molster, and P. O'Leary \\ Office of Population Health Genomics, Department of Health, Western Australia, Australia}

Using a novel, deliberative approach to public engagement in policy development, four-day forums were held in August and November 2008 with 34 members of the general public and genetic support groups to examine the issue 'How should biobanks operate in Western Australia?' Prior to the forum, participants received a booklet on perspectives about biobanks. At each forum presentations were given by a range of people such as a biobank custodian, ethicist, lawyer, biobank participant, medical researcher, Aboriginal health researcher and a disability advocate. These presentations offered arguments for and against the use of biobanks, in order to encourage debate and deliberation in small and large group discussions. The information outcomes of the forums were a set of recommendations to policy-makers, and the identification of core values, hopes and concerns, areas of consensus and areas of persistent disagreement. This paper examines the relevance of these outcomes to policy decisions and the extent to which they provide clarity to decision-makers on how the public might resolve conflicts between trade-offs that might be required in relation to the complex issue of biobanks. Particular emphasis is placed on the areas of persistent disagreement.
EVALUATION OF TELEGENETICS IN DIAGNOSIS OF DEVELOPMENTAL DISABILITY [6]

\section{B. Hopper, M. Buckman, and M. Edwards}

Hunter New England Area Health Service, Australia

Telegenetics is a new development in the service delivery of Genetic Services in Australia. This project was designed to establish if it was an acceptable alternative to face-to-face assessment in the genetic assessment of intellectual disability including morphological assessment of the patient. Ten children from 2 outreach clinics in rural NSW who were referred and assessed by their paediatrician were assessed by a single geneticist via telehealth and then seen again face-to-face as a 'gold standard'. Satisfaction surveys were then sent to both the parents and the referring paediatricians. After the face-to-face appointment, the clinical geneticist reviewed the recordings of both the transmitted footage and the high definition footage that was sent separately. There were very few morphological findings missed by the telegenetic assessments. Discrepancies like this could decrease in frequency as staff become more familiar with the methods. The parents of the patients reported no problem with the cameras and telehealth. They would have preferred face-to-face appointment but would be happy to have the telehealth appointment if it meant being seen earlier. This pilot study might have implications for future service delivery options.

\section{EXPLORING HEALTH PROFESSIONAL'S VIEWS ON PERINATAL HOSPICE CARE; A QUALITATIVE STUDY [29]}

A. Horwood', S. Buscombe ${ }^{2}$, and J. Hodgson ${ }^{1,3}$

Department of Paediatrics, University of Melbourne, Australia

${ }^{2}$ Genetic Health Services Victoria, Royal Children's Hospital, Australia

Murdoch Childrens Research Institute, Parkville, Victoria, Australia

Pregnant women who receive a diagnosis of a lethal fetal abnormality may choose to either terminate their pregnancy or continue in the knowledge that their baby is expected to die before or shortly after birth. There is currently no published Australian literature about what care is available to women who choose to continue a pregnancy diagnosed with a lethal fetal abnormality. In other countries, a structured model of care, perinatal hospice care, may be offered in these circumstances. Perinatal hospice care involves provision of medical care and psychological support by a multidisciplinary team allowing families to engage with the ongoing pregnancy and value whatever time they may have with their baby. This presentation will describe the justification for and qualitative methods used for a Masters research project that aims to explore the views and experiences of health professionals about (1) current practice of care for women who choose to continue a pregnancy diagnosed with a lethal fetal abnormality, and (2) the acceptability of perinatal hospice care in Victoria. Findings from this project will provide a preliminary understanding of the care currently available for these women in Victoria, as well as health professional's views on the appropriateness of perinatal hospice care. This will hopefully encourage and facilitate further research into perinatal hospice care in Victoria.

\section{GENETIC COUNSELING FOR SCHIZOPHRENIA: A REVIEW OF REFERRALS TO A PROVINCIAL MEDICAL GENETICS PROGRAM FROM 1968-2007 [229]}

M.-J. Hunter', C. Hippman'2, W. G. Honer², and J.Austin ${ }^{2}$

Department of Medical Genetics, University of British Columbia, Vancouver, Canada

2 Department of Psychiatry, University of British Columbia, Vancouver, Canada

Schizophrenia is a common disorder affecting $1 \%$ of the population. Recent studies have shown that there is a great deal of interest in genetic counseling amongst individuals with schizophrenia and their family members, but that few have received this service. It is important for the genetic counseling profession to know who is being served, and who is not, so that we can identify barriers to service and improve access. We conducted an exploratory, retrospective study to describe the population of individuals who were referred to the British Columbia Provincial Medical Genetics (BCPMG) program for genetic counseling for a primary indication of schizophrenia, between 1968 and 2007. A search of the BCPMG program database was performed to identify referrals for a primary indication of schizophrenia. Charts were reviewed and a database was constructed containing information describing the following characteristics: type of referring physician, demographics (age, sex, ethnicity, geographical location), psychiatric, medical, and family history for the individual referred, and (if applicable) that of their partner and their current pregnancy history. These characteristics and numbers of referrals for a primary indication of schizophrenia over the past 40 years were described using descriptive statistics. Between 1968 and 2007, the BCPMG program received 288 referrals for schizophrenia. Most referrals were made: (a) for individuals who had a first-degree family member with schizophrenia, rather than for individuals who were personally affected, (b) for preconception counseling, and (c) by family physicians $(69 \%)$, 
with only $2 \%$ by psychiatrists. Numbers of referrals for schizophrenia have been small, and in the last 12 years, decreasing while the total number of referrals to the department has been increasing. In BC, individuals affected with schizophrenia and their family members are rarely referred for psychiatric genetic counseling. There is a need to identify barriers to referral for psychiatric genetic counseling and for the development of strategies to improve access and outreach to this population.

\section{A LOCAL DATABASE FOR VARIANTS FOR BREAST CANCER, COLON CANCER AND HUNTINGTON DISEASE [108]}

\section{Hyland' and J.MacMillan ${ }^{2}$}

Molecular Genetics Laboratory, Haematology, Pathology, Queensland, Australia

Genetic Health Queensland, Australia

A local LOVD database is used to collect de-identified data from current breast cancer BRCA1 and BRCA2 gene analyses, colon cancer APC, MLH1, MSH2 and MSH6 and Huntington HTT gene analyses. These data include laboratory and clinical fields. Variants are described using current HGVS nomenclature. Techniques used for analyses are included. The laboratory data for BRCA1 and BRCA2 is from 2007 to date. All laboratory data for APC, MLH1, MSH2 and MSH6 data is included. To date no clinical data was included. We await recommendations from InSiGHT for clinical data. The laboratory data for Huntington is from 2006 to date. Clinical data is limited to the nature of the pathology request and is either diagnostic or presymptomatic. The local LOVD is used as a record to determine the specific type and frequency of any variant for each individual gene analyses. Data is stored on a PC that is not connected to any network. The data will be available to upload to an Australian specific gene database and then to international databases. Continual updating of the local LOVD database was achieved using a modification of existing laboratory worksheets to produce tab-delineated datasets. The data is curated in house. We aim to trial the GDRC VariVis software to facilitate the curation.

\section{KEEPING SECRETS: THE ETHICAL DILEMMA'S IN WORKING} WITH A FAMILY THAT IS NOT COMMUNICATING [14]

\section{A. Ingrey}

Hunter Genetics, Hunter New England Health Service, NSW, Australia

TL, a 23-year-old woman, was referred by her general practitioner regarding a family history of ornithine transcarbamylase (OTC) deficiency. TL was 9 weeks pregnant and was interested in discussing risk assessment and prenatal testing. The case was complicated by a break down in communication within the family. TL's nephew had recently died of this condition and TL was no longer speaking with her sister AL. Our service was treating $\mathrm{AL}$ and aware of the mutation that had been found in this family. AL was not willing to release this information to the extended family. This case highlights the need for guidelines to allow genetic services to release relevant information to family members when it is needed to provide appropriate clinical care. However, the use of such methods may have been detrimental to our ongoing relationship with AL in a volatile family dynamic.

\section{ENHANCING GENETIC KNOWLEDGE OF HEALTH CARE PROFESSIONALS BY INCLUDING PUBLIC HEALTH GENETICS INTO AN MPH PROGRAMME [205]}

\section{K. M. Ip, J. M. Johnston, and T. H. Lam}

Department of Community Medicine, University of Hong Kong, People's Republic of China

Enhancing genetic knowledge of health care professionals is important for the successful translation of genetic findings into improving public health practice. We report here an attempt to introduce Public Health Genetics as an elective module into the curriculum of the MPH program in the University of Hong Kong since September 2007. The curriculum centered on highlighting the genetic and environmental interaction in determining disease susceptibility and covered the following subject areas: principles of genetics, epidemiology, and public health genomics; genetics in disease and disease prevention; genetics services in Asia, including neonatal screening and free fetal DNA testing; evaluation of genetic tests; genomics in different specific diseases including inherited metabolic diseases, haemoglobinopathies, mental health, and osteoporosis; pharmacogenetics; ethical, legal, social and policy issues in genetics, genetics from an international perspective, and influence of Chinese philosophy on ethical issues in genetics. Teaching faculties included experts from different areas from UK (PHG Foundation, Cambridge) and local universities and Department of Health in Hong Kong. Students' evaluation is generally satisfactory. When using a five point scale to grade their improvement in understanding various areas of genetics after the course, $79 \%$ of students chose the top two categories regarding the associations between genetics, disease and environment, 93\% regarding its impact on public health, policy and clinical practice, $75 \%$ regarding its influence on future practice of clinical medicine and public health services, $78 \%$ regarding the role of public health in translating developments in genetics into practice, and $74 \%$ regarding its ethical, legal, social and policy implications.

\section{DISPARITIES BETWEEN NEONATAL SCREENING PROGRAMMES IN ASIA PACIFIC COUNTRIES [202]}

\section{K. M. IP', T. H. Lam', and R. Zimmern}

' Department of Community Medicine, University of Hong Kong, People's Republic of China PHG Foundation, Cambridge, United Kingdom

Despite technological advances in laboratory testing for neonatal screening over the past few decades, establishment of such programs for clinical service has been slow in many countries. We performed a literature review to examine the current condition of disparity between neonatal screening programs in various countries in the Asian Pacific Region. New Zealand, Australia, Japan and Singapore were among the first few Asian countries developed newborn screening programs since the mid-1960s. Currently nationwide screening is being offered to all newborns in Australia, Bangladesh, China, Japan, Korea, Malaysia, New Zealand, Palau, Philippines, Taiwan and Vietnam. Screening programs with varying degrees of partial population coverage are also available in India, Indonesia, Myanmar, Pakistan, Sri Lanka, and Vietnam. There appears to be no organized newborn screening program in Nepal, Cambodia, Laos and the other Pacific Island nations. Number of condition screened for varied from one to eight. Congenital hypothyroidism is the only condition being screened in all programs. Other conditions being commonly screened for included Phenylketonuria, Galactosemia, and Glucose-6phosphate dehydrogenase deficiency. Extended screening using MS/MS for amino acid, fatty acid or organic acid disorders are being piloted in different countries but only currently available to full population in Australia, New Zealand, Singapore, and Taiwan. Common hindrances mentioned for starting screening programs included a lack of awareness by health practitioners, ethno-cultural issues in the community regarding screening, and a lack of commitment by the government regarding program funding and logistics, especially for countries having a high percentage of delivery outside hospitals.

\section{AN INTEGRATED MODEL FOR INTERPRETING MISSENSE VARIANTS/MUTATIONS IN THE LONG OT SYNDROME GENES IN FAMILIES AT RISK [206]}

P.A. James', I. Macciocca', L. Gordon², A. Davies ${ }^{3}$, D. Phelan ${ }^{4}$, and D. DuSart ${ }^{4}$

${ }^{\prime}$ Genetic Health Services Victoria, Australia

${ }^{2}$ Bioinformatics Department, Murdoch Children's Research Institute, Australia

${ }^{3}$ Department of Cardiology, Royal Children's Hospital, Australia

${ }^{4}$ Molecular Genetics Laboratory, Victorian Clinical Genetics Service, Australia

Finding a mutation in an ion channel gene in Long QT syndrome allows the accurate identification of other family members at risk of sudden death. Interpretation of genetic investigations is complicated due to the majority of mutations in Long QT syndrome being missense mutations, frequently novel, and difficult to distinguish from rare polymorphisms. We present a new model that combines an analysis of 5 independent areas: the proband's clinical phenotype, familial segregation, evolutionary conservation, amino acid properties and data from control samples to generate an overall likelihood ratio that an observed variation is a pathogenic mutation. The ability of the model to improve the interpretation of DNA sequencing results was examined in 50 families with a clinical diagnosis of Long QT syndrome. Complete sequencing of six LQT genes (LQT1,2,3,5,6 and 7) was performed and the integrated model used to analyze any missense variants. Likelihood ratios strongly favoring pathogenicity were demonstrated for previously described mutations as well as novel mutations clinically suspected to be pathogenic. For recurrent mutations (e.g., KCNQ1 G314S) the LR exceeded 1000:1 in favor of causality while known polymorphisms analyzed by the model showed LRs strongly against causation. The described model provides an objective tool to analyze missense variants in long QT syndrome, allowing accurate interpretation of novel variants and facilitating further of predictive testing in at risk families.

\section{GENETIC COUNSELLING IN A FORM ACCEPTABLE TO AN} ABORIGINAL COMMUNITY [27]

\section{Jenkins}

Molecular Medicine and Genetics Laboratory Concord Hospital, Australia

A neurological disorder that has been long recognized as endemic among the Aboriginal population of Groot Eylandt and Arnhem Land was shown in this laboratory twelve years ago to be Machado-Joseph Disease. Since then members of the affected families have contacted this laboratory for advice about prevention and treatment of the disorder. It is clear that even through the nature of the disease is now known and that it is a dominantly inherited disorder, little change in the disease epidemiology has occurred. The aim of this paper is to find a way to deliver 'genetic counseling' in a 
form acceptable to an Aboriginal community. The problem is exacerbated by the difference in the marriage structure of Aboriginal communities where single communities are divided into two marriage moieties. Traditional Western style nuclear families are rare so that delivery of genetic counseling is made difficult because it cannot be delivered through a family structure. Further difficulties are translating Western technology in a simplified form into an Aboriginal language. The plan is to modify existing methods for MJD genetic counseling into an Aboriginal program, which can be delivered by female indigenous health workers in health clinics of Arnhem Land, or directly to involve communities. Different appropriate means of communication is to be developed, implemented and evaluated, commencing with using those already found to be appropriate to the Aboriginal health clinics, such as primarily pictorial poster presentations and diagrams and story telling. Results will be evaluation by Aboriginal health workers, health clinics and communities themselves.

\section{LIMITATIONS OF GENOTYPING USING SNP MICROARRAY IN THE PRENATAL INVESTIGATION OF MENKES}

\section{SYNDROME [225]}

\section{B. Kamien and $\mathrm{H}$. Slater}

Genetic Health, Queensland, Australia

Menkes disease is a disorder of copper transport caused by mutations in the copper-transporting ATPase gene (ATP7A- 23 exons spanning $150 \mathrm{~kb}$ genomic DNA). One third of affected males have Menkes disease as the result of a de novo mutation. MLPA analysis can detect large ATP7A deletions. We report a case of a woman with a previously affected male who underwent prenatal testing using the Affymetrix SNP 6.0 microarray as the treating team was unaware of the availability of more definitive genetic testing. The SNP analysis showed that 4 markers within the ATP7A gene were uninformative in this family due tohomozygosity. The first heterozygous markers that flanked the gene indicated the fetus had inherited the same genotype as the affected brother. For regions flanking both these markers, recombinants were identified at the next informative SNPs such that the fetus had inherited the opposite genotype from the affected brother. Caution was advised in interpretation due to the unlikely occurrence of two recombination events separated by a short genetic distance. Subsequent MLPA analysis of the affected child detected deletions of exons 2-17. These deletions were not present in the mother or fetus. This highlighted a limitation of SNP microarray as no markers should have been detected in the deleted areas of the gene.

\section{THE ABC OF PGD: A PROSPECTIVE STUDY EXPLORING THE 'LIVED EXPERIENCE' OF PREIMPLANTATION GENETIC} DIAGNOSIS [43]

\author{
J. Karatas ${ }^{1,2}$, K. Barlow-Stewart ${ }^{1,2}$, B. Mesier ${ }^{3}$, K. Strong ${ }^{4}$, C. McMahon ${ }^{5}$, \\ and C. Roberts ${ }^{6}$ \\ ${ }^{\prime}$ Centre for Genetics Education, Royal North Shore Hospital, Australic \\ ${ }^{2}$ Northern Clinical School, Faculty of Medicine, University of Sydney, Australia \\ ${ }^{3}$ Psychosocial research Group, Department of Medical Oncology, Australia \\ ${ }^{4}$ Centre for Values, Ethics and Law in Medicine, University of Sydney, Australia \\ ${ }^{5}$ Department of Psychology, Macquarie University, Australia \\ ${ }^{6}$ Sydney IVF, Sydney IVF, NSW, Australia
}

Preimplantation genetic diagnosis (PGD) that tests embryos for known genetic changes in the family prior to implantation using in-vitro fertilization (IVF) is a treatment pathway that is being used by some at-risk couples. This pathway is not always smooth however and may either result in establishing a pregnancy or leaving PGD treatment without success. While the psychosocial impact of IVF has been well studied, knowledge of how using PGD in addition to IVF impacts has to date been very limited. This paper will present data from a 3-year prospective follow up study of a sample of 45 women using PGD. Data from this study will be used to (1) illustrate the various and sometimes convoluted PGD pathways including the number of full stimulation cycles, expected and actual embryo transfers and outcome of pregnancy tests of 45 women; (2) describe the range of genetic conditions for which PGD was used; and (3) report on findings from the qualitative component which included 14 semi-structured interviews which have been audio-taped and transcribed. Thematic analysis has provided rich descriptions of how this group of women experienced PGD and the impact of their journeys including the effect on relationships; thoughts about embryos and the child born after PGD; societal stigma of IVF and PGD; expectations; and their information and support needs. The findings from this first ever study of the 'lived experience' of this reproductive technology will inform genetic counselling practice with clients for whom PGD may be an option.
NDUFS6-/- MICE AS A MODEL FOR MITOCHONDRIAL COMPLEX I DEFICIENCY [116]

B.-X. Ke', A. Laskowski', B. Hardman', D. Grubb', F. Rodda', M. McKenzie², M.T. Ryan', and D. R.Thorburn'

Mitochondrial and Metabolic Research Group, Murdoch Childrens Research Institute and VCGS Pathology, Royal Children's Hospital, and Department of Paediatrics, University of Melbourne, Parkville, Australia

${ }^{2}$ Biochemistry Department, La Trobe University, Bundoora Victoria, Australia

Mitochondrial Complex I deficiency is the most common mitochondrial enzyme defect in humans. Mutations in the NDUFS6 gene, encoding one of the 45 subunits of the $\sim 1,000 \mathrm{kDa}$ Complex I, are one cause of Complex I deficiency. NDUFS6-/- patients suffer from seizures, hypotonia and abnormal eye movements and die in the first month of life. Currently, no effective treatment for mitochondrial disease is available, emphasizing the need for accurate mouse models of Complex I deficiency. Here we describe a complex I deficient mouse model generated by knockout of the NDUFS6 gene. Ndufs6gt/gt mice were generated from GeneTrap embryonic stem cell lines and represent a partial knockout of the NDUFS6 subunit, with small amounts of wildtype mRNA detected in all tissues studied. For the first 6 months of life, Ndufs6gt/gt mice have no obvious phenotype, with normal growth rates and survival. Ndufs6gt/gt mice develop cardiomegaly, which can lead to sudden weight loss and lethargy after 6 months of age. Ndufs6gt/gt mice have marked Complex I deficiency in heart with more modest decreases in other tissues. Gross structure of brain is normal, however, the brainstem is thinner at the level of the Pontine Nucleus in Ndufs6gt/gt mice. Ndufs6gt/gt mice are a suitable model to better characterize the pathogenic mechanisms of complex I deficiency, particularly those related to cardiac problems. Treatment of mitochondrial disorders is currently inadequate and we anticipate that this mouse model will be useful for the study of new treatment strategies.

\section{HEREDITARY NONPOLYPOSIS COLORECTAL CANCER AND FAMILIAL ADENOMATOUS POLYPOSIS: INFORMATION DAY FOR FAMILIES [78]}

M. Kentwell', E. Lynch ${ }^{2,3}$, N. Gelfand ${ }^{3,4}$, M.A. Young 5 , F. Macrae', and K. Grant ${ }^{6}$

Familial Cancer Centre, Royal Melbourne Hospital, Australia

Familial Cancer Centre, Austin Hospital, Australia

${ }^{3}$ Genetic Health Services of Victoria, Royal Children's Hospital, Australia

${ }^{4}$ Familial Cancer Centre, Monash Medical Centre, Australia

Familial Cancer Centre, P. Mac, Australia

${ }^{6}$ The Cancer Council of Victoria, Australia

In 2007 and 2008, the Familial Cancer Centres across Victoria and the Cancer Council of Victoria jointly hosted information days for patients and their families with Hereditary Non-Polyposis Colorectal Cancer (HNPCC) and Familial Adenomatous Polyposis (FAP). An information day planning committee was formed and developed programs for the information days. The programs included genetic and medical information, current and future research information, psychosocial presentations, and discussion groups amongst the attendees facilitated by genetic counselors. The information days also included presentations by patients with HNPCC and FAP who shared their personal journey and experience before and following their diagnoses. A detailed evaluation process was conducted around the content, quality and delivery of each of the information days. This presentation will summarize the experience of developing and facilitating an information day for patients and families from Familial Cancer Centres across Victoria, as well as results from the evaluation questionnaire.

\section{NDUFS4-/- MICE AS A MODEL FOR MITOCHONDRIAL COMPLEX I DEFICIENCY [127]}

J. C. Komen', D.W. Leong', A. Laskowski', J. J. Pitt', M. McKenzie³, M.T. Ryan³,

H.S. Scott ${ }^{2,4}$, and D. R. Thorburn'

'Murdoch Childrens Research Institute and VCGS Pathology, Royal Children's Hospital, and Department of Paediatrics, University of Melbourne, Australia

Walter and Eliza Hall Institute of Medical Research, Melbourne, Australia Biochemistry Department, La Trobe University, Melbourne, Australia

${ }^{4}$ Institute of Medical and Veterinary Science and the Hanson Institute, and School of Medicine, University of Adelaide, Australia

Complex I of the mitochondrial respiratory chain is a $\sim 1,000 \mathrm{kDa}$ complex comprised of 45 subunits which are encoded by both the nuclear and mitochondrial DNA. A deficiency in Complex I activity is the most common mitochondrial enzyme defect in humans. Pathological mutations in the NDUFS4 gene, encoding one of the Complex I subunits, cause the neurodegenerative condition Leigh syndrome. Recently we have generated the first mouse model (Ndufs4-/- mice) for Complex I deficiency by a knockout of the NDUFS4 gene. Ndufs4-/- mice show temporary fur loss, growth retardation, unsteady gait and abnormal body posture when suspended by the tail. Biochemical analysis has shown that the mice have marked Complex I deficiency in all tissues tested. Blue Native-PAGE analysis of tissues from Ndufs4-/- mice shows a severe Complex I assem- 
bly defect, including the presence of a 'crippled' assembly intermediate also seen in tissues from children with NDUFS4 mutations. Metabolite analysis of the blood from Ndufs4-/- mice revealed an elevated alanine/ citrulline ratio and elevated levels of hydroxyacylcarnitines suggesting a decreased degradation of pyruvate and a decrease in mitochondrial NAD+ respectively. The phenotypical and biochemical data from this study indicate that Ndufs4-/- mice are a good model for Complex 1 deficiency in humans. We anticipate that this mouse model will be useful to better understand the pathogenic mechanisms of Complex 1 deficiency and mitochondrial disorders as a whole. Furthermore, as treatment of mitochondrial disorders is currently inadequate this mouse model provides an excellent starting point for the development of new therapies.

\section{ADDED CLINICAL VALUE OF BIOCHEMISTRY IN FIRST TRIMESTER SCREENING [214]}

P. Kumar, S. Khouri, T. Le, K. Chiang, and M. J. Sinosich

Prenatal Testing, Sonic Clinical Institute, Macquarie Park NSW, Australia

Prenatal screening in first trimester of pregnancy (10 to 13 completed weeks) is an accepted and established component of early assessment of feto-placental wellbeing. The screening program at Sonic Clinical Institute (SCI) incorporates four biochemical markers: pregnancy-associated plasma protein (PAPP) - A, free $\beta$ - subunit of human chorionic gonadotrophin (FßhCG), alphafetoprotein (AFP) and unconjugated estriol (uE3). In addition to screening for Down's syndrome affected pregnancies, the combination of fetal (AFP) and trophoblast derived markers (PAPP-A, $\mathrm{F} \beta \mathrm{hCG}$ ) frequently provides additional information about feto-placental physiology. All four markers were depressed in failed pregnancies with no fetal viability at ultrasound. However, in pregnancies which failed after normal ultrasound findings, both trophoblast-derived proteins (PAPP-A, $\mathrm{F} \beta \mathrm{hCG}$ ) were depressed and in pregnancies which progressed into second and third trimesters, despite a depressed PAPP-A, these were associated with fetal growth restriction. By contrast, in those pregnancies where subchorionic bleeding was confirmed at ultrasound, only AFP was significantly elevated (Median MoM $=4.02 ; n=15$ ). Finally, in pregnancies complicated with presence of trophoblastic disease, only F $\beta$ hCG levels were markedly elevated (Median MoM $=17.20 ; n=10$ ). These findings clearly demonstrate the added value of first trimester biochemistry, extending beyond screening for Down's syndrome.

\section{MINIMAL RESIDUAL DISEASE DETECTION AND GENOTYPING OF DRUG METABOLISING ENZYMES IN A COHORT OF CHILDREN WITH ACUTE LYMPHOBLASTIC LEUKAEMIA ENROLLED IN THE AUSTRALIA NEW ZEALAND CHILDREN'S CANCER STUDY GROUP STUDY VI CLINICAL TRIAL [213]}

E. Kwan, M. Norris, M. Haber, and G. Marshall

Sonic Clinical Institute, Macquarie Park, NSW, Australia

Acute lymphoblastic leukaemia (ALL) is the most common form of paediatric cancers. The outcome of children diagnosed with ALL has improved dramatically over the past 40 years resulting in remission rates of $97-99 \%$ and ultimate cure rates approaching $80 \%$, primarily due to modern multiagent chemotherapy. Despite this success, $20 \%$ to $30 \%$ of children diagnosed with ALL relapse to manifest signs and symptoms of the active disease. Such relapse is believed to be due to the persistence of small numbers of cancerous cells following chemotherapy and is often referred to as 'minimal residual disease' (MRD). Early, effective and sensitive detection of MRD may predict for impending relapse, which have major and immediate impact in the treatment outcomes of patients diagnosed with ALL. Relapse can also be attributed to the development of drug resistance in the leukaemic cell population. The mechanisms associated with chemotherapy response in paediatric ALL are not well understood such that resistant forms of ALL represent a leading cause of death. Yet there is accumulating evidence that a patient's genotype might influence the patient's response to multiple cytotoxic drugs and can be predictive of treatment outcome in childhood ALL. Polymorphisms in genes encoding for drug metabolising enzymes have also been shown to influence toxicity of drugs used in the treatment of cancers. This presentation is directed towards the study of MRD and single nucleotide polymorphisms (SNP) in children diagnosed with ALL who were enrolled in the Australia New Zealand Children's Cancer Study Group (ANZCCSG) Study VI clinical trial. It will also address the technological protocols used in MRD detection, especially in regards to the association of MRD detection and treatment outcomes.

\section{HLA B27 [210]}

\section{E. Kwan and M. Sinosich}

Sonic Clinical Institute, Macquarie Park, NSW, Australia

The Human Leucocyte Antigen B27 (HLA-B27) is strongly associated with spondyloarthropathies, a group of inflammatory rheumatic diseases, including ankylosing spondylitis (AS). Serological techniques such as enzyme immunoassays and flow cytometry are routinely used for typing HLA-B27. A prime disadvantage of these serological methods is the generation of false positive results, in particularly where the presence other HLA-B antigens such as HLA-B7 which can cross-react with HLAB-27. Hence, more accurate methods were developed for HLA-B27 typing, such as, real-time polymerase chain reaction (PCR), which allows for the simultaneous detection and quantitation of PCR products during thermal cycling. However, due to the inherent nature of the HLA-B27 subtypes where they differ only by a small number of nucleotide substitutions within exons 2 and 3 of the HLA-B27 gene, real-time PCR does not provide a $100 \%$ accuracy for detecting the presence or absence of the HLA-B27 genotype. A recent advancement in the genotyping of HLA is through the utilisation of sequence-specific oligonucleotide probes (SSO) bound to fluorescently labelled microspheres to identify alleles encoded by the sample DNA. We evaluated the LABType SSO Testing kit for the detection of the HLA-B27 genotype in 120 samples which were also by flow cytometry and a published real-time PCR method.

\section{COMMUNITY ENGAGEMENT FOR POLICY DEVELOPMENT: ARE HEALTH CONSUMERS REPRESENTATIVE OF THE GENERAL PUBLIC? [186]}

G. Kyne, C. Molster, and P. O'Leary

Office of Population Health Genomics, Department of Health, Western Australia, Australia

Community engagement aims to improve health policy and planning by identifying core citizen values and the opinions of a well-informed citizenry. Two deliberative forums were held over 4 weekends to determine 'How Biobanks should operate in WA'. The first event was held in August 2008 with 18 members of Genetic Support groups in WA. The second event was run in November 2008 with 16 randomly selected members of the Western Australian general public. The purpose of the forums was to identify core values, hopes and concerns relating to biobanks and to work toward consensus, identify areas of persistent disagreement and make recommendations to policy makers. The information outcomes of each forum were analysed using qualitative methodologies such as content analysis. While there were key areas of overlap, some aspects of the information outcomes from each event were quite different. Health Consumers were shown to place a stronger focus on community consultation and involvement in biobanks whereas the general public focused more on monitoring and review of the biobank. From the results it seems that each group had a different set of core values, hopes and concerns in relation to how biobanks should operate in WA. This suggests that Health Consumers are not representative of the wider community and identifies the importance of consulting with both during the process of policy development.

\section{YOUR BEST CHANCE OF A HEALTHY FUTURE MAY BE KNOWING ABOUT THE PAST: DEVELOPING EFFECTIVE TOOLS FOR THE PUBLIC TO COLLECT THEIR FAMILY HEALTH HISTORY [187]}

G. Kyne, C. Molster, S. Poke, J. Hancy, J. Brisbane, and P. O'Leary

Office of Population Health Genomics, Department of Health, Western Australia, Australia

Evidence supports the use of family health histories to determine risk stratification and prevention strategies for improving health outcomes, yet it appears to be underutilised by both members of the community and health professionals. The provision of tools to simplify the collection of family health history information, by both the community and by health professionals, are greatly needed. The design and structure of a family health history collection tool is recognised as a potential influence on the uptake and behavior change of the target group. The Office of Population Health Genomics has developed and evaluated a family health history collection tool for the general public. Best practice principles for the development of health promotion brochures and social marketing have been applied during the design process. The tool was developed and evaluated by applying the Trans-theoretical Model of Change, the PRECEDE-PROCEED framework and the Health Belief Model. Formative research methodology, focus groups and intercept surveys were used to identify factors that contribute to behavior change and encourage use of the tool. The results show the importance of implementation of best practice principles when designing a tool for the collection of family health history. Without consideration of the above mentioned principles the uptake and intended behavior change of the target group is greatly reduced. 
HUMAN SKELETAL MUSCLE ALPHA-ACTIN GENE (ACTA1) DISEASE UPDATE AND LOCUS SPECIFIC DATABASE [164]

N. G. Laing', D. E. Dye', G. Richard², N. Monnier', A. H. Beggs',

C.Wallgren-Pettersson ${ }^{5}$, and K.J. Nowak'

${ }^{\prime}$ Centre for Medical Research, University of Western Australia, Western Australian Institute for Medical Research, Australia

${ }^{2}$ GeneDx, 207 Perry Parkway Gaithersburg, United States of America

Laboratoire de Biochimie et Génétique Moléculaire, Inserm U607, Centre Hospitalier Universitaire de Grenoble, France

' Genomics Program and Division of Genetics and Department of Medicine, Children's Hospital Boston, Harvard Medical School, Boston, United States of America

${ }_{5}^{5}$ Carina Wallgren-Pettersson, Department of Medical Genetics, University of Helsinki and Folkhälsan, Finland

Diseases have been associated with 5 of the 6 human actin genes. The ACTA1 gene encodes skeletal muscle $\alpha$-actin, the principal actin isoform in the sarcomeric thin filaments of adult skeletal muscle. Interaction of this actin with myosins in the thick filaments produces muscle contraction and movement. Disease-causing mutations in ACTA1 were first described $(n=$ 15 ) in 1999. In this update we describe the currently known 174 different disease-causing mutations in ACTA1. ACTA1 mutations cause congenital myopathies: actin aggregate myopathy, intranuclear rod myopathy, nemaline myopathy, core myopathy and congenital fibre type disproportion. Biopsies from individual patients may show mixtures of the pathologies. Irrespective of the histopathology, the disease is usually clinically severe, with the majority of patients dying by one year of age. Most patients have de novo dominant mutations not present in the peripheral blood DNA of either parent. Mutations resulting in less severe disease, compatible with life into adulthood, may be dominantly inherited. The few, approximately $10 \%$, recessive mutations are genetic or, where tested, functional null mutations. In all recessive patients investigated there is retention in every skeletal muscle fibre of cardiac $\alpha$-actin, which is the predominant actin isoform in fetal skeletal muscle. Recessive ACTA1 disease severity inversely correlates with the level of expression of cardiac $\alpha$-actin. Although the pathogenicity of two variants is uncertain, there are as yet no amino-acid variants that are definitely non-disease causing polymorphisms. An ACTA1 Locus-Specific Database is to be curated at the Western Australian Institute for Medical Research (http://www.waimr.uwa.edu.au).

\section{FUS/CREB3L2 FUSION RING IN LOW GRADE FIBROMYXOID SARCOMA [163]}

\section{R. Lane, J. Peverall, A. Murch, and P. Robbins}

Cytogenetics Department, PathWest Laboratory's, King Edward Memorial Hospital, Perth, Western Australia, Australia

A sample from a buttock mass was cultured for cytogenetic analysis. Two cell lines were found, one with an apparently normal male karyotype and the other with a small supernumerary ring chromosome as sole abnormality. Histopathology suggested that this was a rare Low Grade Fibromyxoid Sarcoma (LGFMS), which can be hard to differentiate morphologically and immuno histochemically from other soft tissue tumours. Previous cytogenetic studies have shown that LGMFS is associated with a $\mathrm{t}(7: 16)(\mathrm{q} 33 ; \mathrm{p} 11)$ translocation with a smaller proportion of cases showing a supernumerary ring chromosome. FISH or RT-PCR have shown the $t(7 ; 16)$ to result in fusion between the FUS and CREB3L2 genes, and that in a very small number of cases FUS/CREB3L2 fusion could be shown in the supernumerary ring. In the present case FISH analysis showed two copies of the distal portion of the FUS gene and two copies of the Fusion between FUS and CREB3L2. The present case confirms that the presence of the fusion in a supernumerary chromosome is associated with Low Grade Fibromyxoid Sarcoma and is useful diagnostic tool for this rare tumour.

\section{ALPHA THALASSAEMIA TESTING: THE SOUTH AUSTRALIAN EXPERIENCE [139]}

C. Lau, A. Simsek, M. Holloway, Z. Kilpatrick, G. Casey, and Z. Rudzki

SA Pathology, Adelaide, South Australia, Australia

Alpha thalassaemia can be caused by deletions that remove one or both alpha globin genes. However, nondeletional forms also occur. The relative frequencies of the two types are not known in Australia. The aims are to determine the relative frequencies of the deletional and nondeletional forms of alpha thalassaemia in South Australia Method: Retrospective review of 776 patients referred for alpha thalassaemia carrier testing between Jan 2006 and Mar 2008. 53.1\% of the patients (412/776) carry common deletions. $39.9 \%(310 / 776)$ were carriers of single gene deletions $(217$ heterozygous, 93 homozygous), with -a3.7 being the most common type. $12.5 \%$ of the patients $(97 / 776)$ were carriers of double-gene deletions, with SEA being the most common. 34 deletion-positive patients also underwent alpha globin gene sequencing; causative mutations were identified in 6 $(17.6 \%)$. Of the remaining 364 deletion-negative patients, sequencing was undertaken in 265. Causative mutations were identified in $24.2 \%(64 / 265)$. 8 common mutations accounted for $70 \%$ (49/70) of the mutations detected. Identification of alpha globin sequence mutations led to the confirmation of carrier status in 70 patients ( $23.4 \%$ of all patients sequenced, $9.0 \%$ of total). This has important consequences for genetic/reproductive counseling. Therefore sequencing has an important role in alpha thalassaemia carrier testing. A small number of common mutations accounted for the majority of sequence variations. This opens the possibility of establishing a 'common mutation screen' to reduce the sequencing workload.

\section{AN INTERNATIONAL STANDARD CYTOGENETIC ARRAY (ISCA) CONSORTIUM AND DATABASE: POWER TO INVESTIGATE AUTISM AND OTHER DEVELOPMENTAL DISABILITIES [227]}

\section{H. Ledbetter}

Department of Human Genetics, Emory University, Atlanta, United States of America

High resolution, genome wide detection of gene dosage alterations (deletions, duplications), is rapidly being introduced into clinical cytogenetics on a variety of platforms including oligonucleotide aCGH, quantitative SNP microarrays and beadchips, collectively referred to as Cytogenetic Arrays. An international effort has been initiated to discuss standardization and guidelines for cytogenetic arrays, as well as a central, public database of cytogenetic array data from clinical laboratories. Two workshops held in June and December, 2008, and numerous conference phone calls, were used to compare and contrast 5 custom designs being used in $>$ 40 clinical cytogenetics laboratories in 5 countries. All laboratories involved are committed to open data sharing in a centralized, public database now being developed by the National Center for Biotechnology Information (NCBI) at NIH. The large volume of high quality cytogenetic array data obtained from patients with developmental delay, mental retardation and autism (currently in excess of 15,000 per year) will create a 'digital biobank', which will be an invaluable resource to both the clinical and research communities. For example, in our laboratory we have identified 11 deletions of $16 \mathrm{p} 11.2$, a region recently associated with autism. By surveying the consortium data, 11 additional cases were found, representing 1/300 clinical aCGH tests performed and identification of at least one new case of this microdeletion each week. Early assessments of these patients are revealing common features such as ASD, hypotonia, gastroesophageal reflux, anxiety and aggression. This work was funded in part by the 2008 ACMG Foundation-Luminex Award.

\section{IDENTIFICATION OF NOVEL HUMAN DISEASE GENES CAUSING COMPLEX IV (COX) DEFICIENCY [120]}

S. C. Lim', D. Kirby', R. Salemi', C. Zetzer', G. Smyth², M. Bahlo², and D. Thorburn'

'Murdoch Childrens Research Institute, Royal Children's Hospital \& Department of

Paediatrics, University of Melbourne, Australia

${ }^{2}$ The Walter and Eliza Hall Institute of Medical Research, Melbourne, Australia

Defects of the mitochondrial oxidative phosphorylation (OXPHOS) system occur in $\sim 1 / 5000$ live births and mostly affect organs with highenergy consumption. OXPHOS Complex IV (cytochrome c oxidase or COX) deficiency is one of the more common forms of OXPHOS deficiency. Mutations in genes encoding the mitochondrial DNA (mtDNA) or nuclear-encoded COX subunits are rare causes of COX deficiency. Most COX-deficient patients have mutations in nuclear genes encoding COX assembly factors such as SURF1. However, the molecular basis of approximately half of all COX deficient patients remains unknown. We aimed to identify the underlying genetic defect(s) of $8 \mathrm{COX}$ deficient patients from 6 unrelated Lebanese families, 4 of which were known to be consanguineous. These patients have a distinct from of Leigh Syndrome, the most common childhood presentation of mitochondrial disease. Complementation anlysis showed that all 8 patients have the same gene defect. Genome-wide SNP-typing using Affymetrix 50k Xba GeneChips was done on all affected individuals and some of their unaffected family members. Statistical linkage and haplotype analysis highlighted a $1.4 \mathrm{Mb}$ candidate region on chromosome 19. Genomic DNA and cDNA sequencing of 6 genes within and around the candidate region has not yet identified any pathogenic mutation. Microcell-mediated chromosome transfer (MMCT) was done to provide absolute biological evidence for the chromosome of interest. The COX enzyme defect of microcell hybrids should be corrected if chromosome 19 contains the gene of interest. Gene expression analysis was done on 2 affected siblings and their unaffected sibling using Affymetrix Human Gene 1.0 ST arrays.

\section{THE WA DNA BANK: AN NHMRC ENABLING RESOURCE FOR MEDICAL RESEARCHERS IN WESTERN AUSTRALIA AND BEYOND [194]}

\section{Macnish}

Centre for Genetic Epidemiology \& Biostatistics, University of Western Australia, Australia

The Western Australian DNA Bank (WADB) is a National Health and Medical Research Council (NHMRC) Enabling facility which provides the infrastructure, consumables and laboratory personnel for low cost biospecimen processing and dual-site storage of human DNA samples in Western 
Australia for medical research purposes. The WADB itself does not recruit these donors, but stores DNA for medical researcher's who have collected DNA samples from consenting donors participating in ethically approved research studies. A key aim of the WADB is to facilitate access to human DNA collections by the broader Western Australian and Australian research communities. By encouraging more medical researchers to use the WADB facilities it is anticipated that significant new initiatives and collaborations relevant to gene discovery, clinical and genetic epidemiology, new therapies and preventive medicine will ensue at the national and international level. The WADB has established a common storage and management system for biospecimens in WA that meets best-practice standards. An overview of how the WADB operates and how medical researchers in Western Australia, as well as the broader Australian research community, could make use of this facility will be presented.

\section{SKEWED MEIOTIC SEGREGATION IN A MALE WITH THREE} COPIES OF D15Z1 [26]

\section{N. Martin, J. Irving, and M. Flynn}

Queensland Fertility Group, Brisbane, Queensland, Australia

A 26-year-old male presented with a 22-month history of infertility after the birth of 2 children. Investigations included semen analysis and karyotype. Semen analysis on 2 occasions showed motility in the normal range, but $95 \%$ abnormal morphology and a count ranging from $12.7 \times 10^{6}$ to $17 \times 10^{6}$ per ml. AIH had failed in 2007 . His blood karyoytpe showed an extra small bisatellited marker chromosome. FISH demonstrated that it was chromosome 15 centromere negative and chromosome 14/22 centromere positive. However, three D group chromosomes (1 x 14 and $2 \times$ 15) were positive for the green Vysis centromere probe (D15Z1 at $15 \mathrm{p} 11.2$ ), but only two (both 15s) were positive for the Vysis red centromere probe (D15Z4 at 15p11.1-q11.1). Sperm FISH (874 sperm) analysis for chromosomes 14 and 15 was undertaken to establish whether IVF would be a viable option for this couple. Chromosomes were marked with probes so that 14 , der (14) and both 15 s could be identified on interphase. The der(14) (14 cen + D15Z1 positive) rarely segregated at meiosis, with $50 \%$ of sperm having nullisomy 14 . Those sperm that had a normal complement rarely contained the normal $15+$ der(14). The marker chromosome segregated in sperm, however, there were also abnormal segregation patterns for $14 / 22$ and the marker. The unusual meiotic segregation pattern would account for the patient's reduced fertility.

\section{ECONOMIC EVALUATION OF CYSTIC FIBROSIS PRENATAL} CARRIER SCREENING [176]

\section{S. Maxwell', L.Youngs', K. Brameld', E. Geelhoed ${ }^{2}$, and P. O'Leary}

Office of Population Health Genomics, Department of Health, Perth, Western Australia, Australia

School of Population Health, University of Western Australia, Australia

Cystic fibrosis (CF) is a serious, recessive, genetic condition affecting one in every 2500 individuals, with a carrier frequency of one in 25. Prenatal carrier screening, which aims to identify CF pregnancies is not routinely offered in Australia. Our study examined the cost effectiveness of two models of prenatal $\mathrm{CF}$ carrier screening compared to no screening over a one year period from a public health sector perspective. The two screening models were: (1) Two-step - the woman is screened, and if positive her partner is tested; or (2) Expanded one-step - the couple is offered testing simultaneously, results are reported to the couple and individually. Costeffectiveness was determined for a publicly funded carrier-screening program for both screening models and for a fee-for-service program for the 2-step model. A decision tree was generated using decision-modeling software (TreeAge Pro 2008) to estimate costs and outcomes for each scenario. Sensitivity analysis was undertaken to assess the impact of variation in model probabilities, such as participation rates, reproductive choices, and cost estimates. Under baseline assumptions a 1-year screening program (38 000 clinically recognized pregnancies) will identify 1,6 , and $7 \mathrm{CF}$ pregnancies for two-step fee-for-service, two-step public funding and expanded one-step public funding models respectively. The results of the analysis will help to inform the development of policy.

\section{COMMUNITY VIEWS ON THE COMMUNICATION OF FH GENETIC INFORMATION WITHIN FAMILIES [177]}

\section{S. Maxwell, C. Molster, S. Poke, and P. O'Leary}

Office of Population Health Genomics, Department of Health, on behalf of the Familial Hypercholesterolaemia (FH) Pilot Cascade Screening Program

The process of communicating genetic information to relatives where index cases have given consent has received little attention in policies and guidelines. Our study investigated the community's views on contact and communication within cascade screening practices, in situations where index cases have agreed to their relatives being informed. We explored community views on cascade screening for Familial Hypercholesterolaemia (FH), focusing on the desire of relatives to be informed about a familial risk, the acceptability of being informed by family and by health professionals and preferences around how this information is shared. We conducted a cross sectional, Computer Assisted Telephone Interviewing (CATI) survey. 430 respondents were recruited randomly from the electronic version of the Western Australian telephone directory. Most respondents indicated a desire to be informed about their familial risk of $\mathrm{FH}$ (93\%), to be informed by their relative $(91 \%)$ or the health clinic involved in the FH screening program $(77 \%)$ and be offered screening for the condition $(95 \%)$. Women aged $18-54$ years were more likely to report a preference for being informed by their relative. This study provides evidence of community support for $\mathrm{FH}$ cascade screening programs to adopt a policy of 'direct contact', where program staff contact the relatives of index cases and inform them of their familial risk. Maximizing community support for the screening program may require asking index cases for consent to include their personal details when the initial contact is made with their relatives.

\section{A MULTI-GENERATIONAL EXPLORATION OF CARRIER TESTING AMONGST FAMILY MEMBERS OF CHILDREN WITH CYSTIC FIBROSIS [112]}

B. J. McClaren ${ }^{1,2,4}$, M.Aitken', D. J.Amor ${ }^{1,2,4}$, R. J. Massie ${ }^{1,2,3,4}$, and S.A. Metcalfe $\mathrm{e}^{1,4}$

${ }^{1}$ Murdoch Childrens Research Institute, Australia

${ }^{2}$ Genetic Health Services Victoria, Australia

${ }^{3}$ Royal Children's Hospital, Melbourne, Australia

${ }^{4}$ The University of Melbourne, Australia

In Victoria, 20 babies are born with cystic fibrosis (CF) each year, making it the most common, severe, autosomal recessive condition in children. Family members of these children may wish to learn their carrier status, and can be tested free of charge. Uptake of testing is relatively low given the individual risk estimates. There has been limited exploration of the underlying influences of uptake of carrier testing; this study presents the first multi-generational survey of family members of children with $\mathrm{CF}$. The questionnaire developed for this study assesses knowledge of CF and carrier testing, attitude towards carrier testing, factors that influence a decision about carrier testing, and demographic information (age, sex, education, parity, future reproductive plans). The questionnaire has been sent to 178 family members, with $116(65 \%)$ returned to date; recruitment for this study is ongoing. Respondent demographics are: male;female $(36 \% ; 64 \%)$, tested;not tested $(44 \% ; 56 \%)$, grandparents;aunt or uncle;parent;second cousin;great aunt or uncle;first cousin;sibling $(29 \% ; 27 \% ; 19 \% ; 13 \% ; 6 \% ; 4 \% ; 2 \%)$. Preliminary analysis suggests positive attitude is not a predictor of carrier testing. The majority of respondents answered greater than 9 knowledge questions correctly (max. score 12 correct). The most commonly cited factor that may influence a decision to have carrier testing was 'if my partner was a carrier', and a decision to not have carrier testing was 'because I do not plan to have any (more) children'. Exploring the influences and predictors of uptake will inform clinical service delivery around offering carrier testing to family members.

\section{'I'VE DONE HEAPS OF RESEARCH ON THE INTERNET': INFORMATION SOURCES AND INTERNET USE AMONG NZ WOMEN AT HIGH RISK OF HEREDITARY OVARIAN CANCER [25]}

\section{A. McEwen}

Central \& Southern Regional Genetic Services, Wellington Hospital, New Zealand

Background: $80 \%$ of Australasians have Internet access. US data indicates $63.7 \%$ of internet users use the internet to seek health-related information. Health-related Internet searches are more common among people under 65, women, and those with higher levels of education and income. Cancer patients use the Internet to access information and seek online support. The aim is to report on information sources and Internet use among an NZ cohort of women with an increased risk of ovarian cancer. As part of a qualitative interview study of $32 \mathrm{NZ}$ women living with an increased risk of ovarian cancer we asked about sources of information and whether they trusted those sources. Information sources include doctors, genetic counselors, Internet, books, pamphlets, newspapers, Cancer Society, friends, family, at risk women and television. 18/32 (56\%) used the internet as a source of information. 4/32 reported choosing not to use the internet because they did not want to scare themselves or feel overwhelmed by information. 3/32 used the Internet to check the validity of information given to them by doctors. A few respondents said they did not trust all Internet information and it was important to choose the sites you visited. Some NZ women at high risk of ovarian cancer use the Internet to seek information and support about inherited cancer risk. A few women reported understanding Internet information could be inaccurate and indicated they used the Internet with caution. Health professionals need to find ways of facilitating Internet use to ensure patients locate quality information. 


\section{CONFINED PLACENTAL MOSAICISM ASSOCIATED WITH} LOW PAPP-A [165]

\section{A. Mclnerney-Leo and B. Sutton}

Specialised obstetrics + gynaecological imaging (so+gi), Brisbane, Queensland, Australia

To report 4 cases of confined placental mosaicism (CPM) associated with a low PAPP-A. Retrospective review of CVS results over the past 3 years. When CPM was identified first trimester biochemistry was also evaluated along with outcome data, where available. Results: 92 CVS procedures were performed over a 3-year period and four cases of CPM were identified. The karyotypes were as follows; $47, \mathrm{XX}+16$ (majority); 47, $\mathrm{XY}+2$ (48)/46, XY (18); 92 XXXX (10)/46, XX(87); 45, X (64)/46, XX (36). The PAPP-A levels were 0.06 MOM, 0.73 MOM, 0.26 MOM and 0.07 MOM respectively. The average was 0.28 MOM. Free Beta-hCG levels ranged from $0.15 \mathrm{MOM}$ to $1.73 \mathrm{MOM}$ with an average of $1.07 \mathrm{MOM}$. Three couples had a follow-up amniocentesis that was normal. Outcome is known in 3 cases and 1 pregnancy is ongoing (45,X mosaic). The pregnancy with mosaic trisomy 16 was induced at 33 weeks for IUGR and this female continues to grow below the 2 nd centile at 14 months but she has achieved normal motor and cognitive milestones. Both the trisomy 2 and tetrasomy mosaic cases went to term and produced babies with an average birthweight. They are 5 months and 10 months of age respectively with no developmental issues thus far. Conclusion: We have observed an interesting pattern of a low PAPP-A in 4 cases of CPM. Further large-scale studies will be initiated to see whether these levels differ significantly from PAPP-A levels in pregnancies with a normal CVS karyotype.

\section{EXON SKIPPING STRATEGIES TO ADDRESS DMD-CAUSING MUTATIONS: PERSONALIZED GENETIC THERAPIES [144]}

P. Meloni, R. Johnsen, K. Greer, S. Forrest, L. Stone, S. Fletcher, and S.Wilton Molecular Genetic Therapy Group, CNND, University of Western Australia, Perth, Australia

Duchenne muscular dystrophy (DMD) is a severe and progressive muscle wasting disease, caused by protein truncating mutations, and ultimately the loss of functional dystrophin in muscle. Becker MD (BMD), an allelic condition is generally caused by in-frame deletions within the dystrophin gene, resulting in the production of an internally truncated but variably functional protein. Antisense-oligonucleotide (AO) induced-exonskipping has the potential to by-pass protein truncating mutations and restore dystrophin expression. To date, the focus has been on addressing genomic deletions occurring in the major deletion hotspot. Our laboratory has designed and optimized a series of AOs to excise individual dystrophin exons 2 to 78 during processing of the dystrophin pre-mRNA, which have been evaluated in normal cells. We describe exon skipping strategies to rescue dystrophin expression in patient cell lines with different mutations (exonic duplications, micro-insertion/deletions, nonsense and splice motif defects), which can only be assessed in the appropriate patient cell line. In some cases, multiple strategies may be applicable to address one mutation, and it will be crucial to identify which approach will result in the most functional dystrophin isoform.

\section{THE ABORTION DEBATE: ENSURING WOMEN HAVE ACCESS [106]}

M.A. Menezes ${ }^{1,2}$, L. Skene ${ }^{3}$, J. M. Hodgson', M. Sahhar ${ }^{1,2,4}$, and S. A. Metcalfe ${ }^{1,2}$

Murdoch Childrens Research Institute, Australia

2 Department of Paediatrics, The University of Melbourne, Australia

${ }^{3}$ Melbourne Law School, The University of Melbourne, Australia

${ }^{4}$ Genetic Health Services Victoria, Australia

Abortion law reform has been foremost in the public eye in recent months with the Victorian parliament passing the Abortion Law Reform Bill 2008 (Vic) after much heated debate. In particular there was a great deal of controversy over the 'conscience clause' that requires doctors who are 'conscientious objectors' to refer women seeking an abortion to another practitioner. An estimated 100,000 abortions take place in Australia every year with $2 \%$ for fetal anomaly. Healthcare professionals working in prenatal settings are often involved in counseling clients during crisis situations following the diagnosis of a fetal anomaly. This potentially raises ethical, moral and legal issues for healthcare professionals working in this field. In-depth interviews are being conducted with healthcare professionals who work with couples undergoing a diagnosis of a fetal anomaly. To date, 18 healthcare professionals have participated in the study from Australia and Canada, and this study is ongoing. Participants identified a range of challenging situations and several discussed barriers to the access of abortion services. While the law may permit abortion there are non-legal obstacles that exist for women accessing abortion in both Australia and Canada that may impact on both clients and healthcare professionals. We would not suggest that doctors should be forced to perform procedures they feel are unethical. However, healthcare professionals and organizations need to be much more explicit about their position regarding provision of abortion services to ensure that women are supported and have appropriate access.
THE CHALLENGES OF SUPPORTING CLIENTS IN CRISIS: EXPERIENCES OF PRENATAL GENETIC COUNSELLORS [138]

\section{Menezes ${ }^{1,2}$, M. Sahhar ${ }^{1,2,3}$, J. Hodgson ${ }^{1,2}$, and S.A. Metcalfe ${ }^{1,2}$}

'Murdoch Childrens Research Institute, Australia

Department of Paediatrics, The University of Melbourne, Australia

${ }_{3}^{3}$ Genetic Health Services Victoria, Australia

The area of prenatal genetics has seen major technological developments in the past few years that have enabled more accurate screening and diagnostic tests aimed at detecting fetal anomaly during pregnancy. This has consequently led to increases in the number of fetal anomalies that are identified and diagnosed prenatally. Genetic counselors working in prenatal settings are often involved in counseling clients during crisis situations following the diagnosis of a fetal anomaly. This potentially raises ethical, moral and legal issues for healthcare professionals working in this field. It has been acknowledged that repeated empathic engagement with distressed clients can lead to feelings of stress and/or 'burnout'. In-depth interviews are being conducted with healthcare professionals who work with couples undergoing a diagnosis of fetal anomaly. To date, 15 genetic counselors have participated in the study from Australia and Canada, and this study is ongoing. Participants identified a range of challenging situations that have an impact on their daily lives, including ethical and moral dilemmas they encounter due to the nature of their work. Several participants discussed barriers to women's access of abortion services, which can impact on clients and healthcare professionals. Australian genetic counselors discussed formal and informal supports they use to deal with the challenges of their work, and the advantages and disadvantages of supervision. In contrast, Canadian genetic counselors only discussed their use of informal supports and saw supervision as a process mainly required by students and new counselors.

\section{USEFULNESS OF UPDATING DISEASE RISKS IN COMMERCIAL GENOME-WIDE SCANS FOR COMMON DISEASES [62]}

R. Mihaescu', M. van Hoek², E. J. G. Sijbrands², A. G. Uitterlinden²,

J. C. M.Witteman', A. Hofman', C. M. van Duijn', and A. C. J.W. Janssens'

Erasmus University Medical Center Rotterdam, Australia

Department of Epidemiology, Department of Internal Medicine, The Netherlands

Commercial companies selling online genome-wide scans for prediction of risk for common diseases update risk estimates on every new gene discovery. To assess the clinical utility of updating risk information, we compared type 2 diabetes (T2D) risk prediction based on one gene (TCF7L2), 18 genes, and 18 genes plus age, sex and body mass index in a prospective, population-based study among individuals of 55 years and older. Primary outcome was the percentage of individuals that changed from increased to decreased risk or vice versa. Type 2 diabetes risk in the Rotterdam study was $20 \%$. Predicted risk were slightly decreased for carriers of TCF 72 CC genotype (predicted risk 17.6\%) and slightly increased for the CT and TT genotypes $(20.8 \%$ and $28.0 \%)$. Based on testing 18 polymorphisms $24 \%$ of the CC carriers changed to increased risk and $6 \%$ of the TT carriers changed to decreased risk. These percentages were $29 \%$ and $19 \%$ when prediction was additionally based on age, sex and body mass index. In total, $50 \%$ of all participants changed from being at increased risk to decreased risk or vice versa. These changes could easily occur because most participants had disease risks that were close to the average risk. Updating risk predictions may provide contradictory information about the individual's risk status over time, which is undesirable if lifestyle and nutritional recommendations vary accordingly. Risk prediction from genome-wide scans is not useful for preventive applications at this stage.

\section{MUTATION LOAD OF BOTH FLT3-ITD AND NPM1 CORRELATES WITH CLINICAL OUTCOMES IN NORMAL KARYOTYPE AML [13]}

\section{K. Mina', M.Wright' ${ }^{2}$, M. Higgins' ${ }^{2}$, J. O'Reilly', and P. Cannell ${ }^{2}$}

PathWest, Australia

2 Department of Haematology, Royal Perth Hospital, Australia

Karyotypic abnormalities confer major prognostic significance in AML. Cytogenetically normal AML (CN-AML) poses intermediate risk but represents a diverse patient group. Research demonstrates that the presence of FLT3-ITDs and NPM1 mutations confer inferior and superior survival in CN-AML respectively, and mutant load and size may also have prognostic significance. Thus application of these molecular markers may further stratify $\mathrm{CN}-\mathrm{AML}$ and facilitate clinical decision-making. The aim of this study is to investigate the prognostic utility of FLT3ITD and NPM1 mutations in terms of positivity, mutation load and size. We tested 59 archived CN-AML patient samples, for which clinical data was available, for FLT3-ITD and NPM1 mutations and related these results to achievement of complete remission (CR), disease-free (DFS) and overall survival (OS). Mutation detection was carried out using PCR and fragment analy- 
sis by Genescan. NPM1 positivity and mutant:wildtype load threshold of $>50 \%$ were associated with improved odds of achieving CR, and NPM1 mutant:wildtype load threshold of $>60 \%$ was associated with improved OS. In those who achieved CR, FLT3-ITD mutant:wildtype load $>80 \%$ was associated with poorer disease-free survival. This study supports the use of FLT3-ITD and NPM1 mutant load thresholds as indicators of prognosis in CN-AML and has significant implications for diagnostic reporting and clinical decision-making.

\section{HEALTH EFFECTS OF CONSANGUINITY IN BANGLADESH: ACCOUNTING FOR NON-RANDOM PARTNER SELECTION IN FIRST COUSIN MARRIAGES [59]}

\author{
A. M. Mobarak', M. lyigun' ${ }^{2}$ M. Nizam Khan ${ }^{2}$, and A. H. Bittles ${ }^{3}$ \\ Yale University, New Haven, United States of America \\ University of Colorado, Boulder, United States of America \\ Murdoch University and Edith Cowan University, Perth, Australia
}

Health outcomes of consanguinity usually are assessed by straightforward comparisons of morbidity and mortality in the offspring of consanguineous and non-consanguineous marriages. This approach does not allow for the possibility that families opting for a consanguineous marriage may have socio-economic characteristics that independently predispose their progeny to a particular morbidity profile. This hypothesis was examined in a retrospective survey of 5208 pregnancies reported by 306 first cousin and 305 non-consanguineous rural households in the Teknaf district of Bangladesh. Detailed information was obtained on socio-economic status and specific health outcomes during pregnancy, infancy, and from 1 to 15 years. Initial comparative analyses suggested that the offspring of first cousin unions were $2.7 \%$ less likely to survive, and $4.4 \%$ more likely to report health problems. But approximately $50 \%$ of the mortality gap was explained by differential immunization uptake, with consanguineous children much less likely to have been immunized. Since marriage with a biological relative can only occur if a partner of the right gender and age is available, quasi-random variations in the incidence of first cousin marriages were generated to isolate the causal effect of consanguinity. By accounting for unobservable differences between the two groups, the survival penalty of first cousin progeny increased to $3.6 \%$. The main implications are that the causal effects of consanguinity on health in Teknaf may be more detrimental than initial correlations would suggest, and the conclusions that can be drawn about consanguinity and health are statistically less precise than those implied by these simple correlations.

\section{EVALUATING DELIBERATIVE PUBLIC ENGAGEMENT FROM MULTIPLE PERSPECTIVES: IS IT AN EFFECTIVE METHODOLOGY FOR GENOMIC PUBLIC POLICY DEVELOPMENT? [199]}

\section{Molster', B. McNamara², T. Phillips², A. Potts², and P. O'Leary'}

Office of Population Health Genomics, Division of Public Health, Department of Health, Perth, Western Australia, Australia

Anthropology and Sociology, School of Social and Cultural Studies, University of Western Australia, Australia

Public health systems worldwide have expressed strategic commitments to foster and support consumer and community engagement in health system performance. For areas such as population health genomics, where the issues are often complex, ethically contentious and involve difficult choices or trade-offs among value-based options, innovative engagement methodologies have been proposed to obtain the underlying values and deliberated views of an informed citenzry. While numerous rationales have been put forward to support the use of these methodologies, there is little empirical evidence of their effectiveness in contributing to the legitimate and substantive quality of public policy. There is a paucity of evaluative frameworks and few deliberative public engagements have been comprehensively evaluated from multiple perspectives. This presented an opportunity to develop and apply a framework for the multi-dimensional evaluation of recent deliberative engagement forums on biobanking in Western Australia. Perspectives represented in the framework include forum participants, policy decision-makers and the broader general community. Findings from this study will inform the future development and application of public engagement methodologies within population health genomics.

\section{A BIOINFORMATICS APPROACH TO THE STUDY OF AUTOSOMAL RECESSIVE NON-SYNDROMIC INTELLECTUAL DISABILITY [55]}

P. Moolhuijzen, M. L. Black, M. Bellgard and A. H. Bittles

Centre for Comparative Genomics, Murdoch University, Perth, Australia

Although non-syndromic intellectual disability (NSID) affects large numbers of people worldwide, few data have been available on specific genetic aetiologies. This lack of information has hindered both carrier detection and premarital and prenatal counselling. The integration of diverse but inter-related data types is a current major challenge in bioinformatics. Trends and commonalities are difficult to identify and while manual summations are possible they inevitably require exhaustive effort. Our aim was to integrate data generated through a web-based system that allowed research results to be shared, interpreted and cross-linked. To test this approach, publications and reports abstracted from the PubMed and OMIM databases were systematically reviewed, potential autosomal recessive NSID loci identified, and the results mapped using CMap Comparative Map software from GMOD (Generic Model Organism Database project - http://www.gmod.org). CMap enables disparate data to be presented in a single integrated viewer, facilitating comparative analysis and providing a critical overview of NSID-related loci and genes for decision-making. To date 12 chromosomal regions (MRT1-12) associated with NSID have been identified, mostly through homozygosity mapping in large consanguineous kindreds. These regions vary in size from $25 \mathrm{~Kb}$ to $26 \mathrm{Mb}$ and are located across 10 autosomes. Genes positively associated with NSID genes include PRSS12 (MRT1, 4q26), GRIK2 (MTR6, 6q16.3q21), and CC2D1A (MTR3, 19p13.12), all of which encode receptor proteins involved in neurone biochemistry. In addition, overlaps between MRTs and reported associations with schizophrenia and autism strongly suggest that these regions of the genome merit detailed examination.

\section{PRENATAL DETECTION OF AN UNUSUAL RECOMBINANT CHROMOSOME (3) RESULTING FROM A MATERNAL COMPLEX CHROMOSOME REARRANGEMENT [130]}

\section{B. Morar}

Western Australian Institute for Medical Research, Nedlands, Western Australia, Australia

The study of Mendelian genetics in the Bulgarian Gypsies is highlighted by many successes over the past decade. More than 20 genetic disorders are caused by single founder mutation each in culturally and geographically diverse Gypsy sub-isolates. This is largely due to the nature of the Gypsy population: a recently formed isolate from a small number of founders. More recent studies of epilepsy have however produced results contrary to expectations: surprising levels of phenotypic and genetic heterogeneity in families from the same Gypsy group and also within the same family. Unrelated Gypsy controls and affected families have been genotyped with the Affymetrix SNP5.0 Array to identify causative epilepsy genes. An in-house $0.1 \mathrm{cM}$ linkage marker set polymorphic in the 68 controls has identified multiple positive signals in the genome, with no apparent positive regions shared between families. A three-generation family with GEFS+ (generalized epilepsy with febrile convulsions + ) belongs to a small sub-isolate, practicing consanguinity. We have obtained a positive LOD score $>3.5$ for a $6.63 \mathrm{cM}$ region on chromosome 12 using a dominant model with reduced penetrance. A recombination identified in an affected individual reduces the size of the region to $\sim 3.57 \mathrm{cM}$. No significant linkage disequilibrium is present between markers in the region and the linkage result withstands perturbation to model parameters. Additional samples from affected and unaffected family members are under investigation. A number of interesting genes lie within this region and their possible role in epilepsy is being analyzed.

\section{CONSANGUINEOUS COUPLES [39]}

\section{H. Mountain ${ }^{1,2}$ and I. Barns ${ }^{2}$}

Genetic Services of Western Australia, King Edward Memorial Hospital, Subiaco, Australia Institute of Science and Technology Policy, Murdoch University, Perth, Australia

Much of the literature on genetic counseling for consanguineous couples comes from countries and communities where cousin marriage is a culturally influenced and accepted practice. In a survey of 42 consanguineous couples presenting for genetic counseling in Western Australia and 27 consanguineous couples who had not received genetic counseling, we found the majority were from Western countries where close kin marriage is not common. There is limited information on community attitudes to consanguinity and how consanguineous couples in Australia have experienced or might benefit from genetic counseling. We conducted questionnaires exploring a range of issues including couples' experiences with being in a consanguineous relationship, risk perception, and their experiences with genetic counseling. Interviews with 16 couples were conducted to explore the issues in greater depth. Qualitative and quantitative data will be presented. The results provide valuable information about consanguineous couples lived experiences and how family and community attitudes have affected them. In many cases these issues outweighed any increased genetic risks. Genetic counselors are in a unique position to provide accurate risk estimation and factual information to empower couples to deal with society's adverse reactions to their relationship. 


\section{AN EXPLORATORY STUDY OF GENETIC COUNSELLING SESSIONS AND WHETHER CULTURAL DIFFERENCE AFFECT RAPPORT OR COMMUNICATION [40]}

\section{H. Mountain' and I. Barns ${ }^{2}$}

${ }^{\prime}$ Genetic services of Western Australia, King Edward Memorial Hospital, Subiaco, Australia ${ }^{2}$ Institute of Science and Technology Policy, Murdoch University, Western Australia, Australia There is limited information on what happens during genetic counseling sessions and how genetic counselors communicate with their clients. Genetic counseling was long thought of as a communication process where counselors imparted their knowledge of genetics, now we realize that the interaction between participants is much more complex. In the last 5 years there have been numerous studies describing the genetic counseling process with many concluding that counseling sessions are mostly didactic in nature with little time available to explore psychosocial and emotional topics. This exploratory study looked at the content of genetic counseling sessions and the factors that influence how genetic counselors and their clients interact and whether any perceived or actual cultural differences affected rapport between counselor and client. Genetic counseling sessions were recorded, transcribed and analyzed as individual case studies, using qualitative methods. Both clients and genetic professionals completed a post session questionnaire outlining their impressions of the session and the interaction, including how much time they talked about facts (education) and emotions. Also of interest was whether the psychosocial issues were better addressed if a greater rapport existed between clients and their counselor and how client's perceptions of the importance of cultural difference affected how they experienced genetic counseling.

\section{DECISION-MAKING ABOUT PRENATAL TESTING [81]}

\section{Muller and L. D. Cameron}

The University of Auckland, New Zealand

Understanding how future parents make choices about whether or not to undertake prenatal genetic testing is crucial to developing informational materials and support services. This study investigates the relative importance of emotional (e.g. worry) and cognitive (e.g. beliefs) variables in the decision-making process. 143 participants (age $M=28.66$ years, $S D=9.0$ years, 26 males) completed an anonymous, online survey which started with a scenario asking participants to imagine being under 12 weeks pregnant and having heard of a prenatal test able to detect the presence of a fatal, adult-onset condition responsible for neurological deterioration in the genetically affected individual. Participants completed measures assessing emotional and cognitive factors, and anticipated intentions to undergo testing; ratings ranged from -3 (not at all/strongly disagree) to +3 (very much/strongly agree). Anticipated intentions to obtain the prenatal test $(M=-1.00, S D=1.65)$ were associated with greater anticipated emotional distress $(r=.23)$, trust in the test's reliability $(r=.34)$, beliefs that others would expect one to get tested $(r=.40)$, motivation to comply with others' encouragement to get tested $(r=.37)$, views about the condition's impact on someone's life $(r=.23)$, lower dispositional optimism $(r$ $=-.16)$, religious convictions $(r=-.25)$, attitudes that reproductive technologies are useful $(r=.30)$ and pro-abortion attitudes for oneself $(r=$ 30). However, only Behavioral Beliefs $(M=.58, S D=1.11, \beta=.22, t=$ $2.39, p<.05)$ and Normative Beliefs $(M=3.08, S D=1.43, \beta=.25, t=$ $2.11, p<.05)$ independently predicted anticipated intentions and attitudes towards disabilities that may play important roles in decisions to use genetic prenatal testing.

\section{MYOCLONIC EPILEPSY IS ASSOCIATED WITH DELETIONS IN THE LONG ARM OF CHROMOSOME 5 [147]}

S. Nasioulas ${ }^{1,2,3}$,A. Boys', D. Bruno ${ }^{1,2}$, P. Lockhart ${ }^{2,3}$, H. Slater ${ }^{1,2}$, I. E. Scheffer ${ }^{2,4}$, and D. du Sart ${ }^{1,2}$

Victorian Clinical Genetics Services, Cyto-Molecular Diagnostics Research, Murdoch Childrens Research Institute, Royal Children's Hospital, Australia

2 Department of Paediatrics, University of Melbourne, Royal Children's Hospital, Australia ${ }^{3}$ Bruce Lefroy Laboratory, Murdoch Childrens Research Institute, Royal Children's Hospital, Australia

${ }^{4}$ Epilepsy Research Centre and Department of Medicine, University of Melbourne, Austin Health, Melbourne, Victoria, Australia

Myoclonic seizures are characterized by bilateral, single, or repetitive jerks, without loss of awareness. Myoclonic seizures are found in specific age-related epilepsy syndromes. One infantile onset epilepsy syndrome with myoclonic seizures called Dravet syndrome is associated with sodium channel gene (SCN1A) mutations in $80 \%$ cases. A common adolescent onset myoclonic epilepsy syndrome called juvenile myoclonic epilepsy has been associated with GABA subunit and chloride gene mutations in rare instances. We describe three unrelated patients showing subtle de novo deletions in the region of 5q14.2-q15 and a fourth patient with a deletion and an inversion within the same region. These patients have deletions ranging from $2.3 \mathrm{Mb}-8.8 \mathrm{Mb}$ with a common deletion segment of $1.3 \mathrm{Mb}$. Their phenotypes are complex with all four patients having myoclonic seizures and 3 had febrile seizures. We have used FISH, and SNP microarray analysis to map the deletions in detail. These analyses have shown that the common deleted region contains five genes in the $5 q 14.3$ region. In addition, expression microarray analysis has identified a further gene candidate that shows reduced expression without being part of the structural rearrangement. The candidate genes were screened in a cohort of 96 patients diagnosed with myoclonic epilepsy as the major clinical symptom. High Resolution Melt analysis and sequencing were used to identify point mutations and MLPA and Quantitative Real Time PCR were used to detect deletion mutations. The outcome of the results from this cohort of patients will be presented.

\section{CONSANGUINITY AND CONGENITAL ANOMALIES IN A HOSPITAL POPULATION [44]}

N. Nauman', S. Jalali' ${ }^{2}$ S. Jehan ${ }^{2}$, S.A.Shami ${ }^{2}$, R. Shaheen ${ }^{3}$, and R. Hamid ${ }^{3}$

Rawalpindi Medical College, Pakistan

2 Quaid-i-Azam University, Islamabad

FGSH Islamabad

Consanguinity is common in Pakistan. Autosomal recessive disorders are related to consanguinity and approximately $30 \%$ of sporadic cases of mental retardation, congenital anomalies and dysmorphism may have an autosomal recessive etiology with risks of recurrence in future pregnancies. The aims of this study are to determine the number of consanguineous unions in parents of children born with congenital anomalies. The study population was comprised of 70 pregnant women coming for delivery/ termination to the Obstetrics Department, Federal Services Hospital, Islamabad, Jan-June 2004. Seventy percent of couples were consanguineous, and of these $60 \%$ were first cousins. The majority $(58.56 \%)$ of congenital anomalies were neural tube defects, including anencephaly, spina bifida and meningocele. Most congenital anomalies were autosomal recessive and multifactorial. $84 \%$ of patients were from urban areas and $16 \%$ from rural areas, the majority of patients were illiterate or had limited education and most had low socioeconomic status. The congenital anomalies encountered were autosomal recessive with a risk of recurrence in subsequent pregnancies and genetic counseling could be beneficial for consanguineous couples. There is also a need to educate the public on the possible harmful effects of inbreeding, especially in developing countries with high rates of consanguinity and limited financial resources.

\section{ROLE OF HAND2 IN A FAMILIAL CASE OF CONGENITAL} HEART DISEASE [3]

G. Nemer, R. Tanos, F. Hariri, M. Nehme, and F. Bitar

American University of Beirut, Lebanon

Members of the basic helix-loop-helix (bHLH) transcription factor family are known to regulate the specification and differentiation of many cell lineages during embryogenesis. The bHLH proteins Hand 1 and 2 are expressed in the cardiac tissue and knock-out studies for these 2 genes lead to embryonic lethality. We aim at linking mutations in Hand 2 to patients with congenital heart disease (CHD) in Lebanon. By direct DNA sequencing of 44 patients, we showed that a heterozygous mutation G205V is linked to patients with CHD in one family with different CHD phenotypes.Biochemical analysis identified members of the T-box family of transcription factors as partners for Hand2 and that the G205V mutation abrogates the interaction with Tbx 5 but not Tbx20. This is the first evidence of the implication of the Hand 2 in CHD and identifies a novel transcriptional circuit for heart development and disease.

\section{MULTICOLOUR FLUORESCENCE IN-SITU HYBRIDISATION OF COMPLEX KARYOTYPES- SHOULD IT BE OFFERED ROUTINELY? [143]}

\section{Nicola and J. Suttle}

SA Pathology, IMVS, Australia

Detailed characterisation of karyotypic abnormalities such as markers, derivative chromosomes and complex rearrangements are limited by chromosomal morphology, banding resolution and by similarities in banding patterns. Elucidation of complex karyotypes is labour intensive and can be challenging for even the most experienced cytogeneticist. The selection of Fluorescence In-Situ Hybridisation (FISH) probes to delineate abnormalities is a matter of trial and error, sometimes fails to yield a worthwhile result. These problems and the time constraints of a busy cytogenetic laboratory may therefore hamper accurate characterisation of cytogenetic abnormalities. Multicolour FISH (M-FISH) has revolutionised cytogenetic analysis by allowing for the effortless interpretation of complex rearrangements even in the presence of poor chromosome morphology. Relatively inexperienced operators can interpret results and the limitations of MFISH are few. We present 3 cases in which M-FISH was pivotal in interpreting the karyotypes: (1) a solid tumour with poor morphology chromosomes; (2) an AML with double minutes and (3) a solid tumour with a complex karyotype. Apart from the inevitable time delay due to 
extended hybridisation times, M-FISH overcame the inherent problems associated with G-banded chromosomes and allowed a more complete picture of the karyotype. We propose to offer an M-FISH service in cases where there is a complex karyotype or the presence of unidentified chromosomal material. The increased karyotypic accuracy will allow greater insight into assigning clinical significance to our findings and, in some cases, will increase the sensitivity of subsequent monitoring of minimal residual disease by FISH.

\section{ARRAY CGH STUDY OF PATIENTS WITH APPARENTLY BALANCED TRANSLOCATIONS: CRYPTIC ABNORMALITIES AND UNEXPECTED FINDINGS [216]}

J. Nicholl',W.Waters', E. Haan $^{2}$, J. Liebelt', E.Thompson², D. Bratkovic', and S.Yu' Cytogenetics Unit' and South Australian Clinical Genetics Service ${ }^{2}$, SA Pathology, Women's and Children's Hospital, North Adelaide, Australia

Apparently balanced chromosome rearrangements in patients with phenotypic abnormalities represent a challenge for genetic counselling. Utilisation of array CGH has shown that many translocations have cryptic deletions at the breakpoints. Identification of the genes within these deleted regions may aid in providing an understanding of the molecular basis of the phenotype. Array CGH studies on eight patients with idiopathic intellectual disability and dysmorphic features who have apparently balanced rearrangements, detected cryptic deletions at the breakpoints in three patients. Patient 1 , with a complex $\mathrm{t}(1 ; 7 ; 2 ; 16)$, had an interstitial deletion at the $7 q$ breakpoint. Patient 2 with an ins(11;16), had a deletion at 11q. Patient 3, with two apparently balanced translocations $t(6 ; 14)$ (mat) and $\mathrm{t}(5 ; 12)$ (mat), had two deletions. In a fourth patient with a complex translocation $\mathrm{t}(2 ; 8 ; 10)$, two interstitial deletions were identified on chromosome 11q. The first at 11q22-23, 4.61-6.23 Mb in size, contains a number of genes known to associate with human diseases. The second at $11 \mathrm{q} 25$ is likely to be nonpathogenic and has been reported in at least three normal individuals. In summary: $50 \%$ of apparently balanced rearrangements were unbalanced, the imbalance not necessarily at the putative breakpoints.

\section{A PATHOLOGIC EXPANSION IN FMR1 5' UTR CGG REPEATS PLUS RECOMBINATION AND MOSAICISM IN A FAMILY WITH FRAGILE X SYNDROME [5]}

M. Noruzinia and M. Keyhanee

Department of Medical Genetics, Tarbiat Modares University, Tehran, Iran

In this article we present a family with several male members with mental retardation corresponding to a heritable disease with $\mathrm{X}$-linked recessive inheritance. The phenotype corresponded to Fragile X syndrome. However, the phenotypes were different in the two patients. We developed four PCR based methods to detect full mutation in these patients. This method confirmed Fragile X syndrome in these patients. However in the patient with milder phenotype a mosaic form of full and permutation was detected that corresponded to clinical findings. STR marker analysis showed the presence of different STR alleles in these two patients. This finding could be explained by occurrence of a recombination in the region. This is the first case of mosaic Fragile X syndrome reported in an Iranian patient. In this case report we present several PCR based triplet repeat mutation detection techniques that can be implemented in molecular laboratories.

\section{FAMILY HISTORY OF CHRONIC DISEASES IN MEXICO: A GENOMIC TOOL FOR RISK ESTABLISHMENT IN PUBLIC HEALTH [209]}

P. F. Oliva-Sanchez', E.Velasco-Mondragon ${ }^{2}$, R. Lopez-Ridaura ${ }^{3}$, and G. Jimenez-Sanchez'

'National Institute of Genomic Medicine, Mexico

School of Public Health and Policy, Morgan State University, Baltimore, Maryland

National Institute of Public Health, Mexico

This study evaluated the association between chronic diseases, such as diabetes type 2 (DT2), hypertension (HTA) and the metabolic syndrome (SM), with family history (FH) in the Mexican adult population. A statistical analysis of the National Health Survey of 2000 (ENSA 2000) was performed. This survey was implemented by the National Institute of Public Health and the Mexican Ministry of Health, and consisted of interviews with a random sample of adults over 20 years of age in Mexico. We evaluated the association between chronic diseases and family history and calculated adjusted and non-adjusted odds ratios (OR) using logistic regression. The sample size was $n=45294$. 7.5\% (3 334) of participants reported suffering from DT2, 31.6\% (14 004) from HTA and 2.5\% (696) from SM. We observed that those individuals who reported a history of DT2 in both parents had a greater probability of suffering from DT2 that those with no family history (OR 5.1, $p<.0001$ ). People with SM were also more likely to have parents who suffered from DT2 that those who did not have SM (OR 9.15, $p<.0001)$. When we performed a stratified analysis based on BMI, the association between DT2 and FH was the same for all groups (e.g., normal, overweight and obese) We consider that $\mathrm{FH}$ is a genomic tool that serves to detect groups of greater vulnerability to disease.

\section{ANALYSIS OF CANDIDATE GENE POLYMORPHISMS AND ENDOMETRIAL CANCER RISK [156]}

\section{T. O'Mara ${ }^{1,2}$, K. Ferguson ${ }^{2}$, P.Webb' ${ }^{2}$, and A. Spurdle ${ }^{2}$}

School of Life Sciences and Cells and Tissue Domain, Institute of Health and Biomedical Innovation, Queensland University of Technology, Australia

Genetics and Population Health Division, Queensland Institute of Medical Research, Australia

Endometrial cancer (cancer of the uterine corpus) is the most common malignancy of the female genital tract. Each year, it is estimated there are almost 200,000 cases diagnosed worldwide, and an estimated 50,000 women will die from this disease. From the limited number of populationbased studies performed, it is known that family history of endometrial cancer among first-degree relatives is associated with up to 3-fold risk of endometrial cancer, suggesting a genetic component for this disease. Genetic polymorphisms in functionally critical genes have been suggested as risk factors for the development of a variety of cancers, including endometrial cancer. There have been many studies performed investigating endometrial cancer risk and survival with various single nucleotide polymorphisms (SNPs), including SNPs harbored by genes from the estrogen metabolism pathway (CYP1A, CYP17, CYP19A1 and SULT1A1), mismatch repair genes (MLH1, MSH2, MSH6) and steroid hormone receptors (ESR1 and PGR). We have prioritized SNPs that have previously been reported to be associated with endometrial cancer, as well as candidate SNPs from relevant pathways and genes of suspected functional significance. These have been genotyped in approximately 1400 cases and 1400 controls from the population-based Australian National Endometrial Cancer Study using the Sequenom MassArray platform and assessed for association with endometrial cancer predisposition. The results from the analysis of this study will be presented.

\section{COUNSELLING AND CONSENT: THE EXPERIENCES OF PEOPLE WHO HAVE CONSENTED TO TESTING OF STORED TUMOUR MATERIAL FOR HEREDITARY NON POLYPOSIS COLORECTAL CANCER PREDISPOSITION [61]}

A. Opat', F. Macrae' ${ }^{2}$, and C. Gaff ${ }^{3}$

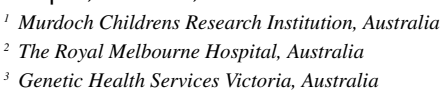

This study aimed to inform service provision to individuals who may have Hereditary Non Polyposis Colorectal Cancer (HNPCC). Testing of stored tumour material for the mismatch repair defects typical of HNPCC has been found to be an effective and economic method of identifying those at higher risk of having a mutation and therefore eligible for germline genetic testing. Although tumor testing is widely used, guidelines for pretest counseling and consent have not been defined. This study explores the experiences of those who have been asked to consent to tumor testing, either after genetic counselling or by a relative (no professional counseling). People who had given consent $(n=17)$ were recruited through a Familial Cancer Centre and in-depth interviews conducted Decisions to undergo testing were made very quickly. Factors influencing decisions included desire to help family and availability of effective prevention strategies. Participants informed rather than consulted family members of decisions to consent to tumor testing. Participants that consented in the clinic understood basic concepts of tumour testing rather than details. Participants consenting out of clinic $(n=8)$ received less information although most participants expressed satisfaction. Three participants had no memory of the test. Understanding of results was variable. Many expressed 'relief' at being 'negative' and there was no understanding of distinction between tumour and germline testing. Many participants expressed gratitude for the opportunity to participate in testing and for dietary and surveillance advice received through the clinic. Implications for practice and theoretical implications in relation to informed consent and decision making theories will be discussed.

\section{ATYPICAL CHRONIC LYMPHOCYTIC LEUKAEMIA AND NON-HODGKIN'S LYMPHOMA WITH THE T(14;19)(032;013)} AND REARRANGEMENT OF BCL3 [184]

J. O'Reilly, P. Cannell, and J. Cooney

Department of Haematology, Royal Perth Hospital, Australia

Cytogenetics is playing a greater role in the classification and management of lymphoproliferative disorders. The relatively rare $t(14 ; 19)(q 32 ; q 13)$ was first described in cases of chronic lymphocytic leukaemia and is now reported in a heterogeneous group of B-cell neoplasms. When classified as 
CLL the patients have atypical morphology and immunophenotype and low CLL scores presenting a diagnostic dilemma. The distinction of patients with $\mathrm{t}(14 ; 19)$ between CLL and NHL is somewhat influenced by the type of additional chromosomal abnormalities present in the karyotype, with trisomy 12 closely associated with CLL. The result of this translocation is the deregulation of the BCL3 gene due to it's juxtapositioning with regulative elements of the IGH gene. We report two cases of NHL/CLL with $\mathrm{t}(14 ; 19)(\mathrm{q} 32 ; \mathrm{q} 13)$ both presenting with a raised white cell count and abnormal lymphocytosis with splenomegaly and one case having generalised lymphadenopathy. Involvement of the BCL3 gene in both cases was demonstrated using a BCL3 breakapart probe. Although both patients presented with this rearrangement, one was classified as atypical chronic lymphocytic leukaemia and the other as non-Hodgkin's lymphoma. We review the available information including the suggestion of $t(14 ; 19)$ forming a specific category within the WHO classification of lymphoid neoplasms and discuss the prognosis and response to therapy.

\section{A LONGITUDINAL SURVEY OF AUTOSOMAL DOMINANT POLYCYSTIC KIDNEY DISEASE [211]}

N. Pachter', S. Ranasinha ${ }^{2}$, M. Jenkins ${ }^{2}$, R. Gibson ${ }^{3}$, R.Walker ${ }^{3}$, D. Ravine ${ }^{4}$, and I.Winship 2,3

'Victorian Clinical Genetics Service, Australia

${ }^{2}$ University of Melbourne, Australia

Royal Melbourne Hospital, Australia

${ }^{4}$ Western Australian Institute of Medical Research, Australia

Longitudinal studies of disease progression in autosomal dominant polycystic kidney disease (ADPKD) are currently limited to periods up to 7 years among adults and 15 years among cases diagnosed in childhood. The US-based observational study of patients with non-azotemic ADPKD (Consortium of Radiologic Imaging Studies of Polycystic Kidney Disease), designed to ascertain determinants influencing disease progression, is currently limited to observations over a three-year period, with many conclusions currently based on retrospective extrapolations (Grantham et al. 2008). Retention of records of a survey conducted during 1988-1991 among ADPKD families in Victoria offered the opportunity for a 17-19 year follow-up of affected subjects. A follow-up survey of cases newly diagnosed as a result of the earlier survey has been conducted with the aim of seeking further insight into the factors influencing disease progression. Comparison between baseline and follow-up clinical data revealed a significant increase in average renal length and decrease in glomerular filtration rate (GFR) reflecting progression of disease. Predictors at baseline of disease progression included average renal length, mean arterial blood pressure and GFR. There was no evidence that gender influenced the course of renal disease. Preliminary follow-up assessment of subjects contributing to the Victorian ADPKD study reveals the extent of the opportunity available to contribute to international efforts to characterise the long-term factors contributing to disease-progression among patients with ADPKD.

\section{THE LEGALITY OF GENE PATENTS [197]}

\section{Palombi}

The Centre for the Governance of Knowledge and Development, The Australian National University, Australia

Thousands of patents have been granted around the world creating a patchwork of patent monopolies over genetic materials. Patentees in Australia (and this is true in most countries) are completely within their legal rights to impose whatever conditions they believe are appropriate in the exploitation of the genetic materials that come within the scope of these patent monopolies. The consequences are significant. In Australia (and also in other countries), patentees, or those that have been assigned their legal rights, are using those powers to control research and development of genetic tests. They have also threatened to bring legal proceedings to restrain the use of non-licensed genetic tests performed by publicly funded laboratories. That said, no patent wherever granted is guaranteed validity. Indeed, apart from in the United States, no granted patent comes with any presumption of validity. Unfortunately, these patents have escaped critical legal review. The result is a misconception, particularly within scientific and medical communities, that such patents need to be tolerated and respected. Apart from imposing additional burdens on scientific researchers in the form of licensing costs, the results of their research can be made the subject of contractual obligations that effectively give to patentees the right to control any intellectual property that is developed. This paper explores the legality of gene patents and patents over genetic tests dealing with two patentability thresholds - patentable subject matter and inventive step. It not only questions their validity, but the suitability of the patent system in the field of genetics.
EVALUATION OF THE EFFICACY OF BIOCHEMICAL AND CLINICAL SELECTION CRITERIA FOR GENETIC TESTING OF SUSPECTED HNPCC PATIENTS IN A CLINICAL SETTING [42]

D. Parker', A. Bremner', L. Schofield ${ }^{2}$, L. Colvin-james ${ }^{2}$, and S. Townshend ${ }^{2}$

'School of Population Health, University of Western Australia, Australia

${ }^{2}$ Genetic Service of Western Australia, King Edward Memorial Hospital, Perth, Australia

This was a retrospective evaluation of the effectiveness of immunohistochemical (IHC), miscrosatellite instability (MSI) screening and clinical criteria at finding mutation carriers for one of the 4 HNPCC genes in a series of potential HNPCC families. We compared all the criteria for each family tested for any of the 4 mismatch repair genes. Retrospective examination of 212 patient files referred to Genetic Services of Western Australia (GSWA) identified pathogenic mutations in 71 (35 MSH2, 26 MLH1, 6 MSH6, 4 PMS2) (33.5\%) individuals. Although $82.1 \%$ patients fulfilled some form of clinical criteria, only $25.5 \%$ met original Amsterdam criteria. Of the patients who had IHC performed $70.1 \%$ had a high-risk for the disease and those that underwent MSI, 63.7\% had a high-risk result. Either screening technique (MSI or IHC) is more effective than clinical criteria in selecting mutation search. In this cohort IHC was identified as the best strategy for detecting patients with mutations in MLH1 and MSH2, however MSI is the more effective method for MSH6 and PMS2 mutation detection. This implies a combined approach will yield best results.

SMITH-MAGENIS SYNDROME: NEW PRENATAL FINDINGS IN THE 1ST AND 2ND TRIMESTER [185]

E. Peach and S. Sinnott

Specialised obstetrics + gynaecological imaging (so+gi), Brisbane, Queensland, Australia

Smith-Magenis syndrome is a microdeletion syndrome characterised by distinctive facial features (including midfacial hypoplasia and a broad nasal bridge), developmental delay, mild to moderate mental retardation, and behavioral abnormalities. The majority of patients are diagnosed during childhood due to their complex behavior and sleep disturbances. To date there have been two reported cases diagnosed during the prenatal period. Both were diagnosed following amniocenteses performed after detection of multiple congenital anomalies on second trimester ultrasound. We report a case of Smith-Magenis syndrome diagnosed prenatally in which the first trimester screening results were low $(\mathrm{T} 21=1 / 1058$ and $\mathrm{T} 13 / 18=1 / 1158)$ and the only abnormal findings on ultrasound were an absent nasal bone at 12 and $1 / 7$ weeks and a $4 \mathrm{~mm}$ isolated pericardial effusion detected at 20 and $1 / 7$ weeks. Neither of these findings has previously been reported in association with Smith-Magenis syndrome. Absent nasal bone in the first trimester screen has been associated with an increased risk for aneuploidy (specifically T21, T13, T18 and Turner syndrome). However, there are a number of other genetic syndromes that have midfacial hypoplasia as a characteristic feature. In cases of isolated absent nasal bone in the 1st trimester, it can be difficult to counsel patients regarding potential risks beyond aneuploidy. Since examination of the nasal bone has become more frequent, there is potential for earlier diagnosis of other genetic syndromes, such as the one presented.

\section{OLIGODENDROGLIOMA AND ASTROCYTOMA FLUORESCENCE IN SITU HYBRIDISATION DETECTION - THE WESTERN AUSTRALIAN EXPERIENCE [85]}

\section{J. Peverall, F. Price and E. Baker}

Cytogenetics Department, PathWest, King Edward Memorial Hospital, Subiaco, Western Australia, Australia

Oligodendrogliomas, astrocytomas and mixed oligoastrocytomas are types of diffuse gliomas, the most common primary cancer of the central nervous system. The loss of genetic material from chromosomes $1 p$ and $19 q$ is strongly associated with oligodendroglioma histology and the amplification of the EGFR gene is usually a hallmark of astrocytoma. Fluorescence in situ hybridization (FISH) analysis to detect these genetic changes can be a useful diagnostic tool as distinguishing glial subtypes based on morphology can sometimes be difficult. Furthermore, positive FISH results have been found to be associated with therapeutic response and patient survival. We present FISH experience in the detection of $1 \mathrm{p} / 19 \mathrm{q}$ deletion and EGFR amplification in formalin-fixed, paraffin embedded sections of excised brain tissue in our laboratory. From 2006 to 2008, over 130 cases were analyzed using in-house FISH probes to chromosomes $1 \mathrm{p} 36$ and $19 \mathrm{q} 13$, and a commercial EGFR gene. Interesting combinations of abnormal results were found involving elements of both oligodendroglioma and astrocytoma. The implications for patients and eventual outcomes of treatment are discussed. 
FAMILIAL HYPERCHOLESTEROLAEMIA (FH) PILOT CASCADE SCREENING PROJECT [174]

S. Poke, G.Watts, S. Maxwell, K. Brameld, and P. O'Leary

Office of Population Health Genomics, Department of Health WA, on behalf of FHWA, Australia

Familial hypercholesterolaemia (FH) is an inherited disorder of cholesterol metabolism leading to premature coronary heart disease (CHD). The early diagnosis and treatment of $\mathrm{FH}$ can delay or prevent the onset of CHD. The FHWA program has three components: an education program for general practitioners, model of care, and support for families with $\mathrm{FH}$. Feedback from the education program has helped to refine and target the information required to identify and manage FH cases. The model of care that has been developed for screening adults and children at risk of $\mathrm{FH}$ provides clinical pathways for investigation and monitoring index cases and for involving families in cascade screening. The cases were identified initially based on phenotype and have since undergone genotypic testing. Of the 121 index cases that have been assessed, 91 relatives have been identified by cascade screening as having FH. The availability of genotyping has simplified the process of identifying affected relatives. A family support organization has been established to improve information, communication and support services for families with FH. The outcomes from this pilot program provide a service framework, support for $\mathrm{FH}$ families and a practical examination of issues related to targeted genetic screening, sharing family health information and the translation of research into improvements in community health.

EXTENDED FAMILY STUDY OF AN INHERITED INS(3;7)(P?21;011.23) INVOLVING THE W.S SYNDROME REGION, DETECTED BY FLUORESCENT IN SITU HYBRIDISATION [76]

F. Price, J. Peverall, E. Baker, and T. Bowen

Cytogenetics Department, PathWest, King Edward Memorial Hospital, Australia

An extended family study of the inheritance of an ins(3;7) and the derivative chromosomes resulting from it. The insertion involves the W.s Syndrome locus (containing the genes ELN and LIMK1), and is inserted into $\mathrm{p}$ ?21 on the short arm of chromosome 3 . There are two members of the family that are balanced carriers of the insertion, and all possible combinations of inheritance of the derivative chromosomes are present in various members of the family. Seven members of the family have inherited the derivative del(7), and one family member has the der(3)ins( $3 ; 7)$, making them effectively trisomic for the W.s syndrome region. The insertion and the resulting derivative chromosomes are not visible by conventional cytogenetics, and Fluorescence In Situ Hybridisation (FISH) was used to identify the status of the individuals. A commercial W.s Syndrome probe, spanning ELN and LIMK1, was used on all family members to determine the presence of the ins $(3 ; 7)$ or derivative chromosomes. Furthermore, an inhouse produced W.s Syndrome probe set, containing 4 individual probes and spanning a larger area of $7 \mathrm{q} 11.23$, was used on one family member to determine the extent of the rearrangement. It was found that only 1 of the probes, that containing ELN and LIMK1, was involved in the rearrangement (and deleted in family members with the $\operatorname{del}(7)$ ), thus corresponding to a relatively mild phenotype. A larger deletion of $7 \mathrm{q} 11.23$ is necessary for the full W.s Syndrome syndrome cognitive and behavioral profile.

\section{OVER-EXPRESSION OF DSCR1/RCAN1 IS ASSOCIATED WITH DOWN SYNDROME-RELATED BRAIN ABNORMALITIES [115]}

M. Pritchard', D. Dubach', H. Parkington ${ }^{2}$, H. Coleman², D. Keating ${ }^{3}$, D. Bowser ${ }^{4,5}$, and D. Finkelstein ${ }^{5}$

Dept Biochemistry and Molecular Biology, Monash University, Australia

Dept Physiology, Monash University, Australia

Molecular and Cellular Neuroscience, Flinders University, Australia

The Howard Florey Institute, University of Melbourne, Australia

Mental Health Research Institute, Parkville, Australia.

Numerous chromosome 21 genes are over-expressed in Down syndrome (trisomy 21). The challenge is to determine which genes contribute to which specific phenotypes. Our efforts to identify genes on human chromosome 21 with the potential to cause the brain anomalies observed in Down syndrome, led to the discovery of a gene, Down syndrome candidate region-1 (DSCR1) now renamed RCAN1 (Regulator of Calcineurin-1). In order to understand the consequences of RCAN1 over-expression in the brain we generated Rcan1 transgenic mice and have begun to investigate CNS morphology and function. We found that over-expression of Rcan1 leads to a disproportionately small hippocampus and that the hippocampal pyramidal neurones display a significant reduction in post-synaptic dendritic spine density. Probably as a consequence of this, LTP, a measure of synaptic function, is reduced in Rcanl transgenic mice. We have also found using chromaffin cells as a neuronal cell model, that there is a disruption in vesicle trafficking in Rcanl transgenics, and we therefore expect to find a similar defect in neurones using synaptopHluorin as an optical tracer to track the net balance of exocytosis and endocytosis at nerve terminals. These results reveal that up-regulation of Rcanl leads to some of the aberrant brain phenotypes associated with Down syndrome.

\section{A MUTANT SKELETAL MUSCLE ALPHA-ACTIN GENE, FUSED TO EGFP PRODUCES A UNIQUE MYOPATHIC MOUSE MODEL [191] \\ G. Ravenscroft ${ }^{1,2}$, C. Jackaman', S. Marston ${ }^{3}$, J. Papadimitriou4, K. E. Davies ${ }^{5}$, N. G. Laing', and K. J. Nowak' \\ Centre for Medical Research, University of Western Australia, and Western Australian Institute for Medical Research, Australia \\ Department of Physiology, University of Western Australia, Australia \\ Cardiac Medicine, National Heart and Lung Institute, Imperial College London, United Kingdom \\ ${ }^{4}$ Department of Physiology, Anatomy and Genetics, University of Oxford, Oxford, United Kingdom \\ School of Pathology and Laboratory Medicine, University of Western Australia, Australia}

Mutations in the skeletal muscle alpha-actin gene (ACTA1) cause distinct congenital myopathies. A transgenic mouse model was created using a nemaline myopathy mutant (D286G) human ACTA 1 cDNA fused to enhanced green fluorescent protein (EGFP), both driven by the human skeletal muscle alpha-actin promoter. Skeletal muscle from the transgenic animals was analysed by histology, fluorescent microscopy, immunohistochemistry, western blot analysis, electron microscopy, physiological methods and motility assays. Transgenic mice have fluorescent green skeletal muscle due to the expressed ACTA1(D286G)-EGFP fusion protein. Fluorescent green aggregates disrupting the Z-lines and co-staining for alpha-actinin and myotilin are present in the muscles. Cultured differentiated primary myoblasts and flexor digitorum brevis myofibres exhibit multiple fluorescent green rod-like structures. Electron dense osmophilic bodies are visible by electron microscopy. At one month of age many internal nuclei are visible in certain muscles, but are less prominent at later stages. Ringbinden are noticeable by histology, immunohistochemistry and electron microscopy in some muscles at multiple ages. Interestingly, the pattern of muscle involvement and the pathological features change markedly with age. Purification of the mutant actin-EGFP protein indicates that the majority is not incorporated into sarcomeres, however, by a motility assay, actin filaments from these mice have a $19 \%$ faster sliding speed than wild-type alpha-actin. Skinned extensor digitorum longus muscle fibres are not as weak as expected from the observed pathology. This transgenic mouse is a good model of ACTA1 disease and due to the expression of the ACTA1(D286G)-EGFP fusion protein, is a valuable tool for studying disease pathogenesis.

\section{JEWISH GENETIC SCREENING PROGRAM COMES}

\section{OF AGE [24]}

D. Redelman,A. Proos, and L. Burnett

Pacific Laboratory Medicine Services (PaLMS), Royal North Shore Hospital, Sydney, Australia

Australian community testing programs for Tay-Sachs disease (TSD) carrier testing began in Sydney in 1992. In 1995, the program was extended into senior High Schools. The program has two major routes of access: testing free of charge in year 11, and fee-for-service testing at an outreach centre in Sydney (as well as through general practitioners and specialist referrals). In the schools program, for the periods 1995-6 and 2001-4, only TSD was offered, while for 1997-2000, TSD and cystic fibrosis (CF) were offered. Since 2005, TSD, CF and 3 other genetic tests have been offered. In the outreach clinic, testing for 9 genetic conditions are currently available. All clients provide informed consent prior to testing. In the schools program, a pre-test compulsory education session is held; previous studies have demonstrated retention of knowledge for periods in excess of three years. In the outreach setting, printed information and face-to-face professional advice are offered. In spite of the above, we encounter enquiries from clients who have previously been offered testing, seeking additional information or interpretation. Enquiries include details on accessing results, replacement of lost results, or enquiries for further testing of additional genetic conditions previously not available. Previous surveys indicate choosing to be tested and positive carrier status are good predictors of retention of knowledge, demonstrating personal significance. This preliminary survey will explore how well knowledge is retained until a time relevant for its use (marriage/reproduction). 


\section{A FAMILY HISTORY SCREENING STUDY IN GENERAL PRACTICE - THE STORY SO FAR [162]}

\author{
G. Reid', F.Walter', J. Brisbane', and J. Emery ${ }^{1,2}$ \\ General Practice, School of Primary, Aboriginal and Rural Health Care, The University of \\ Western Australia, Australia \\ General Practice and Primary Care Research Unit, Institute of Public Health, University of \\ Cambridge, United Kingdom \\ Office of Population Health Genomics, Health Department of Health Western Australia
}

Evidence of the clinical utility of the family history as a screening tool in general practice is limited. This study aims to determine the performance of a family history screening instrument in general practice for the assessment and management of chronic disease risk. Informed by a systematic review of family history questionnaires, a 15-item Family History Screening Instrument (FHSI) was developed. Three metropolitan and 1 outer-metropolitan general practices participated in the study. Inclusion eligibility for practices was based on the practice having an up-to-date electronic register of patients that could be searched for recently attending patients and those aged 20 to 50 years of age. Each practice staggered the mail out of 500 invitations to a randomized subset of regularly attending, 20- to 50-year-old patients. Participants attended an appointment with a genetic counselor at their general practice. They completed a self-reported health questionnaire and the FHSI. A three generational pedigree was also collected for each participant. Participants were posted a 1-week and 3month follow-up self-reported health questionnaire to complete. Accounting for returned mail, 1838 invitations were posted out from the 4 practices in a 3 and half month period. Of these, 104 participated in the study (5.7\% response rate). Up-to-date details of disease prevalence, risk stratification and psychological measures will be described. Despite a relatively low response rate, the overall number of respondents in a 3.5 month period allows a forecast of another 12 months to obtain the target number of 500 participants.

\section{AUTOZYGOSITY MAPPING REVEALS YARS2 AS A CAUSE OF MLASA: MYOPATHY, LACTIC ACIDOSIS AND SIDEROBLASTIC ANAEMIA [188]}

L. Riley', P. Hickey ${ }^{2}$, S. Cooper ${ }^{3,4}$, D. Thorburn ${ }^{5}$, A. Compton ${ }^{5}$, M. Ryan ${ }^{6}$,

E. de Leon', M. Bahlo' ${ }^{2}$ and J. Christodoulou ${ }^{1,4}$

Genetic Metabolic Disorders Research Unit, Children's Hospital at Westmead, Sydney, Australia

${ }^{2}$ Bioinformatics Division, The Walter \& Eliza Hall Institute of Medical Research, Melbourne, Australia

${ }^{3}$ Neuorgenetics Research Unit, Children's Hospital at Westmead, Sydney, Australia

${ }^{4}$ Discipline of Paediatrics \& Child Health, University of Sydney, Sydney, Australia ${ }^{5}$ Murdoch Children's Research Institute, Royal Children's Hospital, Melbourne, Australia

${ }^{6}$ Department of Biochemistry, LaTrobe University, Melbourne, Australia

Mitochondrial respiratory chain disorders are a heterogeneous group of disorders due to mutations in either mitochondrial or nuclear encoded genes, with marked clinical inter- and intrafamilial phenotypic variability. Identification of causative mutations is important not only for diagnosis and genetic counseling, but also to understand the pathogenesis of these disorders. We studied a family where consanguineous parents have had three healthy children and two children with persistent lactic acidemia, progressive skeletal myopathy, transfusion-dependent sideroblastic anaemia and deficiencies in mitochondrial complex I \& IV activity in skeletal muscle. DNA mutation analysis excluded PUS1 as the causative gene. We undertook multipoint autozygosity mapping of this family using the 250K NspI Affymetrix Human GeneChip Array. Six regions of interest with a peak LOD score of 2.0 were identified. Those regions whose haplotypes fit our hypothesis of a single ancestral mutation underwent in silico analysis using the MitoCarta database to identify 28 candidate genes encoding proteins with mitochondrial functions. DNA sequencing revealed a homozygous missense mutation in the two affected children in one of the candidate genes, YARS2, which encodes mitochondrial tyrosyltRNA synthetase (mt-TyrRS). The same missense mutation was also found in homozygous form in an apparently unrelated individual with the same ethnicity, clinical presentation and enzyme defect but was absent from 50 ethnically matched control individuals. mt-TyrRS is responsible for attaching Tyr to its cognate tRNA for incorporation into mitochondrial proteins. Studies were undertaken to determine the expression level, stability and functionality of the mutant protein and its effect on mitochondrial protein synthesis.

\section{FRAGILE X SYNDROME: THE MORE WE KNOW, THE HARDER IT GETS [189]}

\section{Rogers, G. Turner, K. Frumar, and M. Field}

GOLD Service, Center for Genetics Education, NSW Health, Australia

The GOLD Service has been providing genetic counseling to individuals who have a family history of fragile $\mathrm{X}$ syndrome for over 20 years. Counseling has become more difficult with the discovery of POFS,
FXTAS, individuals being identified in the 'grey zone' (intermediate repeat size) and some individuals in the premutation range presenting with learning and or behavioral problems. We will present three individual case studies that highlight some of these issues and examine options available for counseling families. Case 1: A 17-year-old girl, known to be an obligate carrier but not tested. Father tested previously but had not informed his three daughters. Daughter informed of family history when parents were aware she was pregnant. Susan tested for carrier status and had a CVS. Both Susan and foetus showed a full mutation result. Case 2: A mother with a known premutation insisting that her 8-year-old son of normal intelligence be tested. Case 3: 35-year-old female premutation carrier wanting to have a child but experiencing POFS. Client considering freezing her eggs or having donor sperm and freezing embryo.

\section{MIXED MESSAGES: WHAT DO INTERPRETERS SAY WHEN} THERE ARE NO WORDS? [7]

M. Ross and P. James

Victorian Clinical Genetics Services, Interpreters, Southern Health, Monash Medical Centre, Melbourne, Australia

Monash Medical Centre is a busy hospital serving a multicultural population in excess of one million people. Many clients are young refugees and couples keen to have children now they have reached Australian 'safety'. All pregnant women are offered serum screening for Down syndrome, Trisomy 18 and neural tube defects. Women receiving an increased risk for any of these conditions are referred for genetic counseling to discuss their risk, the condition in question, and their choices. Interpreters greatly assist our efforts to provide genetic counseling and choices to these migrant couples, but it has become clear that there are times when the clients simply do not understand what the central issues under discussion are. This raises concerns that the decisions made may not have a sound base. Terms such as risk, chance, chromosomes, genes, Down syndrome and spina bifida are a mystery to some. In order to improve genetic counseling practice, interviews were conducted with hospital interpreters to elicit what is said, especially when there is not a corresponding word in their given language. Interviews consisted of standard questions as well as an exploration of generally held beliefs about inheritance, and how decisions are made in the family culture. A review of the questionnaire and the outcomes of these interviews will be discussed.

\section{PRENATAL ANEUPLOIDY TESTING: THE PITFALLS OF FISH:} LESSONS LEARNED IN GENETIC COUNSELLING [4]

M. Ross and G. McGillivray

Genetic Health Services Victoria, Monash Medical Centre, Clayton Victoria, Australia

In Victoria Australia, all pregnant women are offered serum screening to identify those fetuses that are considered to be at an increased risk for Trisomy 21, Trisomy 18 and Neural Tube defects. Women returning an increased risk may be counselled by their general practitioner, obstetrician, midwife or genetic counsellor, and are offered diagnostic testing. Many choose to have a 5-Probe FISH analysis as part of the diagnostic testing. I present a number of women who received a normal FISH result from diagnostic testing, believing that this was the complete result. They were later informed that the long-term karyotype was abnormal - an event that caused distress and confusion. I discuss their varied reactions and emotions in response to this 'changed' result, and how they addressed the unexpected and difficult decision-making that followed. Examination of these cases highlights the need to exercise great care when giving and discussing prenatal FISH results in the setting of increased risk for aneuploidy via serum screening. Clearer information about common and rare aneuploidies, given when the test is offered, may alleviate misunderstandings but does little to reduce anxiety. The cases also demonstrate a need to provide ongoing education for health professionals who offer these tests to their clients, often also believing that a normal FISH result is an accurate description of the final result.

\section{UPDATING FAMILIAL CANCER RESOURCES FOR} CONSUMERS: AN EVIDENCE BASED APPROACH [10]

D. Ross', K. Barlow-Stewart', K. Dunlop', B. Meiser ${ }^{2}$, and K. Strong ${ }^{2}$

Centre for Genetics Education. NSW Health, Australia

2 Psychosocial Research Group, Department of Oncology, Prince of Wales Hospital, Sydney, Australia

Given the rapid progress in the field of cancer genetics, consumer focused education materials addressing topics relevant to families affected by hereditary cancer require regular evaluation and updating. As this is costly, evidence is needed of demand for this information, past and future potential usage and format preferences. An audit and evaluation of key familial cancer resources was undertaken. The audit used the CGE electronic database that tracks resource dissemination in NSW and interstate. An evaluation was undertaken with the NSW Familial Cancer Services and outreach genetic counseling services, as well as other interstate users. 
28/42 completed the evaluation that sought to determine how the resource was used, preferred format, and desired edits. Findings for all the resources were similar with preference for format being print $(61 \%-69 \%)$ with some wanting both print and electronic formats $(31 \%-33 \%)$. Generally, for those who provided the material to clients $(67 \%-83 \%)$, half used the material in consultations and half sent it home with the patient. The majority requested the information to be updated. Since the audit and evaluation two of the identified key resources addressing preventative surgery to manage cancer risk have undergone expert peer review and consumer testing; Information For Women Considering Preventative Mastectomy Because of a Strong Family History Of Breast Cancer and Risk Management Options For Women at Increased Risk Of Developing Ovarian Cancer. These materials are available in print and electronic format. Consultation is now underway to produce two decision aids on genetic testing for familial cancer risk.

\section{BEYOND CSI: ETHICS IN THE CLASSROOM [80]}

M. Saleh', L. Melton², D. Brewer'², and K. Barlow-Stewart'

The Centre for Genetics Education, NSW Health, Australia

Children's Medical Research Institute, Australia

Given the media attention DNA and genetic science gets, it is important to help teachers sift through issues that are sensationalized to a large audience which includes school aged young adults. A high school science class may be the only time many of our future community decision makers have formal training in the impact genetic technologies have on society. Our aim is to assess how this process can best be facilitated to empower high school science teachers with the confidence and tools to ensure this opportunity is not lost. Since 1997, the Centre for Genetics Education has been providing opportunities for teachers to gain an understanding of the importance of teaching human genetics and its social impact at all levels of science from Years 7 to 12. This has been achieved in the past by providing full-day workshops in a 'train the trainer' lecturestyle learning environment. More recently, in partnership with the Children's Medical Research Institute, the Centre has participated in a comprehensive full day research update that includes practical DNA demonstrations and 'meet the scientists' sessions. Teaming this hands-on and specialized scientific update with a session that brings the research information back to the social and human context has been a useful way of illustrating how ethics and gene technology need to be considered on equal terms. Taking into account the resources needed for undertaking these learning programs and the feedback collected from them, we propose the most effective way in which to maximize teacher attendance and outcomes.

\section{MUTATIONS IN GLYPICAN 6 (GPC6) IMPAIR \\ ENDOCHONDRAL OSSIFICATION AND CAUSE RECESSIVE OMODYSPLASIA [47]}

R. Savarirayan and A.-B. Campox Xavier

Murdoch Children's Research Institute, Melbourne, Victoria, Australia

Autosomal recessive omodysplasia (ARO; MIM \#258315) is a primary skeletal dysplasia characterized by severe short stature, congenital micromelia, and distinct facial dysmorphism. To determine the molecular basis of ARO, we performed a genome-wide linkage analysis in 5 families segregating ARO. A unique locus of linkage was found on chromosome 13 (13q31.1-q32.2), spanning a region of $4.3 \mathrm{cM}$, where haplotype analysis showed homozygosity in affected individuals of the 4 consanguineous families with no common haplotype shared by these families. Genes in this interval potentially involved in skeletal development and morphogenesis were selected as possible candidates. We identified mutations in GPC6, encoding the heparan sulfate proteoglycan glypican (HSPG) 6 , in 5 families and 1 sporadic case of ARO. We show that glypican 6 is highly expressed in the proliferative zone of the murine growth plate, indicating that it is involved in endochondral ossification, and plays a major role in normal long bone growth. We postulate that the homozygous GPC6 mutations in our patients abrogate the function of this HSPG in the growth plate causing altered FGF signaling and morphogen (IHH) gradients, leading to failure of proliferative chondrocyte terminal differentiation and long bone growth retardation.
MECP2 MODULATES TUBULIN ACETYLATION BY BLOCKING TUBULIN DEACETYLASE ACTIVITY OF HDAC6 [109]

A. Saxena', R. Scaife', K. Croft', J. Christodoulou', P. Zhang', J. Beaumont',

G.J Pelka ${ }^{4}$, P. P. L. Tam ${ }^{4}$, H. Leonard ${ }^{5}$, G. Matthijs ${ }^{6}$, M. Kavallaris ${ }^{7}$, and D. Ravine ${ }^{1,2}$ Western Australian Institute for Medical Research, University of Western Australia, Australia

School of Medicine and Pharmacology, University of Western Australia, Australia Western Sydney Genetics Program, Children's Hospital at Westmead, and Discipline of Paediatrics and Child Health, University of Sydney, Australia

Children's Medical Research Institute, Westmead, Australia

Telethon Institute for Child Health Research, Subiaco, Australia

${ }^{6}$ Laboratory for Molecular Diagnostics, Center for Human Genetics, University of Leuven, Herestraat 49, B-3000 Leuven, Belgium

Children's Cancer Institute Australia for Medical Research, Sydney, NSW, Australia

Methyl CpG binding protein 2 (MeCP2) is known to mediate gene expression by several mechanisms within the nucleus. MeCP2 also has a recently recognised cytoplasmic distribution, which is of unknown functional significance. Here, we report that $\mathrm{MeCP} 2$ maintains microtubule stability via the tubulin deacetylation pathway. We show that MeCP2 associates with microtubules in the cytoplasm, as well as on the mitotic spindle and within the midbody remnant. Use of microtubule inhibitors alters both the cytoplasmic and nuclear distributions of MeCP2. Absence of MeCP2 in a MECP2 mutant cell line is associated with microtubule instability together with reduced amounts of acetylated tubulin. RNAi knockdown of MeCP2 in wild type cells causes reduced amounts of acetylated tubulin, whereas MeCP2 over-expression prompts a rise in the level of acetylated tubulin. Functionally, we have identified an interaction between MeCP2 and the tubulin deacetylase histone deacetylase 6 (HDAC6) and shown through in vitro experiments, that MeCP2 inhibits HDAC6-mediated tubulin deacetylation. Our data reveal that MeCP2 functions to preserve tubulin acetylation.

\section{IS ROUTINE SCREENING FOR LYNCH SYNDROME IN ENDOMETRIAL CANCER USING MICROSATELLITE INSTABILITY AND IMMUNOHISTOCHEMICAL TESTS WORTHWHILE? [46]}

L. Schofield ',2, A.-M. Sherwood ${ }^{2}$, F. Grieu², B. Brennan ${ }^{3}$, C. Stewart ${ }^{3}$, J. Goldblatt ${ }^{1,4}$, and B. lacopetta ${ }^{2}$

${ }^{1}$ Genetic Services of Western Australia, King Edward Memorial Hospital, Australia 2 School of Surgery. University of Western Australia, Australia

${ }^{3}$ PathWest, King Edward Memorial Hospita, Australia

${ }^{4}$ School of Paediatrics and Child Health, University of Western Australia, Australia

Women with Lynch Syndrome (LS) have an increased risk of developing endometrial cancer (EC) due to an inherited mutation in one of the mismatch repair (MMR) genes. The detection of suspected cases of LS amongst all EC cases is difficult because there are no well-defined clinical or pathological criteria to indicate the presence of an underlying germline mutation. Despite the diagnosis of approximately 150 cases per year in Western Australia, only a small number are referred to Genetic Services of Western Australia for further ascertainment as possible LS. Almost all cancers that arise in the context of LS demonstrate microsatellite instability (MSI) and concomitant loss of MMR protein expression. The aim of this study was to evaluate whether MSI and loss of MMR expression can be used to identify potential LS cases amongst young EC patients. Archival tumour samples from 250 cases of EC diagnosed in women aged $<60$ years were examined for these molecular features using standard PCR and immunohistochemical (IHC) techniques. A total of $6.8 \%$ $(17 / 250)$ cases showed MSI using the BAT26 mononucleotide marker, of which all but one had complete loss of expression for one or more MMR proteins. The interpretation of IHC results was complicated by frequent heterogeneity of staining, including partial and clonal loss of expression. To date, a germline mutation has been identified in 3 cases with MSI and/or complete loss of expression. The utility of routine molecular testing for the identification of LS amongst EC patients will be discussed.

\section{DISCLOSURE OF GENETIC RESEARCH RESULTS AFTER} DEATH OF PEDIATRIC PATIENTS - IS IT ETHICAL? [15]

\footnotetext{
A. Sexton ${ }^{3}$, M. Sahhar ${ }^{2,3}$, D. R. Thorburn ${ }^{1,2,4}$, and S.A. Metcalfe ${ }^{1,2}$

Murdoch Childrens Research Institute, Royal Children's Hospital, Australia

Department of Paediatrics, The University of Melbourne, Australia

Genetic Health Services Victoria, Royal Children's Hospital, Australia

${ }^{4}$ Victorian Clinical Genetics Services Pathology, Royal Children's Hospital, Parkville, Victoria, Australia
}

The potential for genetic diagnoses through research is increasing in proportion to the rapid rate of gene discovery. Public concern about use of stored tissue or organs from paediatric autopsies in UK hospitals without parents' consent resulted in the 2004 overhaul of the Human Tissue Act. The removal and retention of organs during autopsy caused much distress to parents who were unaware that this had occurred. Whilst this was a 
clear ethical failure to uphold the rights of parents, and led to new legislation, there are several areas in current law and guidelines that remain unclear. Genetic diagnosis of paediatric patients after death occurs for conditions including cardiac deaths, SIDS and mitochondrial conditions. Several recent studies on the impact of delayed genetic diagnosis have found that parents want to be informed, even when the renewed contact and genetic results are unexpected. We conclude that it is critical that parents' wishes are respected at the time that samples are obtained from their child. This may have occurred at an extremely traumatic time in their child's illness. The initial consent process may include one-time consent for on-going research, and any preference to opt out of receiving further research results noted. Clinicians and researchers should formulate plans: (1) to provide families with research updates, (2) for psychological support, (3) for on-going assistance with understanding and adjusting to a delayed genetic diagnosis (Sexton and Metcalfe 2008, JAMA 300: 1693). Such considerations may help minimise the impact of receiving this new genetic information.

\section{HEALTH AND DEVELOPMENT OF CHILDREN WITH DE NOVO APPARENTLY BALANCED CHROMOSOME REARRANGEMENTS DETECTED BEFORE BIRTH [125]}

A. Sherwen', I. Sinnerbrink ${ }^{2}$, D.Amor ${ }^{4}$, E. Kirk², B. Meiser ${ }^{3}$, and J. Halliday'

'Murdoch Children's Research Institute, Australia

2 Sydney Children's Hospital, Australia

${ }^{3}$ University of New South Wales, Australia

${ }^{4}$ Genetic Health Services Victoria, Australia

The health outcome for children diagnosed prenatally with a de novo apparently balanced chromosome rearrangement (ABCR) is unpredictable. The limited research conducted has indicated a small increased risk of abnormality. This research project aimed to determine if children with a de novo ABCR diagnosed prenatally experienced any health, development or behavioral concerns. The effect of the prenatal diagnosis on parents and parent-child attachment, and plans for disclosure of the test result were also examined. Mothers and children between 3 and 12 years, with a prenatally diagnosed de novo ABCR were recruited. Mothers completed a questionnaire and children completed an educational achievement and development assessment. Validated scales measuring health, development and behavior, as well as qualitative questions regarding the disclosure of the test result, were included in the questionnaire. Sixteen questionnaires were available for the preliminary analysis. Descriptive analysis found no differences between the study group and the normative samples used to validate information to their children, often more than 10 years after they received it themselves. The difficulties associated with recruitment have highlighted the need for a multi-centre prospective follow-up study for this type of research.

\section{A NATIONAL POLICY FOR RARE DISORDERS [135] \\ D. Sillence \\ Discipline of Genetic Medicine, University of Sydney, The Children's Hospital at Westmead Clinical School NSW, Australia}

The Garling report into Acute Care Services in NSW Public Hospitals 2008 draws attention to the need for policy to address acute care for rare disorders and uses the Ehlers-Danlos syndromes as an example. The European Organisation for Rare Diseases in its 2005 position statement on the provision of clinical services and Innovative Therapies (Orphan drugs) for rare disorders also uses Ehlers-Danlos syndromes as an example of the delays in diagnosis and lack of resources in its needs-based recommendations for Centres of Expertise with rare disorders. Classic EDS is extremely rare and parents frequently tell their stories of ignorance and outright incompetence of emergency departments in managing their disorders. A rare disorder is one which effect less than 500 patients per million. They are primarily genetic with approximately 6,000 known disorders. The authors of EURORDIS noted the present lack of access to correct diagnosis, information, scientific knowledge, high cost of the few existing drugs and inequities in care. Legislation to recognise rare disorders from a public health perspective and empower development and delivery of centres with expertise in Australia and New Zealand who can advise, act as a resource and evaluate new (orphan) therapies is sorely needed. The legislative program needed will be discussed on the basis of 30 years experience with policy development and advocacy. An Orphan disease policy should include development of policy for delivery of innovative therapies (Orphan Drugs). President Sarkozy recently approved a EURO 140 million program to develop the Rare Disorders Program in France.
CYTOGENETIC AND FISH STUDIES (USING ETV6/RUNX1 PROBE) ON BONE MARROW SAMPLES FROM 78 CHILDREN WITH PRECURSOR B CELL ALL [82]

E. Smith, L. St. Heaps, D. Hung, S. Diaz, P. Sharma, and L. Robson

Department of Cytogenetics, Children's Hospital at Westmead, Westmead, Australia

Precursor B cell acute lymphoblastic leukaemia (ALL) in children is associated with normal or various abnormal karyotypes, varying from favourable to very poor prognosis. While some abnormalities are clearly visible eg hyperdiploidy, the $\mathrm{t}(12 ; 21)(\mathrm{p} 13 \mathrm{q} 22)$ is cryptic. It is important to confirm the presence of this translocation due to its favourable outcome. Over 11 years (1998 to end 2008) FISH has been used to characterise normal, and certain abnormal, karyotypes in children with precursor B cell ALL. FISH has been performed on a total of 76 new patients, aged 1 to 18 years, and two patients diagnosed in childhood, now aged 24 and 25 years, who relapsed after 10-15 years of paediatric cure. The probe used in each case was the extra signal ETV6/RUNX1 (Vysis) on bone marrow cultured cell suspensions, with the primary aim of detecting ETV6/RUNX1 fusion. FISH showed an abnormality in 49 patients $(63 \%)$. Of interest is the variety of abnormalities seen ; 18 with fusion alone, 9 with fusion + deletion ETV6, 6 with deletion ETV6 alone, 8 with RUNX1 amplification alone, 3 with fusion + RUNX1 amplification, one with deletion ETV6 + amplification RUNX1, and 4 with fusion + deletion RUNX1 + amplification of RUNX1. Apart from the cryptic fusion, many of these other FISH abnormalities were not suspected on cytogenetics, indicating that FISH is an integral part of the assessment of children with precursor B cell ALL. Some of the abnormalities above may affect the prognosis, as yet undelineated.

\section{MUTATION SCANNING IN THE BREAST CANCER} SUSCEPTIBILITY GENE PALB2: ARE SOME FEMALE CARRIERS AT HIGH RISK OF BREAST CANCER [152]

M. C. Southey', M.Tischkowitz ${ }^{2,3}$, Z. L. Teo', F. Odefrey', N. Sabbaghian², G. B. Byrnes ${ }^{4}$, I.Winship ${ }^{5}$, Breast Cancer Family Registry, kConFab, M. R. E. McCredie', G. G. Giles ${ }^{7}$, D. E. Goldgar ${ }^{8}$,W. Foulkes'2, and J. L. Hopper ${ }^{9}$ ${ }^{\prime}$ Genetic Epidemiology Laboratory, Department of Pathology, University of Melbourne. Victoria, Australia

2 Program in Cancer Genetics, Departments of Human Genetics and Oncology, McGill University, Montréal, QC, Canada

${ }^{2}$ Segal Cancer Centre, Sir Mortimer B Davis Jewish General Hospital, Montreal, Canada ${ }^{4}$ The International Agency for Research on Cancer, Lyon, France

${ }^{5}$ Department of Medicine, University of Melbourne and Royal Melbourne Hospital, Australia ${ }^{6}$ The University of Otago, Dunedin, New Zealand

Cancer Epidemiology Centre, The Cancer Council Victoria, Australia

${ }^{8}$ University of Utah School of Medicine, Salt Lake City, United States of America

${ }^{9}$ Centre for Molecular, Environmental, Genetic and Analytic Epidemiology, The University of Melbourne, Victoria, Australia

Deleterious mutations in PALB2 have been reported to be associated with, on average, a two to three fold increased risk of breast cancer on top of any increased risk associated with a family history of breast cancer. Consequently, the women who have germline mutations in PALB2 who also have a strong family history of breast cancer are predicted to be at a high absolute risk of the disease comparable to that for women who carry germline mutations in BRCA1 or BRCA2. We are conducting a large case-control-family study using population-based and clinic-based resources from Australia, USA and Canada. We have scanned PALB2 using high-resolution melt (HRM) curve analysis. The DNA samples that have aberrant melt profiles are sequenced to specifically identify the genetic variants. Missense variants are assessed for possible significance using a variety of in-silico methods such as SIFT, PolyPhen and GVGD. We have identified a series of genetic variants in PALB2 ranging from common to rare and including nonsense mutations. The results of our study will be used to estimate risk associated with carrying a PALB2 mutation as a function of family history. The estimation of the significance of the variants we identify, and how this is incorporated into the analysis is fundamentally important to the outcome. It is possible that some of these women are at high risk, comparable to the risk with BRCA1 or BRCA2 mutations. This research will provide definitive information about PALB2 and may impact on clinical genetics services worldwide.

\section{TWO DIFFERENT OUTCOMES FOR CLIENTS WITH SIMILAR INTERSTITIAL DELETIONS ON THEIR X-CHROMOSOME [207]} M. Sproule

Genetic Health Services Victoria, Australia

Two different women were seeking genetic counselling due to a diagnosis of an interstitial deletion on their X chromosome. Even though they both had similar genotypes, their phenotypes were markedly different. One client was referred to the service from her Gynaecologist, due to irregular periods and short stature, but an otherwise seemingly normal development. The other client was referred to the service due to retinitis pigmentosa; 
oral ulcers; and elevated ammonia, glutamine, and urinary orotic acids levels, but normal height. Both clients wanted to have children and were keen to know how their karyotype could impact on their ability to have children. We will discuss how their similar deletion resulted in a different phenotype, what further testing/investigations were arranged, and how the diagnosis affected them.

\section{THE GENETIC BASIS FOR ADMISSIONS TO A PAEDIATRIC HOSPITAL [141]}

\author{
C. Stanley ${ }^{1,2}$, A. Bankier ${ }^{3}$, C. Rose ${ }^{3}$, K. Robinson', and J. Halliday ${ }^{2}$ \\ Faculty of Health Sciences, La Trobe University, Australia \\ Murdoch Childrens Research Institute, Parkville, Australia \\ Genetic Health Services, Victoria, Australia
}

This research describes the magnitude of genetic disorders at a Victorian paediatric hospital in 2007, and compares results from two earlier studies done in 1985 and 1995 . The medical records of 1,313 admitted patients in a two week period were examined and each admission assigned to one of 9 categories. Categories one to four comprise simple genetic disorders, five is multifactorial and the other four represent developmental conditions and illnesses with and without genetic components. Use of OMIM and POSSUM, and consultation with experts in clinical genetics helped to determine 'genetic load'. In 2007, the proportion of admitted episodes for single gene and chromosomal disorders was $9.8 \%$, multifactorial was $10.7 \%$, and the overall proportion with a genetic component was $48.9 \%$, a significant increase from 1995 to $2007\left(\chi^{2}=32.31, p<.01 ; \mathrm{RR}=1.31, \mathrm{Cl}\right.$ $1.19-1.44)$. Much of this increase was in admissions in category 8 (illness with genetic component) which rose from $15.6 \%$ in 1995 to $25.8 \%$ $\left(\chi^{2}=34.53, p<.01\right)$. This was somewhat counterbalanced by a decrease in autosomal recessive disorders from $9.4 \%$ in 1985 to $4.4 \%$ in 2007 . Patients with single gene and chromosomal disorders had a higher mean number of previous admissions and lengths of stay, thus supporting the findings of previous studies that these patients as a group place a higher demand on hospital resources than non-genetic patients. Knowledge of the magnitude and details of genetic disease and related hospital data are important for health care service planning and for monitoring the effects of new genetic technologies and services.

\section{DE NOVO DELETION OF THE ATTRACTIN-LIKE 1 (ATRNL1)} GENE RESULTING IN A DISTINCT PHENOTYPE [84]

\section{Z. Stark ${ }^{1,2}$, D. Bruno ${ }^{1,2}$, and D.Amor ${ }^{1,2}$ \\ Genetic Health Services Victoria, Australia \\ Murdoch Childrens Research Institute, Melbourne, Australia}

Attractin (ATRN) and its homologue attractin-like 1 (ATRNL1) are genes that are highly conserved among species, and encode a single-pass transmembrane glycoprotein that is postulated to be involved in cell adhesion and signalling events. Atrnll and atrn have been well characterized in mice but there is a lack of information about their roles in human development. Attractin is widely expressed in mouse brain, skin, heart, kidney, liver and lung. Atrn null mice exhibit a severe neurological phenotype comprising tremor, hypomyelination and spongiform degeneration, in addition to altered fur colour, reduced body weight and adiposity, whereas atrn heterozygotes and atrnl1 null mice are grossly normal. Overexpression of atrnll compensates for loss of atrn in the skin and brain, suggesting that there is considerable overlap between their functions. We provide the first description of a human patient with a heterozygous deletion of ATRNL1. The patient presented with a novel and distinctive phenotype comprising dysmorphic facial appearance, ventricular septal defect, toe syndactyly, radioulnar synostosis, postnatal growth retardation, moderate global developmental delay and ataxia. A $325 \mathrm{~kb}$ de novo deletion in ATRNL1 was demonstrated using SNP microarray and confirmed using BAC probes. We postulate that our patient's features are caused by the deletion of ATRNL1, and provide further insight into the biological role of ATRNL1 in human development.

\section{THE IMPLEMENTATION OF NON-INVASIVE PRENATAL TESTING FOR DOWN SYNDROME [118]}

\section{Susman', D. Amor ',2, E. Muggli', A. Jaques', and J. Halliday' \\ Murdoch Childrens Research Institute \\ Genetic Health Services Victoria, Australia}

The discovery of free fetal nucleic acids in maternal serum has led to the development of new tests for noninvasive prenatal detection of Down syndrome that are predicted to revolutionize the practice of prenatal screening and diagnosis. There are currently two competing technologies, one relying on differences between maternal and fetal alleles and the presence of genetic polymorphisms, and the other utilizing direct shotgun sequencing. In addition to the effect on pregnant women, the implementation of diagnostic non-invasive prenatal testing will also have major implications for cytogeneticists, serum screening programmes, genetic counsellors, obstetric ultrasonographers, and policy makers. Utilizing the comprehen- sive prenatal screening and testing data that have been collected in Victoria for many years, we have generated models to predict the impact of noninvasive prenatal testing in our population. Using this approach, we show that the implementation of non-invasive prenatal testing may increase the number of Down syndrome cases detected by $23 \%$, however, there would be a corresponding decrease by $60 \%$ in the overall detection of chromosome abnormalities. Our most extreme model also predicts the number of prenatal diagnostic procedures (CVS and amniocentesis) could decrease by a substantial $90 \%$ in our population, from the current $4500 /$ year to less than 500/year. Non-invasive prenatal testing for Down syndrome will result in a trade-off between significantly fewer invasive procedures and greater detection of Down syndrome, and decreased detection of other types of chromosome abnormalities.

\section{REPORT FROM THE EUROPEAN MOLECULAR QUALITY NETWORK BEST PRACTICE GUIDELINES MEETING FOR DUCHENNE MUSCULAR DYSTROPHY GENETIC TESTING, NOVEMBER 14-16, 2008 [137]}

P.J.Taylor

SEALS Genetics Laboratories, Prince of Wales Hospital, Randwick NSW, Australia

In view of recent developments in molecular testing techniques and therapeutic strategies, the aim of this meeting was to prepare updated guidelines for molecular testing of the dystrophinopathies. The primary focus was on harmonising diagnostic testing procedures, but the recommendations also included guidelines for interpretation, mutation nomenclature and report writing. The main recommendations were that the dystrophin gene mutation analysis should screen for both copy number mutations and small sequence mutations. Initially, a PCR based test for copy number mutations (whole exon deletions and duplications) that tested all 79 exons of the dystrophin gene should be performed for samples referred for suspected dystrophinopathies such as Becker and Duchenne muscular dystyrophies. MLPA was generally considered to be currently the most appropriate and cost-effective method but, in light of rapid progress in the development of other methods for the assessment of gene copy number (including array $\mathrm{CGH}$ ), no specific methodology was recommended. If gene copy number analysis did not identify a mutation, and there was additional clinical and laboratory evidence supporting the diagnosis of a dystrophinopathy, DNA sequencing of the coding regions of the dystrophin gene from genomic DNA (or cDNA sequencing from mRNA) should be considered best practice. This was considered to be important in light of developing therapies for which the knowledge of the specific mutation or mutational class is reliant. It was acknowledged, however, that many laboratories, especially those in developing countries, would not be able to offer this level of service due to the high costs currently associated with this testing.

\section{EXTRAPYRAMIDAL SYMPTOMS AND MEDICATION USE IN} MUCOPOLYSACCHARIDOSIS TYPE III [8]

\section{M.Tchan and D. O. Sillence}

Discipline of Genetic Medicine, The University of Sydney, The Children's Hospital at Westmead Clinical School NSW, Australia

We report the case of a 16 year old male with Mucopolysaccharidosis III type A (Sanfilippo syndrome) who was commenced on risperidone for behavior management. He rapidly developed extrapyramidal symptoms that have not resolved. The medication histories of twenty patients with Mucopolysaccharidosis III seen at a Lysosomal Storage Diseases Clinic were reviewed to determine the incidence of extrapyramidal side effects. Six patients had been treated with risperidone, olanzapine or lamotrigine. Five of these patients developed extrapyramidal side effects. The incidence of extrapyramidal side effects was considerably higher than expected. We suggest that these medications be used with considerable caution in these patients.

\section{AN ETHICAL DILEMMA: ETHICS APPROVAL FOR RESEARCH ON INTELLECTUAL AND DEVELOPMENTAL DISABILITIES [56]}

\section{A.Thomson', E.J. Glasson',2, P. Roberts', and A. H. Bittles'}

${ }^{1}$ Edith Cowan University, Perth, Australia

University of Western Australia, Perth, Australia

Intellectual and developmental disabilities (IDD) are a global health concern. However, ethical issues concerning people with IDD are currently complex, with approval for research involving family carers and requiring access to linked data on affected persons especially problematic. In Australia, applications to multiple Human Research Ethics Committees (HRECs) have to be submitted in sequence, with amendments requested by one committee requiring ratification by all others. Informed and proxy consent issues also arise, with privacy concerns prohibiting researchers from direct contact with potential participants and their families, which necessitates invitation letters issued via service agencies. Many adults 
with IDD are incapable of self-consent and have no legal guardian, and carers may fail to respond to these communications. Under both circumstances there is significant selection bias, with under-representation of persons with poorer functionality. This difficult situation could be alleviated by adoption of the National Ethics Application Form (NHMRC (2007), which would allow multiple applications in a single format, substantially reduce preparation time, help to provide consistent and complete information to HRECs, and assist multi-centre research. Direct meetings between researchers and HRECs would also be beneficial, and consultation between HRECs prior to their responses to researchers would be constructive. Although potentially time-consuming and incurring some expense, applications to the relevant State Authority for the award of limited guardianship could significantly facilitate proxy consent. Collectively, these measures should assist in the recruitment of people with IDD as research participants, to their benefit and that of their families and carers.

\section{NEXT-GENERATION SEQUENCING FOR IDENTIFICATION OF NOVEL GENES CAUSING COMPLEX I DEFICIENCY [88]}

E. Tucker', A. G. Compton', S. E. Calvo ${ }^{2,3}$, D. J. Pagliarini', V. K. Mootha², and D. R.Thorburn'

Murdoch Childrens Research Institute, Royal Children's Hospital \& Department of Paediatrics, University of Melbourne, Australia

${ }^{2}$ Center for Human Genetic Research, Massachusetts General Hospital, Boston \& Department of Systems Biology, Harvard Medical School, Boston \& Broad Institute of MIT and Harvard, Cambridge, United States of America

${ }^{3}$ Harvard-MIT Division of Health Sciences and Technology, Cambridge, United States of America

Humans generate energy via oxidative phosphorylation, which occurs on the inner membrane of mitochondria. An important enzyme involved in this process is Complex I, which is comprised of 45 subunits, seven encoded by mtDNA. Complex I deficiency is the most common cause of mitochondrial disease. Human Complex I deficiency can be caused by mutations in genes encoding all seven mtDNA subunits, 12 nuclearencoded subunits and 5 other proteins required for assembly of Complex I into a functional enzyme complex. Despite knowledge of more than 50 genes encoding Complex I subunits or assembly factors, about half of all patients fail to have a causative mutation identified. Presumably, many other genes involved in Complex I function and assembly await discovery. Putative Complex I-related genes have been identified by functional studies in cell lines or species other than humans, and by phylogenetic profiling. We have performed next generation sequencing of $>80$ putative Complex I-related genes in a cohort of 103 patients with Complex I deficiency, 59 of whom have no genetic diagnosis. Correction studies will be performed to confirm the pathogenicity of mutations. This project aims to provide diagnoses for patients with Complex I deficiency and to identify new Complex I-related genes.

\section{FROM RESEARCH TO DIAGNOSTICS : RUNX1 MUTATION ANALYSIS IN LEUKEMIA [140]}

C.Vakulin', G. McKavanagh', X. Li', C. Carmichael' ${ }^{2}$ E.Wilkins ${ }^{2}$, L. Lipton ${ }^{7}$ B. Mercorella', M.Altree ${ }^{4}$, N. Patton ${ }^{5}$, J. Cooney ${ }^{6}$, R. D'Andrea ${ }^{5}$, G. Suthers ${ }^{4}$, L. Rawlings', J. Carroll', and H. Scott'

${ }^{\prime}$ Institute of Medical and Veterinary Science, Adelaide, SA, Australia

${ }^{2}$ Molecular Medicine, WEHI, VIC, Australia

${ }^{3}$ Flinders Medical Centre, SA, Australia

‘ Womens' and Childrens' Hospital, SA Familial Cancer Unit, SA, Australia

Royal Adelaide Hospital, Haematology Unit, SA, Australia

${ }^{6}$ Clinical Haematology, Royal Perth Hospital, WA, Australia

7 University of Melbourne, Royal Melbourne and Western Hospitals, Australia

Since 2004, the Australian Familial Haematological Cancer Study has identified Australian kindreds with familial predisposition to haematological malignancies. To date, germline mutations in the RUNX1 gene have been identified in 2 Australian families with a familial platelet disorder and a predisposition to acute myelogenous leukaemia (AML). In family 1, a novel germline heterozygous nonsense mutation (c.958C $>$ T (p.R320X)) was found in exon 7 of the RUNX1 gene. In family 2 a deletion of exons 2,3 and 4 of the RUNX1 gene was detected by MLPA and confirmed by long PCR. Having confirmed these mutations in a diagnostic setting, we are now offering a comprehensive RUNX1 mutation screen including complete gene sequencing in both sporadic and familial leukemia.
USING GENOTYPE TO TAILOR PRESCRIBING OF NICOTINE REPLACEMENT THERAPY: A RANDOMISED CONTROLLED TRIAL ASSESSING IMPACT UPON ADHERENCE [155]

S.Whitwell', C. Hill', A. J.Wright', A.T. Prevost ${ }^{2}$, M. R. Munafò ${ }^{3}$, P.Aveyard ${ }^{4}$,

D. Armstrong', A. Louise Kinmonth' ${ }^{2}$, S. Sutton ${ }^{2}$, and T. M. Marteau

'King's College London, United Kingdom

2 University of Cambridge, United Kingdom

${ }^{3}$ University of Bristol, United Kingdom

${ }^{4}$ University of Birmingham, United Kingdom

Pharmacogenetics has the potential to increase the effectiveness of medicines in two key ways: first, it can enable treatment to be tailored more closely to individual biology; second, it can increase expectations of treatment effectiveness and hence motivation to take the treatment. This trial is designed to evaluate the latter route in the context of prescribing Nicotine Replacement Therapy (NRT) for smokers seeking help with smoking cessation. ISRCTN: 14352545. An open label, parallel groups randomised trial in which smokers using stop smoking services in primary care are randomly allocated to one of two groups: (1) NRT top-up prescribing tailored by DNA analysis (OPRM1 gene) (genotype), or (2) NRT top-up prescribing tailored by heaviness of smoking (phenotype). The primary outcome was the proportion of NRT prescribed (in milligrams) that is consumed each day in the first four weeks of quitting. Collecting the primary outcome on 630 smokers allows sufficient power to detect a $7.5 \%$ difference in the mean proportion of NRT consumed using a 2-tailed test at the $5 \%$ level of significance. Recruitment of 633 smokers was completed in August 2008. The analysis will be completed in March 2009.

\section{INTEREST IN PREDICTIVE GENETIC TESTING FOR SUSCEPTIBILITY TO MAJOR DEPRESSION IN A LARGE NON-CLINICAL POPULATION [9]}

A.Wilde', B. Meiser', P. B. Mitchell ${ }^{1,3}$, and P. R. Schofield ${ }^{4,5}$

'School of Psychiatry, University of New South Wales, Australia

${ }^{2}$ Prince of Wales Clinical School, University of New South Wales, Australia

${ }^{3}$ Black Dog Institute, Australia

${ }^{4}$ Prince of Wales Medical Research Institute, Australia

'School of Medical Sciences, University of New South Wales, Sydney, Australia

A large number of studies have demonstrated an association between a functional polymorphism in the promoter region $(5-H T T L P R)$ of the serotonin transporter gene (SCL6A4) and exposure to stressful life events in increasing the likelihood of major depression. Predictive genetic testing presents an opportunity to identify high-risk groups and reduce the burden of depression through intervention strategies at a pre-symptomatic stage. The aims of this study are to evaluate public interest in predictive genetic testing for depression risk genotypes, including tests marketed direct-toconsumer (DTC); and assess public perception of impact on stigma. Quantitative national survey of 1046 participants from a nonclinical population recruited through random digit dialling. The majority of participants were interested in having a predictive genetic test for susceptibility to depression. Interest in having such a test through a doctor was significantly greater than interest in accessing the test DTC via the Internet $(p<$ $.001)$. Self-estimation of being at higher than average risk for depression; endorsement of a genetic model for depression; and having a personal history of mental illness significantly predicted interest in having such a test (all $\mathrm{p}<0.001$ ). Perceived genetic discrimination and privacy issues were not significantly associated with interest in having the test. A significant majority of participants believed evidence of a genetic component for depression would increase rather than decrease stigma associated with this disorder $(p<.001)$. The study highlights the importance of public education about psychiatric genetics, gene-environment interactions, incomplete penetrance of susceptibility genes; and development of appropriate intervention in association with future predictive testing in psychiatry.

\section{SPLICE INTERVENTION TO TREAT DUCHENNE MUSCULAR} DYSTROPHY AND BEYOND [145]

S. Wilton, A. Adams, P. Meloni, R. Johnsen, S. Forrest, K. Greer, L. Stone, C. Mitrpant, and S. Fletcher

Molecular Genetic Therapy Group, CNND, University of Western Australia, Perth, Australia

Clinical trials are underway to demonstrate that antisense oligomers (AOs) can redirect dystrophin gene transcript splicing to excise selected exons, and thereby remove protein truncating mutations that would otherwise lead to Duchenne muscular dystrophy (DMD). Due to the widespread and complex nature of dystrophin gene expression, DMD is a great challenge to any therapy. However, characterization of the gene structure in Becker MD patients presenting with mild phenotypes, indicate that substantial portions of the dystrophin gene can be lost with relatively minor consequences, and some in-frame deletions may only be identified late in life. It as been confirmed that over half of the 79 exons are redundant, when lost in particular combinations. While 10-12 AOs should restore the reading- 
frame in the more common genomic deletion hotspots, scores of AOs will be needed to by-pass the many different protein-truncating mutations spread across the gene. The immediate challenges are (i) to establish effective dosage regimens, (ii) gain acceptance for these personalized genetic medicines as class-specific compounds, (iii) extend the treatment to all potentially amenable mutations in the dystrophin gene and (iv) apply this platform to other acquired and genetic conditions.

\section{IRONXS: ASSESSING THE ACCEPTIBILITY AND FEASIBILITY OF GENETIC SCREENING FOR HAEMOCHROMATOSIS IN VICTORIAN HIGH SCHOOLS [198]}

M.Wolthuizen,' S. Metcalfe, ${ }^{1,2}$ V. Collins, ' I. Macciocca, ${ }^{1,3}$ M.A.Aitken,' L. Bond, ${ }^{1,2}$

K.Allen, ${ }^{1,2,4}$ E. Varley, ' J. Craven,' 'P. Lockhart,' ' G.Wilson', and M. Delatycki',2,3

Murdoch Childrens Research Institute, Australia

Dept Paediatrics, The University of Melbourne, Australia

Genetic Health Services Victoria, Australia

'Royal Children's Hospital, Melbourne, Australia

Population genetic screening for hereditary haemochromatosis, a preventable iron overload condition, warrants reconsideration. ${ }^{1}$ The HaemScreen workplace-based study ${ }^{2}$ found high uptake of screening $(>90 \%)$ for people attending the information session, but only $6 \%$ of eligible participants attended. As an alternative strategy for offering screening we report data from ironXS, a screening program for the C282Y HFE mutation in high schools. Over two years a DVD-based information session was presented to 9187 year 10 and year 11 students at 32 schools (some schools participated both years). Parent consent forms were returned by $50 \%$ of students, with $90 \%$ indicating consent for screening. 3484 students were tested $(42 \%$ overall uptake, males $39 \%$; mean age $=15.6 \mathrm{yrs}$ ). This identified $19 \mathrm{C} 282 \mathrm{Y}$ homozygotes (1 in 183), who attended genetic counselling with their parents, and $375 \mathrm{C} 282 \mathrm{Y}$ heterozygotes ( 1 in 9), who received their result by mail. Knowledge questions at baseline (Q1) were answered correctly by over $90 \%$ of students. A second questionnaire (Q2) was sent one month after results to all homozygotes and heterozygotes, plus a sample of wildtypes (72\% response rate). Knowledge retention was generally very high Mean $( \pm$ SE) scores in homozygote group for STAI at Q1 was $32.2 \pm 2.2$ and $30.0 \pm 2.0$ at Q2 and for SF36 health perception at Q1 was $72.9 \pm 1.5$ and $75.1 \pm 2.5$ at $\mathrm{Q} 2$. These scores reflect no significant change $(P>.05)$ Follow-up includes a questionnaire at 12 months and interviews with students, parents and teachers. We aim to screen 9000 students in total.

${ }^{1}$ Allen et al. NEJM 2008, ${ }^{2}$ Delatycki et al. Lancet 2005

AN IMPROVED METHOD FOR THE DETECTION OF CGG TRINUCLEOTIDE REPEATS IN FRAGILE X SYNDROME [113]

\section{R. Woods and V. Hyland}

Molecular genetics, Haematology, Pathology, Queensland, Australia

Fragile X syndrome (FXS) is the most common known cause of inherited intellectual disability. FXS occurs in both males and females with females having milder symptoms. Fragile $\mathrm{X}$ syndrome is caused by mutations within the Fragile X mental retardation 1 protein gene, FMR1. The most common mutation is due to an increased size of a CGG trinucleotide repeat in the 5 untranslated region of FMR1. Detection of this expansion is usually by two means: PCR and southern analysis. PCR analysis employs using two primers flanking the CGG repeat region and then analysing the fragments generated using capillary gel electrophoresis and ABI GeneMapper V4.0 software. PCR fragments are represented as peaks on a graph which characteristically form stutters from two to 15 stutters, depending on repeat number. Here we describe a modification of the standard PCR method, which utilises the usual flanking primers as well as a 'stutter' primer in the reaction. This modification allows the visualisation of individual CGG trinucleotide repeat peaks as well as the presence of any large expansions which are outside the range of conventional PCR. We have also used this data to generate GeneMapper sizing Bins, thus improving the accuracy of allele size calling. This modification to the method for the detection and sizing of CGG trinucleotide repeats in the FMR1 gene has reduced the risk of missing expansions that may be present within a sample, especially when the amount of material is low as well as providing an improvement in the quality of service.

\footnotetext{
A NOVEL AUTOSOMAL RECESSIVE SYNDROME REMINISCENT OF OMENN SYNDROME [23]

A.Yeung', D. Coman', Dean Tey' ${ }^{2}$, S. Choo' ${ }^{2}$, M. Bahlo ${ }^{3}$, and D. Amor ${ }^{1,4}$

Genetic Health Services Victoria, Murdoch Children's Research Institute, Australia

Department of Immunology, Royal Children's Hospital, Australia

Walter and Eliza Hall Institute of Medical Research, Australia

Department of Paediatrics, University of Melbourne, Australia
}

We report a child of consanguineous parents who presents with features typical of Omenn syndrome (MIM \#603544), an autosomal recessive severe combined immunodeficiency (SCID), characterised by exfoliative dermatitis, failure to thrive, lymphadenopathy, hepatosplenomegaly and chronic diarrhoea. A sibling had died previously with similar manifestations. The mechanism for Omenn syndrome is a defect in the genes RAG1, $R A G 2$ or ARTEMIS responsible for VDJ recombination and the diversification of immunoglobulins and T-cell receptors. Despite the presence of clinical findings and haematological markers typical of Omenn syndrome, including increased IgE and eosinophilia, our patient displayed normal numbers of peripheral B cells, polyclonal T cells and NK cells. This immunophenotype is inconsistent with Omenn syndrome, which is characterised by absent circulating B cells and oligoclonal T cells. Differential diagnoses including primary hypereosinophilia, atypical Di George syndrome, SCID with maternal engraftment, and Histiocytosis X were excluded by laboratory and histological testing. This prompted us to suspect a novel syndrome with autosomal recessive inheritance. In support of this, our patient showed a lack of linkage to regions containing the $R A G 1, R A G 2$ and ARTEMIS genes. We also report the findings of a homology search for candidate genes in chromosome regions shown to be autozygous by descent in our patient.

\section{AN IMPROVED METHOD FOR MEASURING HUNTINGTON} DISEASE CAG TRINUCLEOTIDE REPEAT SIZES [114]

\section{B. Young and V. Hyland}

Molecular Genetics, Haematology, Pathology Queensland, Australia

Huntington chorea is an autosomal dominant neurological genetic disorder. The disease is associated with an increase in the length of the CAG (poly Glutamine coding) triplet repeat in exon one of the Huntington gene. In our laboratory the repeat length is determined by PCR amplification using two independent primer pairs, the forward primers being fluorescently labelled. One primer pair is used for sizing the CAG repeat only, while a second flanking primer pair, amplify both the $\mathrm{CAG}$ and adjacent $\mathrm{CCG}$ repeats. The PCR products are separated electrophoretically and detected using an ABI 3130 Genetic Analyser, then analysed using the GeneMapper 4.0 software. PCR fragments are represented as peaks on a graph and characteristically form stutters of two to twelve peaks, the number of stutters increasing with repeat number. Repeat sizes are called automatically by GeneMapper when peaks fall within sizing Bins. We have generated sizing Bins by PCR amplification using primers designed to create long extended three base stutters. This improvement in the method allows us to automate the sizing procedure. We also use the stutter primers, flanking primers and a set of mutation specific primers for analysis when only a single allele is detected using our routine primers. This improvement allows the detection and sizing of alleles which may not have been detectable due to changes in the primer binding sites. This revised procedure, for the analysis of Huntington gene CAG repeats, reduces the risk of misdiagnosis and improves the quality of the service we provide.

\section{STAKEHOLDER ENGAGEMENT AS AN INTEGRAL PART OF BIOBANKS POLICY DEVELOPMENT [180]}

\section{Youngs, H. Dawkins, C. Molster, and P. O'Leary}

Office of Population Health Genomics, Department of Health, Western Australia, Australia

Following the achievements of the Human Genome Project the role of biobanks in genetic research has increased dramatically and the development of specific governance policies and frameworks has not progressed as rapidly. To address the need for policy around biobanks in Western Australia (WA) the Office of Population Health Genomics initiated a series of stakeholder consultations starting with professional and scientific groups. Initially a wide range of professional and scientific stakeholders formed two committees: an inventory working group (survey of researchers to develop an inventory of biobanks); a discussion paper advisory group (provide expert advice on the biobanks policy). To include an accurate representation of community attitudes to biobanks two deliberative engagement forums were run. Both forums were specifically designed to obtain the informed opinions of a wide range of representatives in the WA community. Randomly selected members of genetic support groups were invited to participate in the first forum and randomly selected members of the WA public were invited to participate in the second. Outcomes from both engagement forums and the stakeholder consultation are key components in the biobanks policy currently being developed for WA. 
TELEHEALTH CANCER GENETIC COUNSELLING: AN EVALUATION OF VIDEOCONFERENCING TO OUTREACH AREAS [63]

E. Zilliacus', B. Meiser', E. Lobb ${ }^{3}$, K. Barlow-Stewart ${ }^{4}$, J. Kirk', A. D. Spigelman ${ }^{6}$, L. Warwick ${ }^{7}$, and K. Tucker ${ }^{8}$

${ }^{\prime}$ School of Psychiatry, University of New South Wales, Australia

${ }^{2}$ Department of Medical Oncology, Prince of Wales Hospital, Randwick, Australia

${ }^{3}$ Calvary Health Care Sydney, Kogarah, Australia

${ }^{4}$ Centre for Genetic Education, Royal North Shore Hospital, St Leonards, Australia

${ }^{5}$ Familial Cancer Service, Westmead Hospital, Westmead, Australia

${ }^{6}$ Hunter Family Cancer Service, Hunter New England Health Service, Newcastle, UNSW St Vincents Clinical School, Australic

${ }^{7}$ ACT Genetic Service, Canberra Hospital, Woden, ACT

${ }^{8}$ Department of Medical Oncology, Prince of Wales Hospital, Randwick, NSW, Australia

Tele-health is the current method of delivering familial cancer services to rural and outreach areas in NSW and ACT, however there has been little research evaluating this form of service delivery. This study aimed to evaluate genetic consultations delivered via telehealth. Fifty-seven women from a proposed sample of 120 from high risk breast/ovarian cancer families were recruited prior to their tele-health session. A self-report questionnaire was administered prior to tele-health and one month post-consultation. A significant increase in cancer genetics knowledge $(p=.001)$ and perceived personal control $(p=.005)$ was reported post-tele-health. Anxiety and depression were significantly reduced. Participants reported a high level of satisfaction with the technology. These preliminary results indicate that telehealth cancer genetic counselling is effective. Updated results of this ongoing study will be reported and discussed.
DETECTION AND QUANTIFICATION OF CIRCULATING CELLS IN PATIENTS WITH CUTANEOUS MALIGNANT MELANOMA [168]

M. Ziman', M. Millward'2, R. Pearce', M. Brown', S. Medic', R. Slattery', M. Lee²,

S. Lazic ${ }^{3}$, P. Kumarasanghe ${ }^{2}$, J. Cole' ${ }^{2}$, and P. Heenan ${ }^{2}$

${ }^{\prime}$ Edith Cowan University, Australia

${ }^{2}$ University of Western Australia, Perth, Australia

${ }^{3}$ University of Cambridge, United Kingdom

To detect, characterise and quantify circulating melanoma cells in patients with cutaneous malignant melanoma. This research will assist with development of a blood test for the detection of melanoma micrometastases in patients before and after surgery. End point RT-PCR and Realtime quantitative qRT-PCR were used to assess the presence of circulating melanoma cells in peripheral blood. Several molecular markers were used to test 200 melanoma patients and 70 healthy volunteers. The frequency and level of expression of markers was correlated to Breslow tumour thickness and tumour progression and results were statistically analysed. Antibodies to melanoma cell markers were used in flow experiments to isolate and quantify circulating cells from patient blood samples. Control blood samples spiked with cells from metastatic melanoma cell lines were used as positive controls. Markers of circulating melanoma cells were detected in peripheral blood of $79 \%$ of melanoma patients and in only $11 \%$ of healthy volunteers. Several markers showed a higher detection rate overall, regardless of tumour thickness, whereas some markers showed higher levels in patients with thicker tumours $(p<.001)$. Assay sensitivity tests showed that markers could be detected from as few as 5 cells per blood sample. Notably migrating melanoma cells were found in peripheral blood of patients with early stage tumours and in patients from whom tumours were removed several years previously. Flow cytometry experiments confirmed these results. The molecular markers utilised here show the presence of circulating melanoma cells in peripheral blood of patients. Further research is required to identify the molecular signature of metastatic circulating melanoma cells. 